\title{
A differential equation with a state-dependent queueing delay
}

\author{
István Balázs, Tibor Krisztin ${ }^{\dagger}$
}

\begin{abstract}
We consider a differential equation with a state-dependent delay motivated by a queueing process. The time delay is determined by an algebraic equation involving the length of the queue for which a discontinuous differential equation holds. The new type of state-dependent delay raises some problems that are studied in this paper. We formulate an appropriate framework to handle the system, and show that the solutions define a Lipschitz continuous semiflow in the phase space. The second main result guarantees the existence of slowly oscillating periodic solutions.
\end{abstract}

Keywords: state-dependent delay, queueing delay, differential inclusion, return map, slow oscillation, fixed point, ejectivity

AMS subject classifications: $34 \mathrm{~K} 05,34 \mathrm{~K} 09,34 \mathrm{~K} 13,34 \mathrm{~K} 32$

\section{Introduction}

We consider a system which is composed of a delay differential equation and auxiliary equations defining the delay. The delay differential equation satisfies a negative feedback condition analogously to earlier works by Mallet-Paret and Nussbaum [20, 21], Arino, Hadeler, Hbid and Magal [2, 19], Krisztin and Arino [14], Walther [31, 33, 30, 29]. In [20, $29]$ the state-dependent delay was an explicitly given function (i.e., no auxiliary equation). Walther $[31,30]$ studied problems where the state-dependent delay was defined by an algebraic relation, and in a suitable phase space it was possible to eliminate this auxiliary equation. Arino, Hadeler, Hbid, Magal [2, 19] and $\mathrm{Hu}, \mathrm{Wu}[11]$ considered an equation where the auxiliary equation for the delay was given by an ordinary differential equation. Here we study a differential equation with a state-dependent delay where the delay is defined by two auxiliary equations: an algebraic equation and a differential equation with a discontinuous right hand side. The considered system is interesting from the theoretical point of view since previous results do not seem applicable here. On the other hand, the system is a prototype of rate control problems with delays appearing naturally in queueing processes.

The particular model, that motivated our study, was introduced by Ranjan, La and Abed in $[26,25]$. The problem is specified for a simple computer network, however,

*MTA-SZTE Analysis and Stochastics Research Group, Bolyai Institute, University of Szeged, Szeged, Aradi v. tere 1, 6720, Hungary.

†Bolyai Institute, University of Szeged, Szeged, Aradi v. tere 1, 6720, Hungary. 
analogous models appear, e.g., in more general computer networks, in road networks, in biological networks, or in general in those processes where a bottleneck phenomenon slows down the performance or capacity of a system, see, e.g., [27, 5, 8]. The model is a fluid model, although data are usually discrete. When we talk about data transfer, we can think of a liquid flowing in a pipe. In the derivation of the model sometimes it is helpful to use a discrete version of the phenomenon and to mention units of data. In these cases the continuous model equations are obtained by taking limits. We hope this does not cause confusion. So, consider a network containing a single user and a single server. The user's transmission rate satisfies the bound $0<a \leq x(t) \leq b$, where $b$ is a user-specific physical limitation, and the lower bound $a$ is due to the fact that the user needs to probe the congestion level of the network by continuously transmitting data. The server processes the incoming data by the capacity $c \in(a, b)$. Kelly [12] introduced the utility $U(x)$ and the price $p(x)$ per unit flow of the processing, when the rate is $x$. Under natural conditions on the functions $U(\cdot)$ and $p(\cdot)$, there is an optimal rate $x_{*} \in(a, c)$ (balancing between the utility and the price of processing) as the unique maximum of the expression $U(x)-\int_{0}^{x} p(y) d y$ subject to the constraint $0<x \leq c$, see Kelly et al. [13]. In addition, [13] proposed an end user rate control algorithm as the differential equation

$$
\dot{x}(t)=\kappa\left[x(t) U^{\prime}(x(t))-x(t) p(x(t))\right]
$$

where $x U^{\prime}(x)$ is the price per unit time the user is willing to pay for the processing, $x p(x)$ is the price charged by the server, $\kappa>0$ is a gain parameter. The solutions of equation (1.1) converge monotonically to $x_{*}$ as $t \rightarrow \infty$. On the other hand, nonmonotone convergence and nonconvergent oscillation around $x_{*}$ arise in some rate control problems. Equation (1.1) neglects the feedback delays appearing naturally in the system.

The rate control model of Ranjan, La and Abed [26, 25] takes the feedback delays into account. As the rate $x(t)$ can be larger than the capacity of the server, the data arriving at the server may form a single waiting line (a queue) before processing. Let $y(t)$ denote the length of the queue at time $t$. Suppose that it is bounded from above by $q>0$, and the units of data reaching the queue with length $q$ are lost. Then, assuming that the transmission time from the user to the server is $r_{0} \geq 0$, it is natural that for the length $y(t)$ of the queue the differential equation

$$
\dot{y}(t)= \begin{cases}x\left(t-r_{0}\right)-c & \text { if } 0<y(t)<q, \\ {\left[x\left(t-r_{0}\right)-c\right]^{+}} & \text {if } y(t)=0 \\ -\left[x\left(t-r_{0}\right)-c\right]^{-} & \text {if } y(t)=q\end{cases}
$$

is satisfied. Here, equation (1.2) is required to hold almost everywhere, and $u^{+}=$ $\max \{u, 0\}, u^{-}=\max \{-u, 0\}$ denote the positive and negative parts of $u$, respectively.

Suppose that a unit of data, whose processing was completed and the user received an acknowledgement about it at time $t$, arrived at the queue $\tau(t)$ time earlier, i.e., at time $t-\tau(t)$, and found a queue with length $y(t-\tau(t))$. As the capacity of the server is $c$, the given unit of data spent waiting time $z(t)=(1 / c) y(t-\tau(t))$ in the queue before its processing started. Let $r_{1}$ denote the sum of the processing time and the transmission time from the server to the user. Then $\tau(t)=z(t)+r_{1}$, and this gives the algebraic equation

$$
z(t)=\frac{1}{c} y\left(t-z(t)-r_{1}\right)
$$

between $y$ and $z$. 
With the waiting time $z(t)$ and the transmission delays $r_{0}, r_{1}$, the user at time $t$ receives an acknowledgement from the server about the processing of that unit of data which was sent at time $t-r_{0}-z(t)-r_{1}$. The server determines a price for a unit rate when it arrives at the server, i.e., at time $t-z(t)-r_{1}$. When the processing of a unit ends, the server sends a signal to the user including the identification of the processed unit and the price information $p\left(x\left(t-z(t)-r_{1}\right)\right)$. Then the user is able to estimate the price for the rate of data sent at $t-r_{0}-z(t)-r_{1}$ as $x\left(t-r_{0}-z(t)-r_{1}\right) p\left(x\left(t-z(t)-r_{1}\right)\right)$. This led Ranjan, La and Abed [26, 25] to the rate control equation

$$
\dot{x}(t)=\kappa\left[x(t) U^{\prime}(x(t))-x\left(t-r_{0}-z(t)-r_{1}\right) p\left(x\left(t-z(t)-r_{1}\right)\right)\right]
$$

with a gain parameter $\kappa>0$. See figure 1 . For similar models we refer to $[1,3]$.

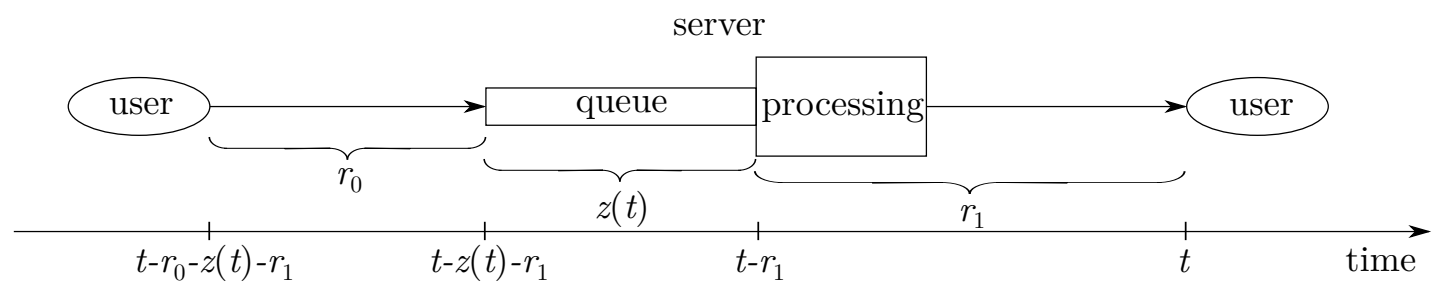

Figure 1: The process in time.

In this paper we consider rate control equations (like (1.4)) with delay, where the delay is determined by two auxiliary equations, by (1.3) and (1.2), or only by (1.2). The primary aim of this paper is to find a suitable framework to study the above types of rate control systems. We define a phase space where the corresponding initial value problem has a unique maximal solution. The solutions define a continuous semiflow, and the solution operators are Lipschitz continuous. We believe that this approach can be extended to handle a wide class of systems modeling networks with queueing delays. Observe that neither the classical results for equations with constant delay [7,9] nor the recently developed results for equations with state-dependent delay [10, 29] are applicable here.

The papers of Ranjan et al. [17, 15, 16, 23, 24, 25, 26] and [1, 3] consider similar systems, starting from discrete ones, through ordinary and delay differential equations with constant or state-dependent delays, to general network systems. They examine these models from the engineering point of view, comparing the different rate control schemes, like the TCP (transmission control protocol) and its modifications. In these papers, they prove local and global stability of equilibria, show bifurcations, and plot numerical solutions related to their results. However, as far as we know, they do not study the problem of existence, uniqueness, continuous dependence with a mathematical rigor.

The secondary aim is to apply the developed framework, and to show that the rate control defined by System (1.4), (1.2), (1.3) may lead to a slowly oscillating periodic rate around the optimal rate $x_{*}$, provided that the stationary solution $x=x_{*}, y=0, z=0$ is unstable and $r_{0}=0$. This answers affirmatively a conjecture of Ranjan and his coauthors $[24,22]$.

Before giving an overview on the main steps toward the results, some notation is introduced. $\mathbb{N}$ denotes the set of positive integers, $\mathbb{R}$ and $\mathbb{C}$ stand for the set of real and complex numbers, respectively. For $n \in \mathbb{N}, \mathbb{R}^{n}$ is the $n$-dimensional Euclidean space with norm $|\cdot|$. For a closed and bounded interval $I$ let $C_{I}$ denote the Banach space $C(I, \mathbb{R})$ 
equipped with the norm $\|\varphi\|_{I}=\max _{s \in I}|\varphi(s)|$. For Banach spaces $\mathcal{E}, \mathcal{F}$ with norms $\|\cdot\|_{\mathcal{E}}$, $\|\cdot\|_{\mathcal{F}}$, the norm on $\mathcal{E} \times \mathcal{F}$ is $\|(u, v)\|=\|u\|_{\mathcal{E}}+\|v\|_{\mathcal{F}}, u \in \mathcal{E}, v \in \mathcal{F}$.

Set $r=r_{0}+r_{1}+q / c>0$ as an upper bound for the delays. If $I \subseteq \mathbb{R}$ is an interval, $u: I \rightarrow \mathbb{R}$ is continuous, $r>0, t$ and $t-r$ are in $I$, then $u_{t} \in C_{[-r, 0]}$ is defined by $u_{t}(s)=u(t+s), s \in[-r, 0]$.

For a Lipschitz continuous $\varphi: I \rightarrow \mathbb{R}$, defined on the interval $I$, let

$$
\begin{gathered}
\operatorname{lip}(\varphi)=\sup _{s \in I, t \in I, s<t}\left|\frac{\varphi(t)-\varphi(s)}{t-s}\right| \in[0, \infty) \text { and } \\
\operatorname{slope}(\varphi)=\left\{\frac{\varphi(t)-\varphi(s)}{t-s}: s \in I, t \in I, s \neq t\right\} \subseteq \mathbb{R} .
\end{gathered}
$$

First we consider a slightly more general system than (1.4), (1.2), (1.3), that is, in the equation we allow more general dependence on the length of the queue than that of (1.4), (1.2), (1.3), and equation (1.3) may or may not hold. Consider the equation

$$
\dot{x}(t)=F\left(x_{t}, y_{t}\right)
$$

together with (1.2) in the phase space $X \times Y$ where $X, Y$ and $F$ are defined as follows. An upper bound $K>0$ for the absolute value of the right hand side of equation (1.5) comes from the nature of the problem. Then, by $x(t) \in[a, b]$ and the bound $K$, the subset

$$
X=\left\{\varphi \in C_{[-r, 0]} \mid \varphi([-r, 0]) \subseteq[a, b], \operatorname{lip}(\varphi) \leq K\right\}
$$

of $C_{[-r, 0]}$ will contain all possible segments $x_{t}$. Analogously, by $y(t) \in[0, q], x(t) \in[a, b]$ and equation (1.2), for the segments $y_{t}$, it is natural to introduce the subset

$$
Y=\left\{\psi \in C_{[-r, 0]} \mid \psi([-r, 0]) \subseteq[0, q], \operatorname{slope}(\psi) \subseteq[a-c, b-c]\right\}
$$

of $C_{[-r, 0]}$. On $X \subset C_{[-r, 0]}, Y \subset C_{[-r, 0]}, X \times Y \subset C_{[-r, 0]} \times C_{[-r, 0]}$ we use the induced subspace topologies and the corresponding norms. By the Arzelà-Ascoli theorem, $X, Y$ and $X \times Y$ are compact subsets of $C_{[-r, 0]}$ and $C_{[-r, 0]} \times C_{[-r, 0]}$, respectively. Assume that the map $F: X \times Y \rightarrow \mathbb{R}$ has the following properties:

(F1) there exists an $L>0$ such that, for all $\varphi^{1}, \varphi^{2} \in X, \psi^{1}, \psi^{2} \in Y$,

$$
\left|F\left(\varphi^{1}, \psi^{1}\right)-F\left(\varphi^{2}, \psi^{2}\right)\right| \leq L\left(\left\|\varphi^{1}-\varphi^{2}\right\|_{[-r, 0]}+\left\|\psi^{1}-\psi^{2}\right\|_{[-r, 0]}\right)
$$

(F2) $\max _{(\varphi, \psi) \in X \times Y}|F(\varphi, \psi)| \leq K$

(F3) there exists $r_{2} \in\left(0, r_{1}\right]$ such that $F\left(\varphi, \psi^{1}\right)=F\left(\varphi, \psi^{2}\right)$ provided $\varphi \in X, \psi^{1} \in Y$, $\psi^{2} \in Y$, and $\left.\psi^{1}\right|_{\left[-r,-r_{2}\right]}=\left.\psi^{2}\right|_{\left[-r,-r_{2}\right]} ;$

(F4) $F(\varphi, \psi)>0$ if $\varphi \in X, \psi \in Y, \varphi(0)=a$, and $F(\varphi, \psi)<0$ if $\varphi \in X, \psi \in Y$ and $\varphi(0)=b$.

A solution of System (1.5), (1.2) in the phase space $X \times Y$ on $[-r, \omega), \omega \leq \infty$, with initial condition $x_{0}=\varphi \in X, y_{0}=\psi \in Y$ is a pair of functions

$$
x=x^{\varphi, \psi}:[-r, \omega) \rightarrow \mathbb{R} \text { and } y=y^{\varphi, \psi}:[-r, \omega) \rightarrow \mathbb{R}
$$

such that 
(i) $x_{t} \in X$ for all $t \in[0, \omega), x_{0}=\varphi$;

(ii) $x$ is differentiable on $(0, \omega)$;

(iii) $y_{t} \in Y$ for all $t \in[0, \omega), y_{0}=\psi$;

(iv) equation (1.5) holds on $(0, \omega)$;

(v) equation (1.2) holds almost everywhere in $(0, \omega)$.

The solution $(x, y)=\left(x^{\varphi, \psi}, y^{\varphi, \psi}\right)$ on $[-r, \omega)$ is called maximal if any other solution $(\widehat{x}, \widehat{y})$ with $\widehat{x}_{0}=\varphi, \widehat{y}_{0}=\psi$ is a restriction of $(x, y)$.

In section 3 we show that, under hypotheses (F1)-(F4), for each $(\varphi, \psi) \in X \times Y$, system $(1.5),(1.2)$ has a unique maximal solution $\left(x^{\varphi, \psi}, y^{\varphi, \psi}\right):[-r, \infty) \rightarrow \mathbb{R}^{2}$. The solutions define the continuous semiflow

$$
\Phi:[0, \infty) \times X \times Y \ni(t, \varphi, \psi) \mapsto\left(x_{t}^{\varphi, \psi}, y_{t}^{\varphi, \psi}\right) \in X \times Y,
$$

and, for each $t \geq 0$, the solution operators $\Phi(t, \cdot, \cdot): X \times Y \rightarrow X \times Y$ are Lipschitz continuous (theorem 3.4). In order to sketch the main steps of the proof, let $(\varphi, \psi) \in X \times Y$ be given. As, by (F3), the value of $F(\varphi, \psi)$ does not depend on $\left.\psi\right|_{\left[-r_{2}, 0\right]}$, a standard contraction argument yields $T \in\left(0, r_{2}\right]$ and a unique $x:[-r, T] \rightarrow \mathbb{R}$ so that equation $(1.5)$ holds on $(0, T)$, for arbitrary extension of $y_{0}=\psi$ to $y:[-r, T] \rightarrow \mathbb{R}$. Next we redefine $y:[-r, T] \rightarrow \mathbb{R}$ on $(0, T]$ such that $y_{t} \in Y$ for all $t \in[0, T]$, and equation (1.2) holds almost everywhere on $[0, T]$ with $x:[-r, T] \rightarrow \mathbb{R}$ obtained in the first step. In order to appropriately redefine $y:[-r, T] \rightarrow \mathbb{R}$ on $[0, T]$, we extend the right hand side of (1.2) to an upper semicontinuous multivalued map, and apply a standard result from [6] for differential inclusions. These two steps combined give a unique solution $\left(x^{\varphi, \psi}, y^{\varphi, \psi}\right)$ on $[-r, T]$. By the method of steps the solution can be uniquely extended to a maximal solution on some $[-r, \omega)$. Global existence, i.e., $\omega=\infty$, follows from (F4).

In order to see that System (1.4), (1.2), (1.3) is a particular case of System (1.5), (1.2) introduce $Z=[0, q / c] \subset \mathbb{R}$ as a state space for the variable $z(t)$. A crucial fact is the existence of a unique Lipschitz continuous map (lemma 3.5) $\sigma: Y \rightarrow Z$, with Lipschitz constant $1 / a$, such that

$$
\sigma(\psi)=\frac{1}{c} \psi\left(-\sigma(\psi)-r_{1}\right) \quad(\psi \in Y)
$$

Then, for a solution $(x, y):[-r, \infty) \rightarrow \mathbb{R}^{2}$ of System (1.5), (1.2) in the phase space $X \times Y$, defining $z(t)=\sigma\left(y_{t}\right), t \geq 0$, equation (1.3) is always satisfied for all $t \geq 0$.

Define a map $G: X \times Z \rightarrow \mathbb{R}$ such that

(G1) there exists an $L_{G}>0$ such that, for all $\varphi^{1}, \varphi^{2} \in X, \zeta^{1}, \zeta^{2} \in Z$,

$$
\left|G\left(\varphi^{1}, \zeta^{1}\right)-G\left(\varphi^{2}, \zeta^{2}\right)\right| \leq L_{G}\left(\left\|\varphi^{1}-\varphi^{2}\right\|_{[-r, 0]}+\left|\zeta^{1}-\zeta^{2}\right|\right)
$$

(G2) $\max _{(\varphi, \zeta) \in X \times Z}|G(\varphi, \zeta)| \leq K$

(G3) $G(\varphi, \zeta)>0$ if $\varphi \in X, \zeta \in Z, \varphi(0)=a$, and $G(\varphi, \zeta)<0$ if $\varphi \in X, \zeta \in Z$ and $\varphi(0)=b$ 
hold. If we define $F$ by

$$
F: X \times Y \ni(\varphi, \psi) \mapsto G(\varphi, \sigma(\psi)) \in \mathbb{R},
$$

then it is straightforward to check that hypotheses (F1)-(F4) are satisfied provided $L_{G} \leq$ $L \min \{1, a\}$. In this case System (1.5), (1.2) is equivalent to the system composed of the equations

$$
\dot{x}(t)=G\left(x_{t}, z(t)\right),
$$

(1.2) and (1.3). Then, in the phase space $X \times Y$, for each $(\varphi, \psi) \in X \times Y$, System (1.6), $(1.2),(1.3)$ has the unique solution $x^{\varphi, \psi}[-r, \infty) \rightarrow \mathbb{R}, y^{\varphi, \psi}:[-r, \infty) \rightarrow \mathbb{R}, z^{\varphi, \psi}:[0, \infty) \rightarrow$ $\mathbb{R}$ where $\left(x^{\varphi, \psi}, y^{\varphi, \psi}\right)$ is the solution of System (1.5), (1.2) with the above choice of $F$, and $z^{\varphi, \psi}(t)=\sigma\left(y_{t}^{\varphi, \psi}\right), t \geq 0$.

Defining the map $G$ as

$$
G: X \times Z \ni(\varphi, \zeta) \mapsto \kappa\left[\varphi(0) U^{\prime}(\varphi(0))-\varphi\left(-\zeta-r_{0}-r_{1}\right) p\left(\varphi\left(-\zeta-r_{1}\right)\right)\right] \in \mathbb{R},
$$

System (1.4), (1.2), (1.3) will be a particular case of System (1.6), (1.2), (1.3), see section 5 .

In section 3 we show that System (1.6), (1.2), (1.3) can be studied not only in the phase space $X \times Y$, but also in $X \times Z$ with a different notion of solution. For given $(\varphi, \zeta) \in X \times Z$, the pair of functions $x:[-r, \infty) \rightarrow \mathbb{R}, z:[0, \infty) \rightarrow \mathbb{R}$ is called a solution of System (1.6), (1.2), (1.3) in the phase space $X \times Z$ if $x_{t} \in X$ and $z(t) \in Z$ for all $t \geq 0$, $x_{0}=\varphi, z(0)=\zeta, x$ is differentiable and equation (1.6) holds on $(0, \infty)$, moreover, there exists a function $y:[-r, \infty) \rightarrow \mathbb{R}$ with $y_{t} \in Y, z(t)=\sigma\left(y_{t}\right)$ for all $t \geq 0$, and equation (1.2) is satisfied almost everywhere on $\left[-\zeta-r_{1}, \infty\right)$.

The key technical result (see section 3) to show that System (1.6), (1.2), (1.3) is well posed in $X \times Z$ is that there is a unique Lipschitz continuous map $\gamma: X \times Z \rightarrow Y$ so that $\psi=\gamma(\varphi, \zeta)$ satisfies $\psi(s)=c \zeta$ for $s \in\left[-r,-\zeta-r_{1}\right]$, and equation (1.2) holds with $x(t)=\varphi(t), y(t)=\psi(t)$ a.e. in $\left[-\zeta-r_{1}, 0\right]$. In particular, $\zeta=(1 / c) \psi\left(-\zeta-r_{1}\right)$. This means that the past of the length of the queue (that is $\psi \in Y$ ) can be recovered from the past of the rate (that is $\varphi \in X$ ) and from the present waiting time (that is $\zeta \in Z$ ). The maps

$$
h: X \times Z \ni(\varphi, \zeta) \mapsto(\varphi, \gamma(\varphi, \zeta)) \in X \times Y, k: X \times Y \ni(\varphi, \psi) \mapsto(\varphi, \sigma(\psi)) \in X \times Z
$$

are Lipschitz continuous, $h$ is injective, and $k \circ h=\mathrm{id}_{X \times Z},\left.h \circ k\right|_{h(X \times Z)}=\mathrm{id}_{h(X \times Z)}$. Then (see theorem 3.10), for each $(\varphi, \zeta) \in X \times Z$, there exists a unique solution $x^{\varphi, \zeta}:[-r, \infty) \rightarrow$ $\mathbb{R}, z^{\varphi, \zeta}:[0, \infty) \rightarrow \mathbb{R}$ of System (1.6), (1.2), (1.3) in the phase space $X \times Z$ satisfying the initial condition $x_{0}^{\varphi, \zeta}=\varphi, z^{\varphi, \zeta}(0)=\zeta$. Moreover,

$$
\Psi:[0, \infty) \times X \times Z \ni(t, \varphi, \zeta) \mapsto\left(x_{t}^{\varphi, \zeta}, z^{\varphi, \zeta}(t)\right) \in X \times Z
$$

is a continuous semiflow on $X \times Z$, and $\Psi(t, \varphi, \zeta)=k(\Phi(t, h(\varphi, \zeta)))$ for all $t \geq 0$.

In the second part of the paper we study a particular system including the model of Ranjan et al. [26, 25], and show that the optimal equilibrium may be unstable, and in 
that case a slowly oscillatory periodic solution appears. Namely, we consider the system

$$
\begin{aligned}
& \dot{v}(t)=-f(v(t))-g(v(t-z(t)-1)) \\
& \dot{y}(t)= \begin{cases}v(t)-d & \text { if } 0<y(t)<q \\
{[v(t)-d]^{+}} & \text {if } y(t)=0 \\
-[v(t)-d]^{-} & \text {if } y(t)=q\end{cases} \\
& z(t)=\frac{1}{c} y(t-z(t)-1)
\end{aligned}
$$

with reals $c, q$ as before, and $A<0<d<B$, and nonlinearities $f, g$ in $C^{1}([A, B], \mathbb{R})$ satisfying $0 \leq f(\xi) / \xi \leq f_{1}, 0<g(\xi) / \xi \leq g_{1}$ for all $\xi \in[A, B] \backslash\{0\}$ for some $f_{1} \geq 0$, $g_{1}>0$.

Assume $r_{0}=0, r_{1}=1$ in equation (1.4). Condition $r_{0}=0$ guarantees a single delay in equation (1.4), $r_{1}=1$ can be achieved by rescaling the time. Then, under natural conditions on $U, p$ in equation (1.4), the rate control System (1.4), (1.2), (1.3) will be a particular case of System (1.8), (1.9), (1.10), see Sections 4-5.

Under suitable conditions on $f, g$, see section 4 , proposition 4.1 shows that System (1.8), (1.9), (1.10) is well posed in the phase space $\mathcal{X} \times Z$ where

$$
\mathcal{X}=\left\{\varphi \in C_{[-r, 0]} \mid \varphi([-r, 0]) \subseteq[A, B], \operatorname{lip}(\varphi) \leq K_{1}\right\},
$$

and $r=1+q / c, K_{0}=\left(f_{1}+g_{1}\right) \max \{-A, B\}, K_{1}=r K_{0}$.

A solution $(v, z)$ of System (1.8), (1.9), (1.10) is called slowly oscillatory if for any two zeros $t_{1}, t_{2}$ of $v$ with $t_{1}<t_{2}$ the inequality $z\left(t_{2}\right)+1<t_{2}-t_{1}$ holds. This means that the distance between consecutive zeros of $v$ is larger than the delay.

Inspired by [20] and [19], introduce

$$
W=\left\{(\varphi, \zeta) \in \mathcal{X} \times Z|\varphi|_{[-r,-\zeta-1]} \equiv 0, s \mapsto \varphi(s) e^{f_{1} s} \text { is nondecreasing, } \varphi(0)>0\right\}
$$

and $W_{0}=W \cup\{(0,0)\}$. Then, for each $(\varphi, \zeta) \in W$, the solution $v=v^{\varphi, \zeta}:[-r, \infty) \rightarrow \mathbb{R}$, $z=z^{\varphi, \zeta}:[0, \infty) \rightarrow \mathbb{R}$ is slowly oscillatory with an infinite number of zeros of $v$. The second zero $t_{2}$ of $v$ in $(0, \infty)$ determines $t_{2}^{*}>t_{2}$ so that $t_{2}=t_{2}^{*}-z\left(t_{2}^{*}\right)-1$, and a return map $P: W_{0} \rightarrow W_{0}$ can be defined by $P(0,0)=(0,0)$, and $P(\varphi, \zeta)=\Gamma\left(\Theta\left(t_{2}^{*}, \varphi, \zeta\right)\right)$ for $(\varphi, \zeta) \in W$ where $\Gamma: \mathcal{X} \times Z \rightarrow \mathcal{X} \times Z$ is given by $\Gamma(\varphi, \zeta)=(\widehat{\varphi}, \zeta)$, with $\widehat{\varphi}(s)=\varphi(s)$ for $s \in[-\zeta-1,0]$, and $\widehat{\varphi}(s)=\varphi(-\zeta-1)$ for $s \in[-r,-\zeta-1]$. A nontrivial fixed point of $P$ corresponds to a slowly oscillating periodic solution. A classical tool, that we apply here as well, is Browder's non-ejective fixed point theorem. A large part of section 4 is devoted to the construction of a suitable subset of $\mathcal{X} \times Z$ where Browder's theorem is applicable. We remark that, although the papers [20, 21, 2, 19, 31, 33, 30, 29] consider a similar approach to get slowly oscillating periodic solutions, none of them can be directly applied here, because of the particular definition of the state-dependent queueing delay. Some steps of the proof are analogous, and other parts require new ideas.

It is a crucial result that $P(\varphi, \zeta)$ cannot decay too fast: there are constants $\theta>0$, $\rho>0$ with $v^{\varphi, \zeta}\left(t_{2}^{*}\right) \geq \theta(\varphi(0))^{\rho}$ for all $(\varphi, \zeta) \in W$. This fact allows to construct a $C^{2}$ function $\alpha$ on $[0, q / c]$ such that $\alpha(0)=0, \alpha^{\prime}>0, \alpha^{\prime \prime}>0$ on $(0, q / c], \alpha(q / c)$ is small enough, and the delayed inequality

$$
\alpha\left(\xi-\frac{d}{c}\right) \geq \theta(\alpha(\xi))^{\rho} \quad\left(\xi \in\left[\frac{d}{c}, \frac{q}{c}\right]\right)
$$


holds, provided $d<q$. Defining the compact subsets

$$
\begin{aligned}
& W_{\alpha, K_{1}}=\left\{(\varphi, \zeta) \in W_{0} \mid \varphi(0) \geq \alpha(\zeta)\right\}, \\
& W_{\alpha, K_{0}}=\left\{(\varphi, \zeta) \in W_{\alpha, K_{1}} \mid \operatorname{lip}(\varphi) \leq K_{0}\right\}
\end{aligned}
$$

of $\mathcal{X} \times Z$, the inclusion $P\left(W_{\alpha, K_{1}}\right) \subseteq W_{\alpha, K_{0}}$ is satisfied. However, $W_{\alpha, K_{1}}$ and $W_{\alpha, K_{0}}$ are not convex. Following [19], the subset

$$
\begin{aligned}
& V_{\alpha, K_{1}}=\left\{(\psi, \zeta) \in C_{[-1,0]} \times Z \mid \psi([-1,0]) \subseteq[0, B], \operatorname{lip}(\psi) \leq K_{1},\right. \\
& \left.[-1,0] \ni s \mapsto \psi(s) e^{f_{1} r s} \in \mathbb{R} \text { is nondecreasing, } \psi(-1)=0, \psi(0) \geq \alpha(\zeta)\right\}
\end{aligned}
$$

of $C_{[-1,0]} \times \mathbb{R}$ is compact and convex. The set $V_{\alpha, K_{1}}$ can be mapped into $W_{\alpha, K_{1}}$ by the streching map $Q$ given by $Q(\psi, \zeta)=(\varphi, \zeta)$ with $\varphi(s)=\psi(s /(\zeta+1)), s \in[-\zeta-1,0]$, and $\left.\varphi\right|_{[-r,-\zeta-1]} \equiv 0$. The squeezing map $R$, defined by $R(\varphi, \zeta)=(\psi, \zeta)$ with $\psi(s)=\varphi((\zeta+1) s)$, $s \in[-1,0]$, maps $W_{\alpha, K_{0}}$ into $V_{\alpha, K_{1}}$. Browder's theorem can be applied for the map

$$
\Pi: V_{\alpha, K_{1}} \in(\psi, \zeta) \mapsto R \circ P \circ Q(\psi, \zeta) \in V_{\alpha, K_{1}}
$$

to find a non-ejective fixed point of $\Pi$ in $V_{\alpha, K_{1}}$. This yields a non-ejective fixed point of $P$ in $W_{\alpha, K_{1}}$ as well. The non-ejective fixed point is nontrivial provided $(0,0) \in W_{\alpha, K_{1}}$ is ejective. Ejectivity of $(0,0) \in W_{\alpha, K_{1}}$ follows in a standard way from that of the zero solution of the constant delay equation $\dot{v}(t)=-f(v(t))-g(v(t-1))$.

Finally, section 5 gives examples.

\section{Preliminary results}

In order to study the queue equation (1.2) we recall a basic result of [6] for differential inclusions.

Let $J=\left[t_{0}, t_{1}\right] \subset \mathbb{R}$ for some fixed $t_{0}, t_{1} \in \mathbb{R}, t_{0}<t_{1}$, and let $D \subseteq \mathbb{R}^{j}$ be closed. The multivalued map

$$
F: J \times D \rightarrow 2^{\mathbb{R}^{j}} \backslash\{\emptyset\}=\left\{A \subseteq \mathbb{R}^{j}, A \neq \emptyset\right\}
$$

is called upper semicontinuous if $F^{-1}(A)$ is closed in $J \times D$ whenever $A \subseteq \mathbb{R}^{j}$ is closed. Note that the definition of the inverse image

$$
F^{-1}(A)=\{(t, y) \in J \times D \mid F(t, y) \cap A \neq \emptyset\}
$$

is different from that of a single valued map.

Let $\rho(y, D)=\inf _{z \in D}|y-z|$ for $y \in \mathbb{R}^{j}$. For $y \in D$ define

$$
T_{D}(y)=\left\{z \in \mathbb{R}^{j}: \liminf _{\lambda \rightarrow 0^{+}} \frac{1}{\lambda} \rho(y+\lambda z, D)=0\right\} .
$$

The following existence result is Theorem 5.1 in [6]:

Theorem A. Suppose that the multivalued map $F: J \times D \rightarrow 2^{\mathbb{R}^{j}} \backslash\{\emptyset\}$ is upper semicontinuous, for all $(t, y) \in J \times D$ the set $F(t, y)$ is closed and convex in $\mathbb{R}^{j}$,

$$
F(t, y) \cap T_{D}(y) \neq \emptyset \quad \text { for all }(t, y) \in J \times D,
$$


moreover, there is a Lebesgue integrable $c: J \rightarrow[0, \infty)$ such that, for all $(t, y) \in J \times D$,

$$
\sup \{|z|: z \in F(t, y)\} \leq c(t)(1+|y|)
$$

holds. Then, for each $y_{0} \in D$, there exists an absolutely continuous $y: J \rightarrow D$ such that $y\left(t_{0}\right)=y_{0}$ and the inclusion $\dot{y}(t) \in F(t, y(t))$ holds a.e. on $\left[t_{0}, t_{1}\right]$.

Assume that $\mathcal{E}$ is a Banach space, $\mathcal{C} \subset \mathcal{E}$ is compact and convex in $\mathcal{E}$, the map $\mathcal{F}: \mathcal{C} \rightarrow \mathcal{C}$ is continuous. A fixed point $x_{0} \in \mathcal{C}$ of $\mathcal{F}$ is said to be ejective if there exists an open neighborhood $\mathcal{U}$ of $x_{0}$ in $\mathcal{C}$ such that for each $x \in \mathcal{U} \backslash\left\{x_{0}\right\}$ there exists a positive integer $k(x)$ such that for the iterate $\mathcal{F}^{k(x)}(x) \in \mathcal{C} \backslash \mathcal{U}$ holds. In section 4 we will apply the following result of Browder [4] on the existence of a non-ejective fixed point.

Theorem B. Assume that $\mathcal{E}$ is a Banach space, $\mathcal{C} \subset \mathcal{E}$ is an infinite dimensional compact and convex subset of $\mathcal{E}$, the map $\mathcal{F}: \mathcal{C} \rightarrow \mathcal{C}$ is continuous. Then $\mathcal{F}$ has a non-ejective fixed point.

For the application of the above result, we have to guarantee the ejectivity of the trivial fixed point of a return map. The proof of the ejectivity uses properties of the linear autonomous equation with constant delay

$$
\dot{w}(t)=-\mu w(t)-\nu w(t-1)
$$

where $\mu \geq 0$ and $\nu>0$. We recall some basic results from [7, 9, 32]. It is well known that every $\varphi \in C_{[-1,0]}$ uniquely determines a solution $w^{\varphi}:[-1, \infty) \rightarrow \mathbb{R}$ of equation (2.2) with $\left.w^{\varphi}\right|_{[-1,0]}=\varphi$, and the solutions define the strongly continuous semigroup $(T(t))_{t \geq 0}$ on $[0, \infty) \times C_{[-1,0]}$. The spectrum of the generator consists of the solutions $\lambda \in \mathbb{C}$ of the characteristic equation $\lambda+\mu+\nu e^{-\lambda}=0$. Assume $\nu>e^{-\mu-1}$. Then all points in the spectrum form a sequence of complex conjugate pairs $\left(\lambda_{j}, \overline{\lambda_{j}}\right)_{j=1}^{\infty}$ with $\operatorname{Re} \lambda_{j}>\operatorname{Re} \lambda_{j+1}, \operatorname{Im} \lambda_{j} \in((2 j-2) \pi,(2 j-1) \pi)$ for all $j \in \mathbb{N}$, and $\operatorname{Re} \lambda_{j} \rightarrow-\infty$ as $j \rightarrow \infty$. An explicit criterion for $\operatorname{Re} \lambda_{1}>0$ is

$$
\nu>\frac{\vartheta}{\sin \vartheta} \text { where } \vartheta \in(0, \pi) \text { is the unique solution of } \mu=-\vartheta \cot \vartheta .
$$

Let $\mathcal{L}$ and $\mathcal{Q}$ denote the realified generalized eigenspaces of the generator associated with the spectral sets $\left\{\lambda_{1}, \overline{\lambda_{1}}\right\}$ and $\left\{\lambda_{k}, \overline{\lambda_{k}}: k \geq 2\right\}$, respectively. Then $C_{[-1,0]}=\mathcal{L} \oplus \mathcal{Q}$. A basis of $\mathcal{L}$ is given by the restrictions of the functions

$$
t \mapsto e^{\operatorname{Re} \lambda_{1} t} \sin \left(\operatorname{Im} \lambda_{1} t\right), \quad t \mapsto e^{\operatorname{Re} \lambda_{1} t} \cos \left(\operatorname{Im} \lambda_{1} t\right)
$$

to the interval $[-1,0]$.

Let $S \subset C_{[-1,0]} \backslash\{0\}$ be the set of functions with at most one sign change in $[-1,0]$. The set $S$ is invariant, i.e., $T(t) S \subseteq S$ for all $t \geq 0$. Moreover, $S \cap \mathcal{Q}=\emptyset$.

Proposition 2.1. If $\mu \geq 0$ and $\nu>0$ are given such that $\operatorname{Re} \lambda_{1}>0$, i.e., inequality (2.3) holds, and $\varphi \in S$, then the solution $w^{\varphi}$ of equation $(2.2)$ is unbounded on $[-1, \infty)$.

Proof. Let $\varphi \in S$ and $w=w^{\varphi}$. From $C_{[-1,0]}=\mathcal{L} \oplus \mathcal{Q}, S \cap \mathcal{Q}=\emptyset$ and $\varphi \neq 0$ it follows that $\varphi=\varphi^{\mathcal{L}}+\varphi^{\mathcal{Q}}$ with $\varphi^{\mathcal{L}} \in \mathcal{L} \backslash\{0\}, \varphi^{\mathcal{Q}} \in \mathcal{Q}$. Then $w=w_{\mathcal{L}}+w_{\mathcal{Q}}$ where $w_{\mathcal{L}}=w^{\varphi^{\mathcal{L}}}$ and $w_{\mathcal{Q}}=w^{\varphi^{\mathcal{Q}}}$. As $\varphi^{\mathcal{L}} \in \mathcal{L} \backslash\{0\}$, there exist $k_{1}, k_{2} \in \mathbb{R}$ with $k_{1}^{2}+k_{2}^{2} \neq 0$ so that, for all $t \geq-1$

$$
w_{\mathcal{L}}(t)=e^{\operatorname{Re} \lambda_{1} t}\left[k_{1} \sin \left(\operatorname{Im} \lambda_{1} t\right)+k_{2} \cos \left(\operatorname{Im} \lambda_{1} t\right)\right] .
$$


The estimate on the complementary space $\mathcal{Q}$ (see, e.g., [7] or [9]) implies that there are $\delta>0$ and $M>0$ such that, for all $t \geq-1$,

$$
\left|w_{\mathcal{Q}}(t)\right| \leq M e^{\left(\operatorname{Re} \lambda_{1}-\delta\right) t} .
$$

Then, by $\operatorname{Re} \lambda_{1}>0$, it easly follows that $w$ is unbounded.

\section{The solution semiflow}

Assume that $r_{0}, r_{1}, r_{2}, q, a, b, c, K, L$ are given constants as in section 1 , and Hypotheses (F1) $-(\mathrm{F} 4)$ hold. First we consider System (1.5), (1.2). Condition (F3) means that $F(\varphi, \psi)$ does not depend on $\left.\psi\right|_{\left[-r_{2}, 0\right]}$. Consequently, for given $\varphi \in X$ and $\psi \in Y$, we can find $x:[-r, T] \rightarrow \mathbb{R}$ with $x_{0}=\varphi$ satisfying equation (1.5) on an interval $[0, T]$ for some $T \in\left(0, r_{2}\right)$, no matter how $\left.y\right|_{[-r, 0]}=\psi$ is extended to $[-r, T]$. This is done in the next proposition by using a standard fixed point technique. After that $x:[-r, T] \rightarrow \mathbb{R}$ is obtained, we will be able to determine $y:[-r, T] \rightarrow \mathbb{R}$ satisfying equation $(1.2)$ on $[0, T]$. These two results together give a solution of System (1.5), (1.2) on $[-r, T]$. Repeating this procedure by time- $T$ steps a global solution will be obtained.

Proposition 3.1. Let $T \in\left(0, r_{2}\right]$ be fixed such that $T L<1$. For every $(\varphi, \psi) \in X \times Y$ there exists a unique function $x=x(\varphi, \psi):[-r, T] \rightarrow \mathbb{R}$ such that $x_{0}=\varphi, x_{t} \in X$ for all $t \in[0, T], x$ is differentiable on $(0, T]$, and, for each $y:[-r, T] \rightarrow \mathbb{R}$ with $y_{0}=\psi$ and $y_{t} \in Y$ for all $t \in[0, T], x$ satisfies equation (1.5) on $(0, T]$. Moreover, the Lipschitz continuity property

$$
\left\|x\left(\varphi^{1}, \psi^{1}\right)-x\left(\varphi^{2}, \psi^{2}\right)\right\|_{[-r, T]} \leq \frac{\left\|\varphi^{1}-\varphi^{2}\right\|_{[-r, 0]}+T L\left\|\psi^{1}-\psi^{2}\right\|_{[-r, 0]}}{1-T L}
$$

holds for all $\left(\varphi^{1}, \psi^{1}\right),\left(\varphi^{2}, \psi^{2}\right)$ in $X \times Y$.

Proof. Let $(\varphi, \psi) \in X \times Y$ be given. Define $\widehat{\varphi}, \widehat{\psi} \in C_{[-r, T]}$ by $\widehat{\varphi}(t)=\varphi(t), \widehat{\psi}(t)=\psi(t)$ for $t \in[-r, 0]$, and $\widehat{\varphi}(t)=\varphi(0), \widehat{\psi}(t)=\psi(0)$ for $t \in[0, T]$.

The set

$$
M=\left\{u \in C_{[0, T]}: u(0)=0, \operatorname{lip}(u) \leq K\right\},
$$

is a complete metric space with distance $d(u, v)=\|u-v\|_{[0, T]}$. Introduce the map $m: M \times[a, b] \rightarrow C_{[-r, T]}$ by

$$
m(u, \xi)(t)= \begin{cases}0 & \text { if } t \in[-r, 0], \\ \min \{\max \{u(t), a-\xi\}, b-\xi\} & \text { if } t \in[0, T] .\end{cases}
$$

The function $m(u, \xi)$ is a trivial extension of $u$ to $[-r, 0]$, and it cuts the values of $u$ on $[0, T]$ so that $m(u, \xi)(t) \in[a-\xi, b-\xi]$ is satisfied. Then it is clear that

$$
\widehat{\varphi}_{t}+m_{t}(u, \varphi(0)) \in X, \quad \widehat{\psi}_{t} \in Y \text { for all } t \in[0, T],
$$

and $[0, T] \ni t \mapsto \widehat{\varphi}_{t}+m_{t}(u, \varphi(0)) \in X,[0, T] \ni t \mapsto \widehat{\psi}_{t} \in Y$ are continuous maps. It is easy to see that

$$
\left\|m\left(u^{1}, \xi\right)-m\left(u^{2}, \xi\right)\right\|_{[-r, T]} \leq\left\|u^{1}-u^{2}\right\|_{[0, T]} \quad\left(u^{1} \in M, u^{2} \in M, \xi \in[a, b]\right) .
$$


Define the map $\mathcal{N}: X \times Y \times M \rightarrow M$ as follows:

$$
\mathcal{N}(\varphi, \psi, u)(t)=\int_{0}^{t} F\left(\widehat{\varphi}_{s}+m_{s}(u, \varphi(0)), \widehat{\psi}_{s}\right) d s, \quad t \in[0, T]
$$

By (F1) and (F2), $F$ is continuous and $|F| \leq K$. Therefore, it is obvious that $\mathcal{N}(\varphi, \psi, u) \in$ $M$.

Now, fix $(\varphi, \psi) \in X \times Y$. For functions $u^{1}, u^{2} \in M$, by the definition of $\mathcal{N}, m$, and by (F1) and the Lipschitz property of $m$, we have

$$
\begin{aligned}
& \left\|\mathcal{N}\left(\varphi, \psi, u^{1}\right)-\mathcal{N}\left(\varphi, \psi, u^{2}\right)\right\|_{[0, T]} \\
& =\max _{t \in[0, T]}\left|\int_{0}^{t}\left[F\left(\widehat{\varphi}_{s}+m_{s}\left(u^{1}, \varphi(0)\right), \widehat{\psi}_{s}\right)-F\left(\widehat{\varphi}_{s}+m_{s}\left(u^{2}, \varphi(0)\right), \widehat{\psi}_{s}\right)\right] d s\right| \\
& \leq \int_{0}^{T} L\left\|m\left(u^{1}, \varphi(0)\right)-m\left(u^{2}, \varphi(0)\right)\right\|_{[-r, T]} d s \leq T L\left\|u^{1}-u^{2}\right\|_{[0, T]} .
\end{aligned}
$$

Since $T L<1$, for all $(\varphi, \psi) \in X \times Y$, the map $M \ni u \mapsto \mathcal{N}(\varphi, \psi, u) \in M$ is a contraction. Therefore, as $M$ is a complete metric space, there is a unique fixed point $u^{*}(\varphi, \psi) \in M$.

Let $\left(\varphi^{i}, \psi^{i}\right) \in X \times Y$ and $u_{i}^{*}=u^{*}\left(\varphi^{i}, \psi^{i}\right), i=1,2$. From the obvious inequality

$$
\left\|\widehat{\varphi}^{1}+m\left(u, \varphi^{1}(0)\right)-\widehat{\varphi}^{2}-m\left(u, \varphi^{2}(0)\right)\right\|_{[-r, T]} \leq\left\|\varphi^{1}-\varphi^{2}\right\|_{[-r, 0]},
$$

it follows that

$$
\begin{aligned}
& \left\|u_{1}^{*}-u_{2}^{*}\right\|_{[0, T]}=\left\|\mathcal{N}\left(\varphi^{1}, \psi^{1}, u_{1}^{*}\right)-\mathcal{N}\left(\varphi^{2}, \psi^{2}, u_{2}^{*}\right)\right\|_{[0, T]} \\
& \leq \max _{t \in[0, T]}\left|\int_{0}^{t}\left[F\left(\widehat{\varphi}_{s}^{1}+m_{s}\left(u_{1}^{*}, \varphi^{1}(0)\right), \widehat{\psi}_{s}^{1}\right)-F\left(\widehat{\varphi}_{s}^{2}+m_{s}\left(u_{2}^{*}, \varphi^{2}(0)\right), \widehat{\psi}_{s}^{2}\right)\right] d s\right| \\
& \leq \int_{0}^{T} L\left(\left\|\widehat{\varphi}^{1}+m\left(u_{1}^{*}, \varphi^{1}(0)\right)-\widehat{\varphi}^{2}-m\left(u_{1}^{*}, \varphi^{2}(0)\right)\right\|_{[-r, T]}\right. \\
& \left.+\left\|m\left(u_{1}^{*}, \varphi^{2}(0)\right)-m\left(u_{2}^{*}, \varphi^{2}(0)\right)\right\|_{[-r, T]}+\left\|\widehat{\psi}^{1}-\widehat{\psi}^{2}\right\|_{[-r, T]}\right) d s \\
& \leq T L\left(\left\|\varphi^{1}-\varphi^{2}\right\|_{[-r, 0]}+\left\|u_{1}^{*}-u_{2}^{*}\right\|_{[0, T]}+\left\|\psi^{1}-\psi^{2}\right\|_{[-r, 0]}\right) .
\end{aligned}
$$

Consequently,

$$
\left\|u^{*}\left(\varphi^{1}, \psi^{1}\right)-u^{*}\left(\varphi^{2}, \psi^{2}\right)\right\|_{[0, T]} \leq \frac{T L}{1-T L}\left(\left\|\varphi^{1}-\varphi^{2}\right\|_{[-r, 0]}+\left\|\psi^{1}-\psi^{2}\right\|_{[-r, 0]}\right) .
$$

We claim that $\varphi(0)+u^{*}(\varphi, \psi)(t) \in(a, b)$ for all $(\varphi, \psi) \in X \times Y, t \in(0, T]$.

If $t_{0} \in[0, T]$ and $\varphi(0)+u^{*}(\varphi, \psi)\left(t_{0}\right)=a$, we have $\widehat{\varphi}\left(t_{0}\right)+m\left(u^{*}(\varphi, \psi), \varphi(0)\right)\left(t_{0}\right)=a$. Then by $(\mathrm{F} 4), F\left(\widehat{\varphi}_{t_{0}}+m_{t_{0}}\left(u^{*}(\varphi, \psi), \varphi(0)\right), \widehat{\psi}_{t_{0}}\right)>0$. By continuity, it follows that there is a $\delta>0$ so that $F\left(\widehat{\varphi}_{t}+m_{t}\left(u^{*}(\varphi, \psi), \varphi(0)\right), \widehat{\psi}_{t}\right)>0$ for all $t \in\left(t_{0}-\delta, t_{0}+\delta\right) \cap[0, T]$. The fixed point equation for $u^{*}(\varphi, \psi)$ implies that $t \mapsto u^{*}(\varphi, \psi)(t)$ strictly increases in $\left(t_{0}-\delta, t_{0}+\delta\right) \cap[0, T]$. Hence it is easy to see that

$$
\varphi(0)+u^{*}(\varphi, \psi)(t)>a \text { for all } t \in(0, T] .
$$

Analogously, $\varphi(0)+u^{*}(\varphi, \psi)(t)<b$ holds for all $t \in(0, T]$. So the claim is true. 
A consequence of the claim is that

$$
m\left(u^{*}(\varphi, \psi), \varphi(0)\right)(t)=u^{*}(\varphi, \psi)(t) \text { for all } t \in[0, T]
$$

and the function

$$
x(t)=x(\varphi, \psi)(t)= \begin{cases}\varphi(t) & \text { if } t \in[-r, 0], \\ \varphi(0)+u^{*}(\varphi, \psi)(t) & \text { if } t \in[0, T]\end{cases}
$$

satisfies $x_{0}=\varphi, x_{t} \in X$ for $t \in[0, T], x$ is differentiable on $(0, T]$, and equation (1.5) holds on $(0, T]$ with the particular choice $y=\widehat{\psi}$. Observe that, by hypothesis (F3), the above construction gives the same $x(\varphi, \psi)$ for any $y:[-r, T] \rightarrow \mathbb{R}$ so that $y_{0}=\psi$ and $y_{t} \in Y$ for $t \in[0, T]$.

Finally, it is straightforward to get the estimate

$$
\begin{aligned}
\left\|x\left(\varphi^{1}, \psi^{1}\right)-x\left(\varphi^{2}, \psi^{2}\right)\right\|_{[-r, T]} & \leq\left\|\varphi^{1}-\varphi^{2}\right\|_{[-r, 0]}+\left\|u^{*}\left(\varphi^{1}, \psi^{1}\right)-u^{*}\left(\varphi^{2}, \psi^{2}\right)\right\|_{[0, T]} \\
& \leq \frac{1}{1-T L}\left\|\varphi^{1}-\varphi^{2}\right\|_{[-r, 0]}+\frac{T L}{1-T L}\left\|\psi^{1}-\psi^{2}\right\|_{[-r, 0]} .
\end{aligned}
$$

This completes the proof.

In the next step we study equation (1.2). Since we need the same type of result in another situation as well, a slightly more general version is considered.

Let $t_{0}, t_{1} \in \mathbb{R}$ with $t_{0}<t_{1}$. Assume that a function $\xi \in C\left(\left[t_{0}, t_{1}\right],[a, b]\right)$ is given. Let $y^{0} \in[0, q]$ be fixed. We consider the equation

$$
\dot{y}(t)= \begin{cases}\xi(t)-c & \text { if } 0<y(t)<q, \\ {[\xi(t)-c]^{+}} & \text {if } y(t)=0 \\ -[\xi(t)-c]^{-} & \text {if } y(t)=q\end{cases}
$$

on the interval $\left[t_{0}, t_{1}\right]$ with initial condition $y\left(t_{0}\right)=y^{0}$.

Proposition 3.2. For each $\xi \in C\left(\left[t_{0}, t_{1}\right],[a, b]\right)$ and each $y^{0} \in[0, q]$ there exists a unique Lipschitz continuous function $y=y\left(\xi, y^{0}\right):\left[t_{0}, t_{1}\right] \rightarrow[0, q]$ such that $y\left(t_{0}\right)=y^{0}, \operatorname{slope}(y) \subseteq$ $[a-c, b-c]$, and equation (3.1) holds almost everywhere in $\left[t_{0}, t_{1}\right]$. In addition, $y\left(\xi, y^{0}\right)$ is Lipschitz continuous in $\xi, y^{0}$, namely, for all $\xi^{1}, \xi^{2} \in C\left(\left[t_{0}, t_{1}\right],[a, b]\right)$ and $y^{0,1}, y^{0,2} \in[0, q]$,

$$
\left\|y\left(\xi^{1}, y^{0,1}\right)-y\left(\xi^{2}, y^{0,2}\right)\right\|_{\left[t_{0}, t_{1}\right]} \leq\left|y^{0,1}-y^{0,2}\right|+\left(t_{1}-t_{0}\right)\left\|\xi^{1}-\xi^{2}\right\|_{\left[t_{0}, t_{1}\right]} .
$$

Proof. Let $\xi \in C\left(\left[t_{0}, t_{1}\right],[a, b]\right)$ and $y^{0} \in[0, q]$ be fixed. Define the map $h:\left[t_{0}, t_{1}\right] \times[0, q] \rightarrow$ $\mathbb{R}$ by

$$
h(t, y)= \begin{cases}\xi(t)-c & \text { if } 0<y<q \\ {[\xi(t)-c]^{+}} & \text {if } y=0 \\ -[\xi(t)-c]^{-} & \text {if } y=q .\end{cases}
$$

Then equation (3.1) with $y\left(t_{0}\right)=y^{0}$ on $\left[t_{0}, t_{1}\right]$ can be written as an initial value problem

$$
\left\{\begin{array}{l}
\dot{y}(t)=h(t, y(t)) \quad \text { a.e. for } t \in\left[t_{0}, t_{1}\right] \\
y\left(t_{0}\right)=y^{0}
\end{array}\right.
$$


The remaining part of the proof is divided into three steps. In Steps 1-2 we show existence, in Step 3 uniqueness and the Lipschitz property are obtained.

Step 1 . We extend $h$ to a multivalued function $\widetilde{h}:\left[t_{0}, t_{1}\right] \times[0, q] \rightarrow 2^{\mathbb{R}} \backslash\{\emptyset\}$ as follows:

$$
\widetilde{h}(t, y)= \begin{cases}\{\xi(t)-c\} & \text { if } y \in(0, q) \\ & \text { or } y=0 \text { and } \xi(t) \geq c \\ & \text { or } y=q \text { and } \xi(t) \leq c \\ {[\xi(t)-c, 0]} & \text { if } y=0 \text { and } \xi(t)<c \\ {[0, \xi(t)-c]} & \text { if } y=q \text { and } \xi(t)>c\end{cases}
$$

We claim that $\widetilde{h}$ is an upper semicontinuous function. To check it, let $A \subseteq \mathbb{R}$ be closed, nonempty, and consider its inverse image $\widetilde{h}^{-1}(A)$ defined by $(2.1)$ with $F=\widetilde{h}$. Let $\left(s_{n}, y_{n}\right)_{n=0}^{\infty}$ be a sequence in $\widetilde{h}^{-1}(A)$ converging to $\left(s_{*}, y_{*}\right) \in\left[t_{0}, t_{1}\right] \times[0, q]$. We have to show that $\left(s_{*}, y_{*}\right) \in \widetilde{h}^{-1}(A)$, i.e., $\widetilde{h}\left(s_{*}, y_{*}\right) \cap A$ is nonempty. By the definition of $\widetilde{h}$, $\xi(t)-c \in \widetilde{h}(t, y)$. Thus $\xi\left(s_{*}\right)-c \in \widetilde{h}\left(s_{*}, y_{*}\right)$, and, since $\xi$ is continuous and $A$ is closed, we have

$$
\widetilde{h}\left(s_{n}, y_{n}\right) \ni \xi\left(s_{n}\right)-c \rightarrow \xi\left(s_{*}\right)-c \in A .
$$

Therefore $\widetilde{h}^{-1}(A)$ is closed.

We apply theorem A by choosing $j=1, D=[0, q], J=\left[t_{0}, t_{1}\right], F=\widetilde{h}$. Clearly, $T_{D}(y)=\mathbb{R}$ for $y \in(0, q), T_{D}(0)=[0, \infty)$ and $T_{D}(q)=(-\infty, 0]$. It is obvious that the conditions of theorem $\mathrm{A}$ are satisfied with $c(t)=\max \{c-a, b-c\}$. Therefore, there is an absolutely continuous $y=y\left(\xi, y^{0}\right):\left[t_{0}, t_{1}\right] \rightarrow[0, q]$ such that

$$
\dot{y}(t) \in \widetilde{h}(t, y(t)) \quad \text { a.e. for } t \in\left[t_{0}, t_{1}\right]
$$

and $y\left(t_{0}\right)=y^{0}$.

Step 2. We show that for the function $y=y\left(\xi, y^{0}\right)$, obtained in Step 1, equation (3.1) holds almost everywhere, and $y\left(t_{0}\right)=y^{0}$.

Assume that $t \in\left(t_{0}, t_{1}\right)$ is given such that $\dot{y}(t)$ exists and $\dot{y}(t) \in \widetilde{h}(t, y(t))$.

If $y(t) \in(0, q)$ then $\widetilde{h}(t, y(t))=\{\xi(t)-c\}$, and consequently $\dot{y}(t)=h(t, y(t))$. If $y(t)=0$ then necessarily $\dot{y}(t)=0$. From $\dot{y}(t)=0 \in \widetilde{h}(t, 0)$ it follows that $\xi(t) \leq c$, and thus $0=\dot{y}(t)=[\xi(t)-c]^{+}=h(t, y(t))$. The case $y(t)=q$ is analogous.

Therefore, $y=y\left(\xi, y^{0}\right)$ satisfies equation (3.2). Then, by the definition of $h(t, y)$ and $\xi\left(\left[t_{0}, t_{1}\right]\right) \subseteq[a, b]$, it is clear that $(3.1)$ holds almost everywhere for $y, y\left(t_{0}\right)=y^{0}$, and slope $(y) \subseteq[a-c, b-c]$.

Step 3. Let $\xi^{1}, \xi^{2} \in C\left(\left[t_{0}, t_{1}\right],[a, b]\right), y^{0,1}, y^{0,2} \in[0, q], y^{1}=y\left(\xi^{1}, y^{0,1}\right), y^{2}=y\left(\xi^{2}, y^{0,2}\right)$. Then the map $\left[t_{0}, t_{1}\right] \ni t \mapsto\left|y^{1}(t)-y^{2}(t)\right| \in \mathbb{R}$ is absolutely continuous.

Claim. For $\xi^{1}, \xi^{2} \in C\left(\left[t_{0}, t_{1}\right],[a, b]\right), y^{0,1}, y^{0,2} \in[0, q], y^{1}=y\left(\xi^{1}, y^{0,1}\right), y^{2}=y\left(\xi^{2}, y^{0,2}\right)$,

$$
\frac{d}{d s}\left|y^{1}(s)-y^{2}(s)\right| \leq\left|\xi^{1}(s)-\xi^{2}(s)\right| \quad \text { holds a.e. in }\left[t_{0}, t_{1}\right] \text {. }
$$

Observe that, for almost all $s \in\left(t_{0}, t_{1}\right)$, the derivative $\dot{y}^{i}(s)$ exists with $\dot{y}^{i}(s)=h^{i}\left(s, y^{i}(s)\right)$, where $h^{i}$ is the map constructed as $h$ above with $\xi$ replaced by $\xi^{i}, i=1,2$, moreover, $\left(t_{0}, t_{1}\right) \ni s \mapsto\left|y^{1}(s)-y^{2}(s)\right| \in \mathbb{R}$ is differentiable almost everywhere. Fix such an $s \in\left(t_{0}, t_{1}\right)$. Remark that if a real function $\alpha$ and its absolute value $|\alpha|$ are differentiable at $s$, then

$$
\frac{d}{d s}|\alpha(s)|=\lim _{\delta \rightarrow 0} \frac{|\alpha(s+\delta)|-|\alpha(s)|}{\delta} \leq\left|\lim _{\delta \rightarrow 0} \frac{\alpha(s+\delta)-\alpha(s)}{\delta}\right|=|\dot{\alpha}(s)| .
$$


We distinguish 4 cases.

Case 1. $y^{i}(s) \in(0, q), i \in\{1,2\}$. Then, by (3.4) and the definition of $h^{1}, h^{2}$,

$$
\frac{d}{d s}\left|y^{1}(s)-y^{2}(s)\right| \leq\left|\dot{y}^{1}(s)-\dot{y}^{2}(s)\right|=\left|\xi^{1}(s)-\xi^{2}(s)\right| .
$$

Case 2. $y^{i}(s) \in\{0, q\}, i \in\{1,2\}$. In this case $\dot{y}^{1}(s)=\dot{y}^{2}(s)=0$, and hence

$$
\frac{d}{d s}\left|y^{1}(s)-y^{2}(s)\right| \leq\left|\dot{y}^{1}(s)-\dot{y}^{2}(s)\right|=0 \leq\left|\xi^{1}(s)-\xi^{2}(s)\right|
$$

Case 3. $y^{1}(s)=0, y^{2}(s) \in(0, q)$. Then $\dot{y}^{1}(s)=0$ and consequently $\xi^{1}(s) \leq c$. In addition,

$$
\begin{aligned}
\frac{d}{d s}\left|y^{1}(s)-y^{2}(s)\right| & =\frac{d}{d s}\left(y^{2}(s)-y^{1}(s)\right)=\dot{y}^{2}(s)-\dot{y}^{1}(s) \\
& =\xi^{2}(s)-c \leq \xi^{2}(s)-\xi^{1}(s) \leq\left|\xi^{1}(s)-\xi^{2}(s)\right| .
\end{aligned}
$$

Case 4. $y^{1}(s) \in(0, q), y^{2}(s)=q$. Then $\dot{y}^{2}(s)=0$ and $\xi^{2}(s) \geq c$ follow. Hence

$$
\begin{aligned}
\frac{d}{d s}\left|y^{1}(s)-y^{2}(s)\right| & =\frac{d}{d s}\left(y^{2}(s)-y^{1}(s)\right)=\dot{y}^{2}(s)-\dot{y}^{1}(s) \\
& =-\left(\xi^{1}(s)-c\right)=c-\xi^{1}(s) \leq \xi^{2}(s)-\xi^{1}(s) \leq\left|\xi^{1}(s)-\xi^{2}(s)\right| .
\end{aligned}
$$

The remaining cases can be obtained by changing the indices. This completes the proof of the claim.

By the definition of the norm, the absolute continuity of $t \mapsto\left|y^{1}(t)-y^{2}(t)\right|$, and by the above claim, we have

$$
\begin{aligned}
\left\|y^{1}-y^{2}\right\|_{\left[t_{0}, t_{1}\right]} & =\max _{t \in\left[t_{0}, t_{1}\right]}\left|y^{1}(t)-y^{2}(t)\right| \\
& =\max _{t \in\left[t_{0}, t_{1}\right]}\left(\left|y^{0,1}-y^{0,2}\right|+\int_{t_{0}}^{t} \frac{d}{d s}\left|y^{1}(s)-y^{2}(s)\right| d s\right) \\
& \leq\left|y^{0,1}-y^{0,2}\right|+\max _{t \in\left[t_{0}, t_{1}\right]} \int_{t_{0}}^{t}\left|\xi^{1}(s)-\xi^{2}(s)\right| d s \\
& \leq\left|y^{0,1}-y^{0,2}\right|+\left(t_{1}-t_{0}\right)\left\|\xi^{1}-\xi^{2}\right\|_{\left[t_{0}, t_{1}\right]} .
\end{aligned}
$$

This implies the uniqueness of $y\left(\xi, y^{0}\right)$, and the Lipschitz continuity of $y\left(\xi, y^{0}\right)$ with respect to $\xi$ and $y^{0}$. The proof is complete.

The following corollary is immediate from lemma 3.2.

Corollary 3.3. Let $T>0$. For all $\widetilde{\xi} \in C([-r, T],[a, b])$ and $\psi \in Y$ there exists a unique Lipschitz continuous function $y=y(\widetilde{\xi}, \psi):[-r, T] \rightarrow[0, q]$ such that $y_{0}=\psi$, slope $(y) \subseteq$ $[a-c, b-c]$, and equation (3.1) holds with $\xi(t)=\widetilde{\xi}\left(t-r_{0}\right)$ almost everywhere in $[0, T]$. In addition, $y(\widetilde{\xi}, \psi)$ is Lipschitz continuous in $\widetilde{\xi}, \psi$, namely, for all $\widetilde{\xi}, \widetilde{\xi} \widetilde{\xi}^{2} \in C([-r, T],[a, b])$ and $\psi^{1}, \psi^{2} \in Y$,

$$
\left\|y\left(\widetilde{\xi}^{1}, \psi^{1}\right)-y\left(\widetilde{\xi^{2}}, \psi^{2}\right)\right\|_{[-r, T]} \leq\left\|\psi^{1}-\psi^{2}\right\|_{[-r, 0]}+T\left\|\widetilde{\xi}^{1}-\widetilde{\xi}^{2}\right\|_{[-r, T]} .
$$

Now we are in a position to prove existence, uniqueness, and continuous dependence of the solutions of System (1.5), (1.2). 
Theorem 3.4. For each $(\varphi, \psi) \in X \times Y$ there exists a unique solution

$$
x^{\varphi, \psi}:[-r, \infty) \rightarrow \mathbb{R}, \quad y^{\varphi, \psi}:[0, \infty) \rightarrow \mathbb{R}
$$

of System (1.5), (1.2) on $[-r, \infty)$ satisfying the initial condition $x_{0}^{\varphi, \psi}=\varphi, y_{0}^{\varphi, \psi}=\psi$. The mapping

$$
\Phi:[0, \infty) \times X \times Y \ni(t, \varphi, \psi) \mapsto\left(x_{t}^{\varphi, \psi}, y_{t}^{\varphi, \psi}\right) \in X \times Y
$$

defines a continuous semiflow on $X \times Y$. In addition, for all $\left(\varphi^{j}, \psi^{j}\right) \in X \times Y, j=1,2$, $t \geq 0, \Phi$ has the Lipschitz continuity property

$$
\left\|\Phi\left(t, \varphi^{1}, \psi^{1}\right)-\Phi\left(t, \varphi^{2}, \psi^{2}\right)\right\|_{X \times Y} \leq\left\|\left(\varphi^{1}, \psi^{1}\right)-\left(\varphi^{2}, \psi^{2}\right)\right\|_{X \times Y} e^{t(1+L)} .
$$

Proof. Let $T \in\left(0, r_{2}\right], T L<1$ and $(\varphi, \psi) \in X \times Y$. By proposition 3.1 there exists a unique function $x=x(\varphi, \psi):[-r, T] \rightarrow \mathbb{R}$ such that $x_{0}=\varphi, x_{t} \in X$ for all $t \in[0, T], x$ is differentiable on $(0, T], x$ satisfies equation $(1.5)$ on $(0, T]$, and the function $y:[-r, T] \rightarrow \mathbb{R}$ in (1.5) is arbitrary with $y_{0}=\psi$ and $y_{t} \in Y$ for all $t \in[0, T]$. By corollary 3.3, with $\widetilde{\xi}=x(\varphi, \psi)$, we can choose a unique $y=y(x(\varphi, \psi), \psi):[-r, T] \rightarrow \mathbb{R}$ such that $y_{0}=\psi$, $y_{t} \in Y$ for all $t \in[0, T]$, and equation (1.2) holds almost everywhere in $[0, T]$.

The functions $x^{\varphi, \psi}:[-r, \infty) \rightarrow \mathbb{R}$ and $y^{\varphi, \psi}:[0, \infty) \rightarrow \mathbb{R}$ are defined as follows. Set $x^{\varphi, \psi}(t)=x(\varphi, \psi)(t), y^{\varphi, \psi}(t)=y(x(\varphi, \psi), \psi)(t)$ for $t \in[-r, T]$. Hence we can define $\widetilde{\varphi}=x_{T}^{\varphi, \psi} \in X$ and $\widetilde{\psi}=y_{T}^{\varphi, \psi} \in Y$. For $(\widetilde{\varphi}, \widetilde{\psi}) \in X \times Y$, the functions $x(\widetilde{\varphi}, \widetilde{\psi})$ and $y(\widetilde{\varphi}, \widetilde{\psi})$ can be constructed as above. Set $x^{\varphi, \psi}(t)=x(\widetilde{\varphi}, \widetilde{\psi})(t-T), y^{\varphi, \psi}(t)=y(x(\widetilde{\varphi}, \widetilde{\psi}), \widetilde{\psi})(t-T)$ for $t \in[T, 2 T]$. This procedure can be repeated to define $x^{\varphi, \psi}$ and $y^{\varphi, \psi}$ on the interval $[-r, \infty)$. The differentiability of $x^{\varphi, \psi}$ on $(0, \infty)$ follows from the continuity of the map $[0, \infty) \ni t \mapsto F\left(x_{t}^{\varphi, \psi}, y_{t}^{\varphi, \psi}\right) \in \mathbb{R}$. Therefore the pair $x^{\varphi, \psi}, y^{\varphi, \psi}$ is a solution. In order to show uniqueness, let $\widehat{x}, \widehat{y}:[-r, \omega) \rightarrow \mathbb{R}$ be another solution with $\widehat{x}_{0}=\varphi, \widehat{y}_{0}=\psi$, and $0<\omega \leq \infty$. Pick a maximal $t_{0} \in[0, \omega)$ such that $x(t)=\widehat{x}(t), y(t)=\widehat{y}(t)$ for all $t \in\left[-r, t_{0}\right]$. Choosing $\left(x_{t_{0}}, y_{t_{0}}\right) \in X \times Y$ as initial pair of functions, proposition 3.1 and corollary 3.3 give that $x$ and $y$ are unique on the interval $\left[-r, t_{0}+\delta\right)$ for some $\delta>0$, respectively, a contradiction. So, the uniqueness holds. Define

$$
\Phi(t, \varphi, \psi)=\left(x_{t}^{\varphi, \psi}, y_{t}^{\varphi, \psi}\right) \quad(t \geq 0, \varphi \in X, \psi \in Y) .
$$

The semigroup property of $\Phi$ follows from the existence, uniqueness and from the fact that System (1.5), (1.2) is autonomous.

Now we prove that $\Phi$ is Lipschitz continuous in $\varphi, \psi$.

Let $\left(\varphi^{i}, \psi^{i}\right) \in X \times Y, x^{i}=x^{\varphi^{i}, \psi^{i}}, y^{i}=y^{\varphi^{i}, \psi^{i}}, i=1,2$. For each $T \geq 0$ with $T \in\left(0, r_{2}\right]$ and $T L<1$, by using proposition 3.1 and corollary 3.3, we have the estimate

$$
\begin{aligned}
& \left\|x^{1}-x^{2}\right\|_{[t-r, t+T]}+\left\|y^{1}-y^{2}\right\|_{[t-r, t+T]} \\
& \leq\left\|x^{1}-x^{2}\right\|_{[t-r, t+T]}+\left\|y^{1}-y^{2}\right\|_{[t-r, t]}+T\left\|x^{1}-x^{2}\right\|_{[t-r, t+T]} \\
& =(1+T)\left\|x^{1}-x^{2}\right\|_{[t-r, t+T]}+\left\|y^{1}-y^{2}\right\|_{[t-r, t]} \\
& \leq \frac{1+T}{1-T L}\left(\left\|x^{1}-x^{2}\right\|_{[t-r, t]}+T L\left\|y^{1}-y^{2}\right\|_{[t-r, t]}\right)+\left\|y^{1}-y^{2}\right\|_{[t-r, t]} \\
& =\frac{1+T}{1-T L}\left\|x^{1}-x^{2}\right\|_{[t-r, t]}+\frac{1+T^{2} L}{1-T L}\left\|y^{1}-y^{2}\right\|_{[t-r, t]} \\
& \leq \frac{1+T}{1-T L}\left(\left\|x^{1}-x^{2}\right\|_{[t-r, t]}+\left\|y^{1}-y^{2}\right\|_{[t-r, t]}\right) .
\end{aligned}
$$


Hence, for the right-hand upper Dini derivative we find

$$
\begin{aligned}
& D^{+}\left(\left\|x_{t}^{1}-x_{t}^{2}\right\|_{[-r, 0]}+\left\|y_{t}^{1}-y_{t}^{2}\right\|_{[-r, 0]}\right) \\
& =\limsup _{T \rightarrow 0+} \frac{1}{T}\left(\left\|x_{t+T}^{1}-x_{t+T}^{2}\right\|_{[-r, 0]}+\left\|y_{t+T}^{1}-y_{t+T}^{2}\right\|_{[-r, 0]}\right. \\
& \left.-\left\|x_{t}^{1}-x_{t}^{2}\right\|_{[-r, 0]}-\left\|y_{t}^{1}-y_{t}^{2}\right\|_{[-r, 0]}\right) \\
& \leq \limsup _{T \rightarrow 0+} \frac{1}{T}\left(\frac{1+T}{1-T L}\left(\left\|x_{t}^{1}-x_{t}^{2}\right\|_{[-r, 0]}+\left\|y_{t}^{1}-y_{t}^{2}\right\|_{[-r, 0]}\right)\right. \\
& \left.-\left\|x_{t}^{1}-x_{t}^{2}\right\|_{[-r, 0]}-\left\|y_{t}^{1}-y_{t}^{2}\right\|_{[-r, 0]}\right) \\
& \leq \limsup _{T \rightarrow 0+} \frac{1+L}{1-T L}\left(\left\|x_{t}^{1}-x_{t}^{2}\right\|_{[-r, 0]}+\left\|y_{t}^{1}-y_{t}^{2}\right\|_{[-r, 0]}\right) \\
& =(1+L)\left(\left\|x_{t}^{1}-x_{t}^{2}\right\|_{[-r, 0]}+\left\|y_{t}^{1}-y_{t}^{2}\right\|_{[-r, 0]}\right) .
\end{aligned}
$$

Then the inequality

$$
\begin{aligned}
& D^{+}\left[e^{-(L+1) t}\left(\left\|x_{t}^{1}-x_{t}^{2}\right\|_{[-r, 0]}+\left\|y_{t}^{1}-y_{t}^{2}\right\|_{[-r, 0]}\right)\right] \\
& =-(L+1) e^{-(L+1) t}\left(\left\|x_{t}^{1}-x_{t}^{2}\right\|_{[-r, 0]}+\left\|y_{t}^{1}-y_{t}^{2}\right\|_{[-r, 0]}\right) \\
& +e^{-(L+1) t} D^{+}\left(\left\|x_{t}^{1}-x_{t}^{2}\right\|_{[-r, 0]}+\left\|y_{t}^{1}-y_{t}^{2}\right\|_{[-r, 0]}\right) \leq 0
\end{aligned}
$$

easily follows for all $t \geq 0$. By Zygmund's inequality (see e.g., [28, p. 10] or [18, p. 9]) the function

$$
[0, \infty) \ni t \mapsto e^{-(L+1) t}\left(\left\|x_{t}^{1}-x_{t}^{2}\right\|_{[-r, 0]}+\left\|y_{t}^{1}-y_{t}^{2}\right\|_{[-r, 0]}\right) \in \mathbb{R}
$$

is monotone nonincreasing. Consequently,

$$
\left\|x_{t}^{1}-x_{t}^{2}\right\|_{[-r, 0]}+\left\|y_{t}^{1}-y_{t}^{2}\right\|_{[-r, 0]} \leq e^{(L+1) t}\left(\left\|x_{0}^{1}-x_{0}^{2}\right\|_{[-r, 0]}+\left\|y_{0}^{1}-y_{0}^{2}\right\|_{[-r, 0]}\right)
$$

for all $t \geq 0$. This shows the stated Lipschitz property of $\Phi$.

The continuity of $x^{\varphi, \psi}, y^{\varphi, \psi}:[-r, \infty) \rightarrow \mathbb{R}$ implies the continuity of the maps $[0, \infty) \ni$ $t \mapsto x_{t}^{\varphi, \psi} \in X,[0, \infty) \ni t \mapsto y_{t}^{\varphi, \psi} \in Y$, since the topology of $C_{[-r, 0]}$ is used on $X, Y$. Thus, for each $(\varphi, \psi) \in X \times Y$, the map $[0, \infty) \ni t \mapsto \Phi(t, \varphi, \psi) \in X \times Y$ is continuous.

Finally, by the inequality

$$
\begin{aligned}
& \left\|\Phi\left(t_{1}, \varphi^{1}, \psi^{1}\right)-\Phi\left(t_{2}, \varphi^{2}, \psi^{2}\right)\right\|_{X \times Y} \\
& \leq\left\|\Phi\left(t_{1}, \varphi^{1}, \psi^{1}\right)-\Phi\left(t_{1}, \varphi^{2}, \psi^{2}\right)\right\|_{X \times Y}+\left\|\Phi\left(t_{1}, \varphi^{2}, \psi^{2}\right)-\Phi\left(t_{2}, \varphi^{2}, \psi^{2}\right)\right\|_{X \times Y},
\end{aligned}
$$

the continuity of $\Phi$ follows from the above statements.

Now, we turn to the study of System (1.6), (1.2), (1.3), and prove that it can be considered not only in the phase space $X \times Y$ but also in $X \times Z$, see the definition of solutions in $X \times Z$.

First we show that equation (1.3) can be solved uniquely provided $y_{t} \in Y$. 
Proposition 3.5. There is a unique map $\sigma: Y \rightarrow Z$ satisfying

$$
\sigma(\psi)=\frac{1}{c} \psi\left(-\sigma(\psi)-r_{1}\right)
$$

The map $\sigma: Y \rightarrow Z$ is Lipschitz continuous, namely, for all $\psi^{1}, \psi^{2} \in Y$, we have

$$
\begin{aligned}
\left|\sigma\left(\psi^{1}\right)-\sigma\left(\psi^{2}\right)\right| & \leq \frac{1}{a} \max _{s \in\left[-\max \left\{\sigma\left(\psi^{1}\right), \sigma\left(\psi^{2}\right)\right\}-r_{1},-r_{1}\right]}\left|\psi^{1}(s)-\psi^{2}(s)\right| \\
& \leq \frac{1}{a}\left\|\psi^{1}-\psi^{2}\right\|_{[-r, 0]} .
\end{aligned}
$$

Proof. Let $\psi \in Y$ be given. Define $\varrho:[0, q / c] \ni s \mapsto s-\psi\left(-s-r_{1}\right) / c$. For $0 \leq s_{1}<s_{2} \leq$ $q / c$, by using slope $(\psi) \subseteq[a-c, b-c]$ and $0<a<c$, it follows that

$$
\begin{aligned}
\frac{\varrho\left(s_{1}\right)-\varrho\left(s_{2}\right)}{s_{1}-s_{2}} & =\frac{s_{1}-\psi\left(-s_{1}-r_{1}\right) / c-s_{2}+\psi\left(-s_{2}-r_{1}\right) / c}{s_{1}-s_{2}} \\
& =1-\frac{1}{c} \frac{\psi\left(-s_{1}-r_{1}\right)-\psi\left(-s_{2}-r_{1}\right)}{s_{1}-s_{2}} \\
& =1+\frac{1}{c} \frac{\psi\left(-s_{1}-r_{1}\right)-\psi\left(-s_{2}-r_{1}\right)}{\left(-s_{1}-r_{1}\right)-\left(-s_{2}-r_{1}\right)} \geq 1+\frac{a-c}{c}=\frac{a}{c}>0 .
\end{aligned}
$$

Hence, function $\varrho$ is strictly increasing in $[0, q / c]$. Observe that

$$
\varrho(0)=-\frac{\psi\left(-r_{1}\right)}{c} \leq 0 \quad \text { and } \quad \varrho\left(\frac{q}{c}\right)=\frac{q}{c}-\frac{\psi\left(q / c-r_{1}\right)}{c} \geq \frac{q}{c}-\frac{q}{c}=0 .
$$

So, $\varrho$ has a unique zero, denoted by $\sigma(\psi)$, in $[0, q / c]$. Clearly, $\sigma(\psi)$ is unique with $(3.5)$.

In order to prove the Lipschitz continuity of $\sigma$, let $\psi^{1}, \psi^{2} \in Y$ be given. If $\sigma\left(\psi^{1}\right)=$ $\sigma\left(\psi^{2}\right)$ then inequality (3.6) trivially holds. Without loss of generality assume $\sigma\left(\psi^{1}\right)>$ $\sigma\left(\psi^{2}\right)$. By slope $\left(\psi^{2}\right) \subseteq[a-c, b-c]$ we obtain

$$
\begin{aligned}
& \left|\sigma\left(\psi^{1}\right)-\sigma\left(\psi^{2}\right)\right|=\sigma\left(\psi^{1}\right)-\sigma\left(\psi^{2}\right)=\frac{\psi^{1}\left(-\sigma\left(\psi^{1}\right)-r_{1}\right)-\psi^{2}\left(-\sigma\left(\psi^{2}\right)-r_{1}\right)}{c} \\
& =\frac{\psi^{1}\left(-\sigma\left(\psi^{1}\right)-r_{1}\right)-\psi^{2}\left(-\sigma\left(\psi^{1}\right)-r_{1}\right)}{c}+\frac{\psi^{2}\left(-\sigma\left(\psi^{1}\right)-r_{1}\right)-\psi^{2}\left(-\sigma\left(\psi^{2}\right)-r_{1}\right)}{c} \\
& \leq \frac{1}{c} \max _{s \in\left[-\sigma\left(\psi^{1}\right)-r_{1},-r_{1}\right]}\left|\psi^{1}(s)-\psi^{2}(s)\right|+\frac{c-a}{c}\left|\sigma\left(\psi^{1}\right)-\sigma\left(\psi^{2}\right)\right| .
\end{aligned}
$$

Hence

$$
\left(1-\frac{c-a}{c}\right)\left|\sigma\left(\psi^{1}\right)-\sigma\left(\psi^{2}\right)\right| \leq \frac{1}{c} \max _{s \in\left[-\max \left\{\sigma\left(\psi^{1}\right), \sigma\left(\psi^{2}\right)\right\}-r_{1},-r_{1}\right]}\left|\psi^{1}(s)-\psi^{2}(s)\right|,
$$

from which inequality (3.6) easily holds.

The next proposition is a key technical result. It shows that, for given $\varphi \in X$ and $\zeta \in Z$, we can find uniquely an element $\psi \in Y$ such that $\psi$ satisfies equation (1.2), with $x=\varphi$ and $y=\psi$ a.e. in $\left[-\zeta-r_{1}, 0\right]$, and $\zeta=\sigma(\psi)$ holds as well. In order to guarantee the uniqueness of $\psi$ we choose it to have the constant value $c \zeta$ on $\left[-r,-\zeta-r_{1}\right]$. 
Proposition 3.6. There is a unique map

$$
\gamma: X \times Z \rightarrow Y
$$

so that $\psi=\gamma(\varphi, \zeta)$ satisfies

$$
\left\{\begin{array}{l}
\psi(s)=c \zeta \quad \text { for } s \in\left[-r,-\zeta-r_{1}\right] \\
\dot{\psi}(s)= \begin{cases}\varphi\left(s-r_{0}\right)-c & \text { if } 0<\psi(s)<q, \\
{\left[\varphi\left(s-r_{0}\right)-c\right]^{+}} & \text {if } \psi(s)=0, \\
-\left[\varphi\left(s-r_{0}\right)-c\right]^{-} & \text {if } \psi(s)=q\end{cases}
\end{array}\right.
$$

In addition, $\zeta=\sigma(\gamma(\varphi, \zeta))$ for all $(\varphi, \zeta) \in X \times Z$, and

$$
\left\|\gamma\left(\varphi^{1}, \zeta^{1}\right)-\gamma\left(\varphi^{2}, \zeta^{2}\right)\right\|_{[-r, 0]} \leq r\left\|\varphi^{1}-\varphi^{2}\right\|_{[-r, 0]}+\max \{2 c-a, b\}\left|\zeta^{1}-\zeta^{2}\right|
$$

for all $\varphi^{1}, \varphi^{2} \in X$ and $\zeta^{1}, \zeta^{2} \in Z$.

Proof. Let $(\varphi, \zeta) \in X \times Z$ be given. Define a function $\psi:[-r, 0] \rightarrow \mathbb{R}$ as follows. Let $\psi(s)=c \zeta$ for $s \in\left[-r, \zeta-r_{1}\right]$. Applying lemma 3.2 with $\left[t_{0}, t_{1}\right]=\left[-\zeta-r_{1}, 0\right]$, $\xi(s)=\varphi\left(s-r_{0}\right)$ for $s \in\left[-\zeta-r_{1}, 0\right], y^{0}=c \zeta$, we can uniquely define $\psi(s)=y\left(\xi, y^{0}\right)(s)$ for $s \in\left[-\zeta-r_{1}, 0\right]$. It is clear that $\gamma(\varphi, \zeta)=\psi$ is the unique element of $Y$ satisfying equation (3.7).

By the definition of $\gamma(\varphi, \zeta)$, we have $\zeta=(1 / c) \gamma(\varphi, \zeta)\left(-\zeta-r_{1}\right)$, that is $\zeta=\sigma(\gamma(\varphi, \zeta))$.

In order to show the Lipschitz continuity of $\gamma$, let $\left(\varphi^{i}, \zeta^{i}\right) \in X \times Z$ and $\psi^{i}=\gamma\left(\varphi^{i}, \zeta^{i}\right)$, $i=1,2$. Without loss of generality, assume that $\zeta^{1} \geq \zeta^{2}$. If $-r \leq s \leq-\zeta^{1}-r_{1}$ then

$$
\left|\psi^{1}(s)-\psi^{2}(s)\right|=\left|c \zeta^{1}-c \zeta^{2}\right|=c\left|\zeta^{1}-\zeta^{2}\right|
$$

If $-\zeta^{1}-r_{1} \leq s \leq-\zeta^{2}-r_{1}$ then, by using that $\psi^{1}$ is absolutely continuous (because it is Lipschitz continuous) and thus $\psi^{1}(s)-c \zeta^{1}=\int_{-\zeta^{1}-r_{1}}^{s} \dot{\psi}^{1}(u) d u$,

$$
\begin{aligned}
& \left|\psi^{1}(s)-\psi^{2}(s)\right|=\left|c \zeta^{1}+\int_{-\zeta^{1}-r_{1}}^{s} \dot{\psi}^{1}(u) d u-c \zeta^{2}\right| \\
& \leq\left|c \zeta^{1}-c \zeta^{2}\right|+\int_{-\zeta^{1}-r_{1}}^{-\zeta^{2}-r_{1}}\left|\varphi^{1}\left(u-r_{0}\right)-c\right| d u \\
& \leq c\left|\zeta^{1}-\zeta^{2}\right|+\max \{c-a, b-c\}\left|\zeta^{1}-\zeta^{2}\right|=\max \{2 c-a, b\}\left|\zeta^{1}-\zeta^{2}\right| .
\end{aligned}
$$

For $s \in\left[\zeta^{2}-r_{1}, 0\right]$ apply lemma 3.2 with $\left[t_{0}, t_{1}\right]=\left[-\zeta_{2}-r_{1}, 0\right], y(t)=\psi^{j}(t), \xi(t)=$ $\varphi^{j}\left(t-r_{0}\right), y^{0, j}=\psi^{j}\left(-\zeta^{2}-r_{1}\right), j=1,2$ to obtain

$$
\begin{aligned}
\left\|\psi^{1}-\psi^{2}\right\|_{\left[-\zeta^{2}-r_{1}, q\right]} & \leq\left|\psi^{1}\left(-\zeta^{2}-r_{1}\right)-\psi^{2}\left(-\zeta^{2}-r_{1}\right)\right| \\
& +\left(\zeta^{2}+r_{1}\right)\left\|\varphi^{1}-\varphi^{2}\right\|_{\left[-\zeta^{2}-r_{1}-r_{0},-r_{0}\right]}
\end{aligned}
$$

Combining (3.8), (3.9), (3.10), and using $c<2 c-a, \zeta^{2}+r_{1}+r_{0} \leq r$, we get the stated Lipschitz continuity.

Proposition 3.7. Let $y \in C([-r, \infty),[0, q])$ be a Lipschitz continuous function with $\operatorname{slope}(y) \subseteq[a-c, b-c]$. Then the function $z:[0, \infty) \ni t \mapsto \sigma\left(y_{t}\right) \in \mathbb{R}$ satisfies $\operatorname{slope}(z) \subseteq[1-c / a, 1-c / b]$. 
Proof. Clearly, $y_{t} \in Y$ and $z(t)=\sigma\left(y_{t}\right)=(1 / c) y\left(t-\sigma\left(y_{t}\right)-r_{1}\right)=(1 / c) y\left(t-z(t)-r_{1}\right)$, $t \geq 0$. Choose $t_{1} \geq 0, t_{2} \geq 0$ with $t_{1} \neq t_{2}$. Then $t_{1}-z\left(t_{1}\right)=t_{2}-z\left(t_{2}\right)$ implies $z\left(t_{1}\right)=(1 / c) y\left(t_{1}-z\left(t_{1}\right)\right)=(1 / c) y\left(t_{2}-z\left(t_{2}\right)\right)=z\left(t_{2}\right)$, and $t_{1}=t_{2}$, a contradiction. So, $t_{1}-z\left(t_{1}\right) \neq t_{2}-z\left(t_{2}\right)$, and

$$
\begin{aligned}
& \frac{z\left(t_{1}\right)-z\left(t_{2}\right)}{t_{1}-t_{2}}=\frac{1}{c} \frac{y\left(t_{1}-z\left(t_{1}\right)-r_{1}\right)-y\left(t_{2}-z\left(t_{2}\right)-r_{1}\right)}{t_{1}-t_{2}} \\
& =\frac{1}{c} \frac{y\left(t_{1}-z\left(t_{1}\right)-r_{1}\right)-y\left(t_{2}-z\left(t_{2}\right)-r_{1}\right)}{\left(t_{1}-z\left(t_{1}\right)-r_{1}\right)-\left(t_{2}-z\left(t_{2}\right)-r_{1}\right)} \frac{\left.\left(t_{1}\right)-r_{1}\right)-\left(t_{2}-z\left(t_{2}\right)-r_{1}\right)}{t_{1}-t_{2}} \\
& =\frac{1}{c} \frac{y\left(s_{1}\right)-y\left(s_{2}\right)}{s_{1}-s_{2}}\left(1-\frac{z\left(t_{1}\right)-z\left(t_{2}\right)}{t_{1}-t_{2}}\right)
\end{aligned}
$$

with $s_{j}=t_{j}-z\left(t_{j}\right)-r_{1}, j=1,2$. Rearranging terms and multiplying by $c$ we obtain

$$
\left(c+\frac{y\left(s_{1}\right)-y\left(s_{2}\right)}{s_{1}-s_{2}}\right) \frac{z\left(t_{1}\right)-z\left(t_{2}\right)}{t_{1}-t_{2}}=\frac{y\left(s_{1}\right)-y\left(s_{2}\right)}{s_{1}-s_{2}} .
$$

Using slope $(y) \subseteq[a-c, b-c]$, and $\xi /(c+\xi) \in[(a-c) / a,(b-c) / b]$ for $\xi \in[a-c, b-c]$, it follows that

$$
\frac{z\left(t_{1}\right)-z\left(t_{2}\right)}{t_{1}-t_{2}}=\frac{\frac{y\left(s_{1}\right)-y\left(s_{2}\right)}{s_{1}-s_{2}}}{c+\frac{y\left(s_{1}\right)-y\left(s_{2}\right)}{s_{1}-s_{2}}} \in\left[\frac{a-c}{a}, \frac{b-c}{b}\right]=\left[1-\frac{c}{a}, 1-\frac{c}{b}\right],
$$

and the proof is complete.

Proposition 3.8. Let $y \in C([-r, \infty),[0, q])$ be a Lipschitz continuous function with $\operatorname{slope}(y) \subseteq[a-c, b-c]$, and define $z:[0, \infty) \ni t \mapsto \sigma\left(y_{t}\right) \in \mathbb{R}$. Then the map

$$
\eta:[0, \infty) \ni t \mapsto t-z(t)-r_{1} \in \mathbb{R}
$$

is Lipschitz continuous with $\operatorname{slope}(\eta) \subseteq[c / b, c / a]$. In particular, $\eta$ is a strictly increasing function, and, for its inverse $\eta^{-1}$, slope $\left(\eta^{-1}\right) \subseteq[a / c, b / c]$ holds.

Proof. From lemma 3.7, with $t_{1} \geq 0, t_{2} \geq 0$ and $t_{1} \neq t_{2}$ we have

$$
\frac{\eta\left(t_{1}\right)-\eta\left(t_{2}\right)}{t_{1}-t_{2}}=\frac{\left(t_{1}-z\left(t_{1}\right)-r_{1}\right)-\left(t_{2}-z\left(t_{2}\right)-r_{1}\right)}{t_{1}-t_{2}}=1-\frac{z\left(t_{1}\right)-z\left(t_{2}\right)}{t_{1}-t_{2}} \in\left[\frac{c}{b}, \frac{c}{a}\right] .
$$

Let $t_{j}=\eta\left(s_{j}\right), j=1,2$, with $t_{1} \neq t_{2}$. Then, for the inverse

$$
\frac{\eta^{-1}\left(t_{1}\right)-\eta^{-1}\left(t_{2}\right)}{t_{1}-t_{2}}=\frac{\eta^{-1}\left(\eta\left(s_{1}\right)\right)-\eta^{-1}\left(\eta\left(s_{1}\right)\right)}{\eta\left(s_{1}\right)-\eta\left(s_{1}\right)}=\frac{s_{1}-s_{2}}{\eta\left(s_{1}\right)-\eta\left(s_{1}\right)} \in\left[\frac{a}{c}, \frac{b}{c}\right],
$$

completing the proof.

In the remaining part of this section we consider a map $G: X \times Z \rightarrow \mathbb{R}$ satisfying (G1)-(G3). Recall that, for $F: X \times Y \rightarrow \mathbb{R}$ given by

$$
F(\varphi, \psi)=G(\varphi, \sigma(\psi))
$$

Hypotheses (F1)-(F4) hold provided $L_{G} \geq L \min \{1, a\}$. We consider the system composed of equations

$$
\dot{x}(t)=G\left(x_{t}, \sigma\left(y_{t}\right)\right)
$$


and (1.2) in the phase space $X \times Y$ as it is a particular case of System (1.5), (1.2).

Define the mappings

$$
\begin{aligned}
& h: X \times Z \ni(\varphi, \zeta) \mapsto(\varphi, \gamma(\varphi, \zeta)) \in X \times Y, \\
& k: X \times Y \ni(\varphi, \psi) \mapsto(\varphi, \sigma(\psi)) \in X \times Z .
\end{aligned}
$$

Note that both of them are Lipschitz continuous, $h$ is injective, but $k$ is not. For their compositions, we have

$$
k \circ h=\operatorname{id}_{X \times Z} \quad \text { and }\left.\quad h \circ k\right|_{h(X \times Z)}=\operatorname{id}_{h(X \times Z)} .
$$

Proposition 3.9. If $\varphi \in X, \psi^{1} \in Y, \psi^{2} \in Y, \zeta \in Z$ with $\zeta=\sigma\left(\psi^{1}\right)=\sigma\left(\psi^{2}\right)$ and

$$
\psi^{1}(s)=\psi^{2}(s) \quad \text { for all } s \in\left[-\zeta-r_{1}, 0\right],
$$

then, for the semiflow $\Phi$ generated by System (3.11), (1.2), we have

$$
k\left(\Phi\left(t, \varphi, \psi^{1}\right)\right)=k\left(\Phi\left(t, \varphi, \psi^{2}\right)\right) \quad \text { for all } t \geq 0 .
$$

Proof. From theorem 3.4 we know that $\Phi$ exists. Let $\left(x^{i}, y^{i}\right):[-r, \infty) \rightarrow \mathbb{R}^{2}$ be given such that $\left.\Phi\left(t, \varphi^{i}, \psi^{i}\right)=\left(x_{t}^{i}, y_{t}^{i}\right), t \geq 0\right)$.

First we show that

$$
x^{1}(t)=x^{2}(t) \text { for all } t \in[-r, \infty), \quad y^{1}(t)=y^{2}(t) \text { for all } t \in\left[-\zeta-r_{1}, \infty\right) .
$$

If (3.13) does not hold, then there exists a maximal $t_{0} \in[0, \infty)$ such that

$$
x^{1}(t)=x^{2}(t) \text { for all } t \in\left[-r, t_{0}\right], \quad y^{1}(t)=y^{2}(t) \text { for all } t \in\left[-\zeta-r_{1}, t_{0}\right] .
$$

We claim that $\sigma\left(y_{t}^{1}\right)=\sigma\left(y_{t}^{2}\right)$ for all $t \in\left[0, t_{0}+r_{1}\right]$.

proposition 3.8 implies, for $i=1,2$, that

$$
t-\sigma\left(y_{t}^{i}\right)-r_{1} \geq-\sigma\left(y_{0}^{i}\right)-r_{1}=-\zeta-r_{1} \text { for all } t \in[0, \infty) .
$$

From this inequality and from lemma 3.5, it follows for each $t \in\left[0, t_{0}+r_{1}\right]$ that

$$
\begin{aligned}
& \left|\sigma\left(y_{t}^{1}\right)-\sigma\left(y_{t}^{2}\right)\right| \leq \frac{1}{a} \max _{s \in\left[-\max \left\{\sigma\left(y_{t}^{1}\right), \sigma\left(y_{t}^{2}\right)\right\}-r_{1},-r_{1}\right]}\left|y^{1}(t+s)-y^{2}(t+s)\right| \\
& =\frac{1}{a} \max _{s \in\left[t-\max \left\{\sigma\left(y_{t}^{1}\right), \sigma\left(y_{t}^{2}\right)\right\}-r_{1}, t-r_{1}\right]}\left|y^{1}(s)-y^{2}(s)\right| \leq \frac{1}{a} \max _{s \in\left[-\zeta-r_{1}, t_{0}\right]}\left|y^{1}(s)-y^{2}(s)\right|=0 .
\end{aligned}
$$

Therefore the claim holds.

Set $m(t)=\sigma\left(y_{t}^{1}\right)=\sigma\left(y_{t}^{2}\right), t \in\left[0, t_{0}+r_{1}\right]$. Clearly, for both $x^{1}$ and $x^{2}$ the same equation $\dot{x}(t)=G\left(x_{t}, m(t)\right)$ holds for all $t \in\left(0, t_{0}+r_{1}\right)$. By (3.14), $x_{t}^{1}=x_{t}^{2}$ for all $t \in\left[0, t_{0}\right]$. Since $G: X \times Z \rightarrow \mathbb{R}$ is Lipschitz continuous, an application of Gronwall's inequality yields the existence of a $\delta \in\left(0, r_{1}\right)$ so that $x^{1}(t)=x^{2}(t)$ for all $t \in\left[-r, t_{0}+\delta\right]$. Now we can apply lemma 3.2 to conclude $y^{1}(t)=y^{2}(t)$ for all $t \in\left[-\zeta-r_{1}, t_{0}+\delta\right]$. This contradicts the definition of $t_{0}$. It follows that (3.13) is satisfied, and also $k\left(x_{t}^{1}, y_{t}^{1}\right)=k\left(x_{t}^{2}, y_{t}^{2}\right)$ for all $t \geq 0$. This proves our statement.

Now we have all tools to show that for System (1.6), (1.2), (1.3) the space $X \times Z$ is a suitable phase space. 
Theorem 3.10. For each $(\varphi, \zeta) \in X \times Z$ there exists a unique pair of functions

$$
x^{\varphi, \zeta}:[-r, \infty) \rightarrow \mathbb{R}, \quad z^{\varphi, \zeta}:[0, \infty) \rightarrow \mathbb{R}
$$

such that $(x, z)$ is a solution of System (1.6), (1.2), (1.3) in the phase space $X \times Z$ satisfying the initial condition $x_{0}^{\varphi, \zeta}=\varphi, z^{\varphi, \zeta}(0)=\zeta$. The mapping

$$
\Psi:[0, \infty) \times X \times Z \ni(t, \varphi, \zeta) \mapsto\left(x_{t}^{\varphi, \zeta}, z^{\varphi, \zeta}(t)\right) \in X \times Z
$$

defines a continuous semiflow. In addition, there exists a constant $M>0$ such that

$$
\left\|\Psi\left(t, \varphi^{1}, \zeta^{1}\right)-\Psi\left(t, \varphi^{2}, \zeta^{2}\right)\right\| \leq M\left\|\left(\varphi^{1}, \zeta^{1}\right)-\left(\varphi^{2}, \zeta^{2}\right)\right\| e^{t(1+L)}
$$

for all $t \geq 0, \varphi^{1}, \varphi^{2} \in X, \zeta^{1}, \zeta^{2} \in Z$. Moreover, for all $t \geq 0, \varphi \in X, \zeta \in Z$,

$$
\Psi(t, \varphi, \zeta)=k(\Phi(t, h(\varphi, \zeta)))
$$

Proof. Let $(\varphi, \zeta) \in X \times Z$ be given.

1. Existence. By theorem 3.4, System (3.11), (1.2) has a unique solution in the phase space $X \times Y$, denoted by $\left(x^{\varphi, \gamma(\varphi, \zeta)}, y^{\varphi, \gamma(\varphi, \zeta)}\right):[-r, \infty) \rightarrow \mathbb{R}^{2}$, with $x_{0}=\varphi, y_{0}=\gamma(\varphi, \zeta)$. Define $\Psi:[0, \infty) \times X \times Z \rightarrow X \times Z$ by

$$
\Psi(t, \varphi, \zeta)=k(\Phi(t, h(\varphi, \zeta)))=k\left(x_{t}^{\varphi, \gamma(\varphi, \zeta)}, y_{t}^{\varphi, \gamma(\varphi, \zeta)}\right)=\left(x_{t}^{\varphi, \gamma(\varphi, \zeta)}, \sigma\left(y_{t}^{\varphi, \gamma(\varphi, \zeta)}\right)\right)
$$

$\Psi$ is continuous since the functions $h, k$ and $\Phi$ are continuous. The pair

$$
x^{\varphi, \zeta}(t)=x^{\varphi, \gamma(\varphi, \zeta)}(t), \quad t \in[-r, \infty), \quad z^{\varphi, \zeta}(t)=\sigma\left(y_{t}^{\varphi, \gamma(\varphi, \zeta)}\right), \quad t \in[0, \infty),
$$

is a solution of System (1.6), (1.2), (1.3) in $X \times Z$ with $x_{0}=\varphi, z(0)=\zeta$.

2. Uniqueness. Assume the pair of functions $\widetilde{x}:[-r, \omega) \rightarrow \mathbb{R}, \widetilde{z}:[0, \omega) \rightarrow \mathbb{R}$ is also a solution of System (1.6), (1.2), (1.3) in $X \times Z$ with initial condition $\widetilde{x}_{0}=\varphi$, $\widetilde{z}(0)=\zeta$ and $0<\omega \leq \infty$. Then, by definition, there exists a Lipschitz continuous function $\widetilde{y}:[-r, \omega) \rightarrow \mathbb{R}$ so that $\widetilde{y}_{t} \in Y, \widetilde{z}(t)=\sigma\left(\widetilde{y}_{t}\right)$ for all $t \in[0, \omega)$, and equation (1.2) holds a.e. in $\left[-\zeta-r_{1}, \omega\right)$. From $\widetilde{z}(0)=\zeta=\sigma\left(\widetilde{y}_{0}\right)=(1 / c) \widetilde{y}\left(-\zeta-r_{1}\right)$ and (1.2), it easily follows that

$$
\widetilde{y}(s)=\gamma(\varphi, \zeta)(s) \text { for all } s \in\left[-\zeta-r_{1}, 0\right] .
$$

Now proposition 3.9 implies that

$$
\left(x_{t}, z(t)\right)=k(\Phi(t, \varphi, \gamma(\varphi, \zeta)))=k\left(\Phi\left(t, \varphi, \widetilde{y}_{0}\right)\right)=\left(\widetilde{x}_{t}, \widetilde{z}(t)\right) \quad \text { for all } t \in[0, \omega),
$$

and the proof of uniqueness is completed.

3. Properties of $\Psi$. We have to show that $\Psi$ is a semiflow on $X \times Z$, i.e.,

$$
\Psi\left(t_{1}+t_{2}, \varphi, \zeta\right)=\Psi\left(t_{2}, \Psi\left(t_{1}, \varphi, \zeta\right)\right) \text { for all } t_{1} \geq 0, t_{2} \geq 0
$$

Let $x:[-r, \infty) \rightarrow \mathbb{R}, z:[-\zeta-1, \infty) \rightarrow \mathbb{R}$ be the solution of System (1.6), (1.2), (1.3), $y:[-r, \infty) \rightarrow \mathbb{R}$ be such that (1.2) holds a.e. in $[-r, \infty)$, and $t_{1} \geq 0, t_{2} \geq 0$.

Since $\Psi(t, \varphi, \zeta)=\left(x_{t}, z(t)\right)$ for $t \geq 0$, equation (3.16) is equivalent to

$$
\left(x_{t_{1}+t_{2}}, z\left(t_{1}+t_{2}\right)\right)=\Psi\left(t_{2},\left(x_{t_{1}}, z\left(t_{1}\right)\right)\right) .
$$


Using the functions $h$ and $k$ defined in (3.12), it is easy to see that

$$
\Psi(t, \varphi, \zeta)=k(\Phi(t, h(\varphi, \zeta))) \quad \text { for all } t \geq 0,
$$

so equation (3.17) can be written as

$$
\left(x_{t_{1}+t_{2}}, z\left(t_{1}+t_{2}\right)\right)=k\left(\Phi\left(t_{2}, x_{t_{1}}, \gamma\left(x_{t_{1}}, z\left(t_{1}\right)\right)\right)\right) .
$$

The assumptions of proposition 3.9 clearly hold with $x_{t_{1}}, \gamma\left(x_{t_{1}}, z\left(t_{1}\right)\right), y_{t_{1}}, z\left(t_{1}\right)$ instead of $\varphi, \psi^{1}, \psi^{2}, \zeta$, so we have

$$
\begin{aligned}
k\left(\Phi\left(t_{2}, x_{t_{1}}, \gamma\left(x_{t_{1}}, z\left(t_{1}\right)\right)\right)\right) & =k\left(\Phi\left(t_{2}, x_{t_{1}}, y_{t_{1}}\right)\right)=k\left(x_{t_{1}+t_{2}}, y_{t_{1}+t_{2}}\right) \\
& =\left(x_{t_{1}+t_{2}}, \sigma\left(y_{t_{1}+t_{2}}\right)\right)=\left(x_{t_{1}+t_{2}}, z\left(t_{1}+t_{2}\right)\right) .
\end{aligned}
$$

Thus, (3.18) holds, and $\Psi$ is a semiflow on $X \times Z$.

From the Lipschitz property of $\Phi$ in theorem 3.4, and the Lipschitz continuity of $h$ and $k, \Psi$ has the Lipschitz property (3.15) with some $M>0$.

\section{Slowly oscillating periodic solutions}

In this section it is shown that for System (1.8), (1.9), (1.10) it is possible to have slowly oscillatory periodic solutions. The model of Ranjan et al. [26, 25], i.e., System (1.4), (1.2), (1.3) will be a particular case whenever $r_{0}=0, r_{1}=1$ and $U, p$ are suitable, see section 5 .

Recall from section 1 the constants $a, b, c, q$, with $0<a<c<b, q>0$. For the rate $x(t), x(t) \in[a, b]$ is assumed, $c$ is the maximal capacity of the server, $q$ is an upper bound for the length of the queue $y(t)$. We suppose that there exists $x_{*} \in(a, c)$ serving as a stationary solution of the rate control equation.

Set $d=c-x_{*}>0, A=a-x_{*}<0, B=b-x_{*}>d$, and assume the following conditions on $f$ and $g$ :

(S1) $f, g \in C^{1}([A, B], \mathbb{R})$;

(S2) $f(\xi) \xi \geq 0$ and $g(\xi) \xi>0$ for all $\xi \in[A, B] \backslash\{0\}, g^{\prime}(0)>0$;

(S3) $g([A, B]) \in(-f(B),-f(A))$;

(S4) the map $\mathbb{C} \ni \lambda \mapsto \lambda+f^{\prime}(0)+g^{\prime}(0) e^{-\lambda} \in \mathbb{C}$ has a zero with positive real part.

Now we define the system for which we will be able to show the existence of a periodic solution. As we are interested in the oscillatory behaviour of $x(t)$ around $x_{*}$ we introduce $v(t)=x(t)-x_{*}$, and write the rate control equation for $v$ instead of $x$. In the rest of the paper we consider System (1.8), (1.9), (1.10). Note that Equations (1.2), (1.3), with $r_{0}=0, r_{1}=1$ and $x(t)=v(t)+x_{*}$, become Equations (1.9), (1.10), respectively. Moreover, equation (1.8) is a particular case of equation (1.6) provided $r_{0}=0, r_{1}=1$ and $x(t)=v(t)+x_{*}$, and $G: X \times Z \rightarrow \mathbb{R}$ is given by

$$
G(\varphi, \zeta)=-f\left(\varphi(0)-x_{*}\right)-g\left(\varphi(-\zeta-1)-x_{*}\right) .
$$

Define the functions $\tilde{f}, \widetilde{g}:[A, B] \rightarrow \mathbb{R}$ as follows:

$$
\widetilde{f}(\xi)=\left\{\begin{array}{ll}
\frac{f(\xi)}{\xi} & \text { if } \xi \neq 0, \\
f^{\prime}(0) & \text { if } \xi=0,
\end{array} \quad \widetilde{g}(\xi)= \begin{cases}\frac{g(\xi)}{\xi} & \text { if } \xi \neq 0, \\
g^{\prime}(0) & \text { if } \xi=0 .\end{cases}\right.
$$


From (S1) and (S2) it follows that $\widetilde{f}$ and $\widetilde{g}$ are continuous, and there are constants $f_{1} \geq 0$, $g_{1}>g_{0}>0$ such that

$$
\widetilde{f}([A, B]) \subseteq\left[0, f_{1}\right], \quad \widetilde{g}([A, B]) \subseteq\left[g_{0}, g_{1}\right]
$$

Let

$$
r=1+\frac{q}{c}, \quad K_{0}=\left(f_{1}+g_{1}\right) \max \{-A, B\}, \quad K_{1}=r K_{0} .
$$

For $\varphi \in C_{[-r, 0]}$ and $k \in \mathbb{R}$ define $\varphi+k \in C_{[-r, 0]}$ as $[-r, 0] \ni s \mapsto \varphi(s)+k \in \mathbb{R}$.

Under Hypotheses (S1)-(S3), with the above definition of $G$, it is clear that Conditions (G1)-(G3) hold with $L_{G}=\max \left\{\|f\|_{1}+\|g\|_{1}, K\|g\|_{1}\right\}, K=K_{1}$, where $\|f\|_{1}=$ $\max _{\xi \in[A, B]}\left|f^{\prime}(\xi)\right|,\|g\|_{1}=\max _{\xi \in[A, B]}\left|g^{\prime}(\xi)\right|$. Therefore, by theorem 3.10, for all $(\varphi, \zeta) \in$ $X \times Z$, there exists a unique solution $x^{\varphi, \zeta}:[-r, \infty) \rightarrow \mathbb{R}, z^{\varphi, \zeta}:[0, \infty) \rightarrow \mathbb{R}$ with $x_{0}^{\varphi, \zeta}=\varphi$, $z^{\varphi, \zeta}(0)=\zeta$, and $\Psi(t, \varphi, \zeta)=\left(x_{t}^{\varphi, \zeta}, z^{\varphi, \zeta}(t)\right)$. In addition, there exists a unique function $y^{\varphi, \zeta}:[-r, \infty) \rightarrow \mathbb{R}$ with $y_{t}^{\varphi, \zeta} \in Y$ for all $t \geq 0, y_{0}^{\varphi, \zeta}=\gamma(\varphi, \zeta)$ such that equation (1.2) with $r_{0}=0, x(t)=x^{\varphi, \zeta}(t), y(t)=y^{\varphi, \zeta}(t)$ holds a.e. in $[-\zeta-1, \infty)$, and $z^{\varphi, \zeta}(t)=\sigma\left(y_{t}^{\varphi, \zeta}\right)$ for all $t \geq 0$. Introduce the set

$$
\mathcal{X}=\left\{\varphi \in C_{[-r, 0]} \mid \varphi([-r, 0]) \subseteq[A, B], \operatorname{lip}(\varphi) \leq K_{1}\right\} .
$$

By $K=K_{1}$, we have

$$
\mathcal{X}=\left\{\varphi \in C_{[-r, 0]} \mid \varphi+x_{*} \in X\right\} .
$$

Observe that, for all $(\varphi, \zeta) \in X \times Z$, the functions $v:[-r, \infty) \rightarrow \mathbb{R}, y:[-r, \infty) \rightarrow \mathbb{R}$, $z:[0, \infty) \rightarrow \mathbb{R}$ given by $v(t)=x^{\varphi, \zeta}(t)-x_{*}, y(t)=y^{\varphi, \zeta}(t), z(t)=z^{\varphi, \zeta}(t)$ are solutions of System (1.8), (1.9), (1.10) in the sense that (1.8) holds for all $t>0,(1.9)$ is satisfied a.e. in $[-\zeta-1, \infty),(1.10)$ is valid for all $t \geq 0$, and $v_{0}=\varphi-x_{*} \in \mathcal{X}, z(0)=\zeta$. Note that only $x^{\varphi, \zeta}$ is shifted by $x_{*}, y^{\varphi, \zeta}$ and $z^{\varphi, \zeta}$ are unchanged to get solutions of (1.8), (1.9), (1.10) from that of (1.6), (1.2), (1.3). Therefore, theorem 3.10 for (1.6), (1.2), (1.3) in the above specified case immediately gives existence and uniqueness of solutions for (1.8), (1.9), (1.10). Moreover, it is natural to use $v^{\varphi, \zeta}, z^{\varphi, \zeta}, y^{\varphi, \zeta}$ for the unique solution of (1.8), (1.9), (1.10) as well, where $(\varphi, \zeta) \in \mathcal{X} \times Z$. Now, for each $(\varphi, \zeta) \in \mathcal{X} \times Z$, the unique solution $v=v^{\varphi, \zeta}:[-r, \infty) \rightarrow \mathbb{R}, z=z^{\varphi, \zeta}:[0, \infty) \rightarrow \mathbb{R}$ of System (1.8), (1.9), (1.10) with $v_{0}=\varphi, z(0)=\zeta$ can be determined as

$$
\left(v_{t}^{\varphi, \zeta}, z^{\varphi, \zeta}(t)\right)=\Psi\left(t, \varphi+x_{*}, \zeta\right)-\left(x_{*}, 0\right)
$$

and we obtain

Proposition 4.1. Under Conditions (S1)-(S3), the solutions of System (1.8), (1.9), (1.10) define the continuous semiflow

$$
\Theta:[0, \infty) \times \mathcal{X} \times Z \ni(t, \varphi, \zeta) \mapsto\left(v_{t}^{\varphi, \zeta}, z^{\varphi, \zeta}(t)\right) \in \mathcal{X} \times Z
$$

Moreover,

$$
\left\|\Theta\left(t, \varphi^{1}, \zeta^{1}\right)-\Theta\left(t, \varphi^{2}, \zeta^{2}\right)\right\| \leq M\left\|\left(\varphi^{1}, \zeta^{1}\right)-\left(\varphi^{2}, \zeta^{2}\right)\right\| e^{t(1+L)}
$$

for all $t \geq 0, \varphi^{1}, \varphi^{2} \in \mathcal{X}, \zeta^{1}, \zeta^{2} \in Z$, with the same constants $M>0, L>0$ as given in theorem 3.10 provided $L_{G} \leq L \min \{1, a\}$. 
In addition to $v^{\varphi, \zeta}$ and $z^{\varphi, \zeta}$, there exists a unique $y=y^{\varphi, \zeta}:[-r, \infty) \rightarrow \mathbb{R}$ with $y_{t} \in Y$ for all $t \geq 0, y_{0}=\gamma(\varphi, \zeta)$, (1.10) holds for all $t \geq 0$, and (1.9) holds almost everywhere on $[-\zeta-1, \infty)$.

In the sequel, when we write $(v, z, y)$, we always mean that $v=v^{\varphi, \zeta}, z=z^{\varphi, \zeta}, y=y^{\varphi, \zeta}$ for some $(\varphi, \zeta) \in \mathcal{X} \times Z$. Recall also the function $\eta=\eta^{\varphi, \zeta}:[0, \infty) \ni t \mapsto t-z^{\varphi, \zeta}(t)-1 \in \mathbb{R}$ and $\eta^{-1}=\left(\eta^{\varphi, \zeta}\right)^{-1}:[-\zeta-1, \infty) \rightarrow \mathbb{R}$ and their properties from proposition 3.8:

$$
\operatorname{slope}(\eta) \subseteq[c / b, c / a], \quad \operatorname{slope}\left(\eta^{-1}\right) \subseteq[a / c, b / c] .
$$

In particular $\eta$ and $\eta^{-1}$ are increasing functions with $t-r \leq \eta(t) \leq t-1$ for all $t \geq 0$, and $t+1 \leq \eta^{-1}(t) \leq t+r$ for all $t \geq-\zeta-1$.

Define $T_{0}=2 q / d$.

Proposition 4.2. If $\tau_{1} \geq-\zeta-1, \tau_{2} \geq \tau_{1}+T_{0}$, and $v(t) \leq d / 2$ for all $t \in\left[\tau_{1}, \tau_{2}\right]$, then $y(t)=0$ for all $t \in\left[\tau_{1}+T_{0}, \tau_{2}\right]$. If, in addition, $\tau_{2} \geq \tau_{1}+T_{0}+1$, then $z(t)=0$ for all $t \in\left[\tau_{1}+T_{0}+1, \tau_{2}\right]$.

Proof. From equation (1.9) and from $v(t) \leq d / 2, t \in\left[\tau_{1}, \tau_{2}\right]$, it follows that, if there is $\tau_{*} \in\left[\tau_{1}, \tau_{2}\right)$ with $y\left(\tau_{*}\right)=0$, then $\dot{y}(t) \leq 0$ almost everywhere in $\left[\tau_{*}, \tau_{2}\right]$, and thus, $y(t)=0$ for all $t \in\left[\tau_{*}, \tau_{2}\right]$. Consequently, either $y(t)=0$ for all $t \in\left[\tau_{1}, \tau_{2}\right]$, or there exists a maximal $\tau_{* *} \in\left(\tau_{1}, \tau_{2}\right]$ with $y(t)>0$ for all $t \in\left[\tau_{1}, \tau_{* *}\right)$. In the first case the statements of the proposition trivially hold. In the second case, by equation (1.9), $\dot{y}(t)=$ $v(t)-d \leq-d / 2$ almost everywhere in $\left[\tau_{1}, \tau_{* *}\right]$. As $y\left(\tau_{1}\right) \in[0, q]$, it easily follows that $0 \leq y\left(\tau_{* *}\right) \leq q-(d / 2)\left(\tau_{* *}-\tau_{1}\right)$, and hence $\tau_{* *} \leq \tau_{1}+T_{0}$. Therefore, $y(t)=0$ for all $t \in\left[\tau_{1}+T_{0}, \tau_{2}\right]$. The statement for $z$ can be obtained by using equation (1.10).

Observe that $(0,0) \in \mathcal{X} \times Z$ is a stationary point of the semiflow $\Theta$ generated by System (1.8), (1.9), (1.10). Under Conditions (S1)-(S3), and assuming that (S4) does not hold, and slightly more, that is

(S5) $\operatorname{Re} z<0$ for all zeros of the map $\mathbb{C} \ni \lambda \mapsto \lambda+f^{\prime}(0)+g^{\prime}(0) e^{-\lambda} \in \mathbb{C}$,

it is expected that $(0,0)$ is stable. In fact, combining propositions 4.1 and 4.2 , local stability is straightforward.

Theorem 4.3. Assume that Conditions (S1)-(S3), (S5) hold. Then the stationary point $(0,0) \in \mathcal{X} \times Z$ of the semiflow $\Theta$ generated by System (1.8), (1.9), (1.10) is locally asymptotically stable.

Proof. By proposition 4.1, for each $(\varphi, \zeta) \in \mathcal{X} \times Z$ the unique solution $v=v^{\varphi, \zeta}, z=z^{\varphi, \zeta}$ of System (1.8), (1.9), (1.10) satisfies

$$
\begin{aligned}
\left\|\left(v_{t}, z(t)\right)-(0,0)\right\| & =\|\Theta(t, \varphi, \zeta)-\Theta(t, 0,0)\| \\
& \leq M\|(\varphi, \zeta)\| e^{t(1+L)} \leq M\|(\varphi, \zeta)\| e^{\left(T_{0}+1\right)(1+L)}
\end{aligned}
$$

for all $t \in\left[0, T_{0}+1\right]$.

As proposition 4.2 holds with $\tau_{1}=-1$ and arbitrarily large $\tau_{2}$, if $v(t) \leq d / 2$ for all $t \geq-r$, then $z(t)=0$ for all $t \geq-1+T_{0}+1=T_{0}$, and, consequently,

$$
\dot{v}(t)=-f(v(t))-g(v(t-1)) \quad \text { for all } t>T_{0}+1 .
$$


A classical result for equations with constant delay (see e.g. [7, 9]) is that, under Conditions (S1)-(S3), (S5), for each $\varepsilon \in(0, d / 2)$ there exists $\delta=\delta(\varepsilon) \in(0, \varepsilon)$ with

$$
\max _{-1 \leq s \leq 0}\left|v\left(T_{0}+1+s\right)\right| \leq \delta \text { implies }|v(t)|<\varepsilon \text { for all } t \geq T_{0} .
$$

For given $\varepsilon \in(0, d / 2)$ choosing $(\varphi, \zeta) \in \mathcal{X} \times Z$ with $\|(\varphi, \zeta)\|<\varepsilon e^{-\left(T_{0}+1\right)(1+L)} / M$, it should be clear that $\left\|\left(v_{t}, z(t)\right)\right\|<\varepsilon$ follows for all $t \geq 0$. That is, $(0,0)$ is locally stable.

Asymptotic stability follows in the same way by using again the constant delay result from $[7]$.

From this point throughout this section, we assume that Conditions (S1)-(S4) are satisfied. Then instability of $(0,0) \in \mathcal{X} \times Z$ can be easily obtained. We show after a series of technical results that there exists a nontrivial slowly oscillating periodic solution $(v, z)$ of System (1.8), (1.9), (1.10). Here slow oscillation of $(v, z)$ means that

$$
t_{1}<t_{2}-z\left(t_{2}\right)-1
$$

holds for any two zeros $t_{1}<t_{2}$ of $v$.

Observe that equation (1.8) can be written as

$$
\dot{v}(t)=-\tilde{f}(v(t)) v(t)-\widetilde{g}(v(t-z(t)-1)) v(t-z(t)-1) .
$$

For $(\varphi, \zeta) \in \mathcal{X} \times Z$ consider $v=v^{\varphi, \zeta}, z=z^{\varphi, \zeta}$. Define

$$
\begin{aligned}
& u=u^{\varphi, \zeta}:[-r, \infty) \ni t \mapsto v(t) \exp \left(\int_{0}^{t} \widetilde{f}(v(s)) d s\right) \in \mathbb{R} \quad \text { and } \\
& C=C^{\varphi, \zeta}:[0, \infty) \ni t \mapsto \widetilde{g}(v(t-z(t)-1)) \exp \left(\int_{t-z(t)-1}^{t} \tilde{f}(v(s)) d s\right) \in \mathbb{R} .
\end{aligned}
$$

Setting $c_{0}=g_{0}, c_{1}=g_{1} e^{f_{1} r}$, for all $(\varphi, \zeta) \in \mathcal{X} \times Z$ we have

$$
C(t) \in\left[c_{0}, c_{1}\right] \text { for all } t \geq 0 .
$$

Proposition 4.4. For each $(\varphi, \zeta) \in \mathcal{X} \times Z$, the functions $v=v^{\varphi, \zeta}, z=z^{\varphi, \zeta} u=u^{\varphi, \zeta}$ $C=C^{\varphi, \zeta} R$ are continuous, $u$ is continuously differentiable on $(0, \infty)$, and

$$
\dot{u}(t)=-C(t) u(t-z(t)-1) \quad(t>0)
$$

holds. In addition,

$$
\begin{aligned}
& |u(t)| \leq|v(t)| \leq|u(t)| e^{f_{1} r} \quad \text { for all } t \in[-r, 0], \\
& |v(t)| \leq|u(t)| \leq|v(t)| e^{f_{1} t} \quad \text { for all } t \geq 0 .
\end{aligned}
$$

Proof. The continuity and differentiability properties are immediate from the definitions. Differentiating $u$ and using equation (4.3) for $t>0$, we get

$$
\begin{aligned}
\dot{u}(t) & =(\dot{v}(t)+\widetilde{f}(v(t)) v(t)) \exp \left(\int_{0}^{t} \widetilde{f}(v(s)) d s\right) \\
& =-\widetilde{g}(v(t-z(t)-1)) v(t-z(t)-1) \\
& \cdot \exp \left(\int_{0}^{t-z(t)-1} \tilde{f}(v(s)) d s\right) \exp \left(\int_{t-z(t)-1}^{t} \tilde{f}(v(s)) d s\right) \\
& =-C(t) u(t-z(t)-1),
\end{aligned}
$$

so equation (4.4) holds. The stated inequalities between $|u(t)|$ and $|v(t)|$ are easy consequences of the definitions and the bounds on $\widetilde{f}$. 
Let

$$
\begin{aligned}
W=\{(\varphi, \zeta) \in \mathcal{X} \times Z \mid & \varphi(s)=0 \text { for all } s \in[-r,-1-\zeta], \\
& {\left.[-\zeta-1,0] \ni s \mapsto \varphi(s) e^{f_{1} s} \in \mathbb{R} \text { is nondecreasing, } \varphi(0)>0\right\} }
\end{aligned}
$$

and $W_{0}=W \cup\{(0,0)\}$. Our plan is to define a return map on $W_{0}$ and to show that it has a nontrivial fixed point on $W_{0}$ corresponding to a slowly oscillating periodic orbit.

Proposition 4.5. There exists a constant $T_{2}>1$ such that for all $(\varphi, \zeta) \in W, v=v^{\varphi, \zeta}$ has at least two zeros in $\left[0, T_{2}\right]$. More precisely, there exist $t_{2}>t_{1}>0$ with $t_{2} \leq T_{2}$, $v(t)>0$ on $\left[0, t_{1}\right) \cup\left(t_{2}, \eta^{-1}\left(t_{2}\right)\right], v\left(t_{1}\right)=0, v(t)<0$ on $\left(t_{1}, t_{2}\right), v\left(t_{2}\right)=0$, and $\dot{v}\left(t_{1}\right)<0$, $\dot{v}\left(t_{2}\right)>0$. In addition, the function $u=u^{\varphi, \zeta}$ is nonnegative on $[-r, 0]$, it is positive on $\left[0, t_{1}\right) \cup\left(t_{2}, \eta^{-1}\left(t_{2}\right)\right]$, negative on $\left(t_{1}, t_{2}\right)$, it is nonincreasing on $\left[0, \eta^{-1}\left(t_{1}\right)\right]$, and increasing on $\left[\eta^{-1}\left(t_{1}\right), \eta^{-1}\left(t_{2}\right)\right]$.

Proof. As $v=v^{\varphi, \zeta}$ and $u=u^{\varphi, \zeta}$ have the same zeros, it suffices to show the statement for $u=u^{\varphi, \zeta}$.

Let $\lambda$ be a zero of $\lambda \mapsto \lambda+f^{\prime}(0)+g^{\prime}(0) e^{-\lambda}$ with Re $\lambda>0$ guaranteed by hypothesis (S4). Setting $\mu=\lambda+f^{\prime}(0)$, we have $\operatorname{Re} \mu>0$ and $\mu+g^{\prime}(0) e^{f^{\prime}(0)} e^{-\mu}=0$. This is possible only if $g^{\prime}(0) e^{f^{\prime}(0)}>\pi / 2\left(\right.$ see $\left[7\right.$, Ch. XI.]). As $\widetilde{f}, \widetilde{g}$ are continuous and $\tilde{f}(0)=f^{\prime}(0), \widetilde{g}(0)=g^{\prime}(0)$, there exists $\delta \in(0, d / 2)$ such that

$$
\widetilde{g}\left(\xi_{1}\right) e^{\widetilde{f}\left(\xi_{2}\right)}>\frac{\pi}{2} \quad \text { for }\left|\xi_{1}\right| \leq \delta,\left|\xi_{2}\right| \leq \delta .
$$

Observe that $B / \delta>1$. Define

$$
s_{0}=r+\frac{1}{c_{0}} \log \frac{B e^{f_{1} r}}{\delta}, \quad s_{1}=s_{0}+T_{0}+1, \quad T_{1}=s_{1}+7 .
$$

First, we prove that for all $(\varphi, \zeta) \in W, u=u^{\varphi, \zeta}$ has at least one zero in $\left[0, T_{1}\right]$. Indirectly, assume that there exists a $(\varphi, \zeta) \in W$ such that $u(t)>0$ for all $t \in\left[0, T_{1}\right]$. By the definition of $W$ and our assumption, $u$ is nonnegative on $\left[-r, T_{1}\right]$. From proposition 4.4 and equation (4.4) it follows that $\dot{u}(t) \leq 0$ for all $t \in\left(0, T_{1}\right]$. Thus, $u$ is monotone nonincreasing on $\left[0, T_{1}\right]$. In particular, $u(t) \leq u(t-z(t)-1)$ for $t \in\left[r, T_{1}\right]$. Then, again by proposition 4.4 ,

$$
\dot{u}(t) \leq-c_{0} u(t) \quad \text { for all } t \in\left[r, T_{1}\right] .
$$

From $v(r) \leq B, u(r) \leq B e^{f_{1} r}$, inequality (4.6), $B e^{f_{1} r} e^{-c_{0}\left(s_{0}-r\right)}=\delta, s_{0}>r$, we get

$$
v(t) \leq u(t) \leq B e^{f_{1} r} e^{-c_{0}(t-r)} \leq B e^{f_{1} r} e^{-c_{0}\left(s_{0}-r\right)}=\delta<\frac{d}{2} \quad \text { for all } \in\left[s_{0}, T_{1}\right]
$$

Applying proposition 4.2 with $\tau_{1}=s_{0}, \tau_{2}=T_{1}$, we find $z(t)=0$ for all $t \in\left[s_{1}, T_{1}\right]$. This means that equation (4.4) becomes

$$
\dot{u}(t)=-C(t) u(t-1) \quad \text { for all } t \in\left[s_{1}, T_{1}\right]
$$

where, by $v(t) \leq \delta$ for all $t \in\left[s_{0}, T_{1}\right]$, and by the choice of $\delta$,

$$
C(t)=\widetilde{g}(v(t-1)) \exp \left(\int_{t-1}^{t} \widetilde{f}(v(s)) d s\right) \geq \widetilde{g}(v(t-1)) \exp \left(\min _{s \in[t-1, t]} \widetilde{f}(v(s))\right)>\frac{\pi}{2} .
$$


There exists a minimal integer $N \geq 1$ with $4 N \geq s_{1}+1$. Clearly, $4 N \leq s_{1}+$ 5 and $4 N+2 \leq T_{1}=s_{1}+7$. The function $t \mapsto \sin ((\pi / 2) t)$ is positive on $(4 N, 4 N+2)$, has zeros at $4 N$ and $4 N+2$. Define

$$
w_{\varepsilon}(t)=\varepsilon \sin \left(\frac{\pi}{2} t\right), \quad \varepsilon>0, t \in \mathbb{R} .
$$

As $u$ is positive on $[4 N, 4 N+2]$, there are a maximal $\varepsilon=\varepsilon_{0}>0$ such that $w(t)=$ $w_{\varepsilon_{0}}(t) \leq u(t)$ for all $t \in[4 N, 4 N+2]$, and a minimal $t \in(4 N, 4 N+2)$, denoted by $\hat{t}$ with $w(\hat{t})=u(\hat{t})$. Now it is clear that

$$
\dot{w}(\hat{t})=\dot{u}(\hat{t}), \quad \text { and } \quad w(t)<u(t) \text { for all } t \in[4 N, \hat{t}) .
$$

From the monotonicity of $u$ on $\left[0, T_{1}\right]$, it follows that $\dot{w}(\hat{t})=\dot{u}(\hat{t}) \leq 0$. Consequently, $\hat{t} \in[4 N+1,4 N+2)$ and $\hat{t}-1 \in[4 N, 4 N+1)$. Hence we obtain $0 \leq w(\hat{t}-1)<u(\hat{t}-1)$.

Therefore, by using $C(\hat{t})>\pi / 2, \dot{w}(t)=-(\pi / 2) w(t-1)$ and $0 \leq w(\hat{t}-1)<u(\hat{t}-1)$, we get

$$
\dot{u}(\hat{t})=-C(\hat{t}) u(\hat{t}-1)<-\frac{\pi}{2} w(\hat{t}-1)=\dot{w}(\hat{t}),
$$

a contradiction to $\dot{u}(\hat{t})=\dot{w}(\hat{t})$. Thus, $u$ has a zero $t_{1}$ in $\left[0, T_{1}\right]$. We may assume that $t_{1}$ is the minimal zero in $\left[0, T_{1}\right]$.

Observe that $(\varphi, \zeta) \in W$ and the definition of $u$ imply the existence of an $s^{*} \in$ $[-\zeta-1,0)$ such that $u(s)=0$ for $s \in\left[-r, s^{*}\right]$, and $u(s)>0$ for $s \in\left(s^{*}, 0\right]$. Then it follows that $u$ is constant on $\left[0, \eta^{-1}\left(s^{*}\right)\right]$, and $\dot{u}(t)<0$ for all $t \in\left(\eta^{-1}\left(s^{*}\right), \eta^{-1}\left(t_{1}\right)\right)$. In particular $\dot{u}\left(t_{1}\right)<0$. Then from inequality (4.5) we conclude that $t_{1}$ is the first zero of $v$ in $[0, \infty)$ and $\dot{v}\left(t_{1}\right)<0$.

From $\dot{u}(t)<0, t \in\left(\eta^{-1}\left(s^{*}\right), \eta^{-1}\left(t_{1}\right)\right), u\left(t_{1}\right)=0, \eta^{-1}\left(s^{*}\right)<t_{1}$, one finds $u(t)<0$ for $t \in\left(t_{1}, \eta^{-1}\left(t_{1}\right)\right]$. Since equation (4.4) is linear in $u$, a similar argument shows that $v$ has a second zero $t_{2}$ in $\left(t_{1}, t_{1}+s_{2}+7\right)$ for some $s_{2}>0$, and $\dot{v}\left(t_{2}\right)>0$.

Let $(\varphi, \zeta) \in W, v=v^{\varphi, \zeta}, z=z^{\varphi, \zeta}, u=u^{\varphi, \zeta}$. Proposition 4.5 allows us to define $t_{0}, t_{1}, t_{2} \in\left[-r, T_{2}\right]$ and $t_{0}^{*}, t_{1}^{*}, t_{2}^{*}$ as

$$
\begin{array}{lll}
t_{0}=t_{0}(\zeta)=-\zeta-1, & t_{0}^{*}=\eta^{-1}\left(t_{0}\right)=0, \\
t_{1}=t_{1}(\varphi, \zeta)=\min \{t>0 \mid v(t)=0\}, & t_{1}^{*}=t_{1}^{*}(\varphi, \zeta)=\eta^{-1}\left(t_{1}\right), \\
t_{2}=t_{2}(\varphi, \zeta)=\min \left\{t>t_{1} \mid v(t)=0\right\}, & t_{2}^{*}=t_{2}^{*}(\varphi, \zeta)=\eta^{-1}\left(t_{2}\right) .
\end{array}
$$

By proposition 4.5, it also follows that

$$
-r \leq t_{0}=-\zeta-1<t_{0}^{*}=0<t_{1}<t_{1}+1 \leq t_{1}^{*}<t_{2}<t_{2}^{*} \leq T_{2}+r .
$$

The continuity of the functions $(\varphi, \zeta) \mapsto t_{j}(\varphi, \zeta)$ plays an important role in the sequel.

Proposition 4.6. The functions

$$
W \ni(\varphi, \zeta) \mapsto t_{j}(\varphi, \zeta) \in\left[-r, T_{2}\right], \quad W \ni(\varphi, \zeta) \mapsto t_{j}^{*}(\varphi, \zeta) \in\left[0, T_{2}+r\right]
$$

are continuous for $j \in\{0,1,2\}$.

Proof. The continuous dependence of $t_{0}$ on the initial functions is evident. Let $(\varphi, \zeta) \in W$ and a sequence $\left(\varphi^{n}, \zeta^{n}\right)_{n=0}^{\infty}$ in $W$ be given with $\left(\varphi^{n}, \zeta^{n}\right) \rightarrow(\varphi, \zeta)$ as $n \rightarrow \infty$ in the norm 
of $C_{[-r, 0]} \times \mathbb{R}$. Proposition 4.1 implies, with the notation $v=v^{\varphi, \zeta}, z=z^{\varphi, \zeta}, v^{n}=v^{\varphi^{n}, \zeta^{n}}$, $z^{n}=z^{\varphi^{n}, \zeta^{n}}$, that

$$
\begin{aligned}
& v^{n}(t) \rightarrow v(t) \text { as } n \rightarrow \infty \text { uniformly in } t \in\left[-r, T_{2}+r\right] \\
& z^{n}(t) \rightarrow z(t) \text { as } n \rightarrow \infty \text { uniformly in } t \in\left[0, T_{2}+r\right] .
\end{aligned}
$$

Then the right hand side of equation (1.8) with $v=v^{n}, z=z^{n}$ tends to the right hand side of (1.8) as $n \rightarrow \infty$ uniformly in $t \in\left[0, T_{2}+r\right]$. Consequently,

$$
\dot{v}^{n}(t) \rightarrow \dot{v}(t) \text { as } n \rightarrow \infty \text { uniformly in } t \in\left(0, T_{2}+r\right] .
$$

It is elementary to show that these uniform convergences guarantee the continuity of $t_{1}(\varphi, \zeta)$ and $t_{2}(\varphi, \zeta)$ in $(\varphi, \zeta)$ since $t_{1}$ and $t_{2}$ are simple zeros. Therefore, $t_{1}(\varphi, \zeta)$ and $t_{2}(\varphi, \zeta)$ are continuous in $(\varphi, \zeta) \in W$.

It also follows from (4.8) that

$$
\eta^{n}(t) \rightarrow \eta(t) \text { as } n \rightarrow \infty \text { uniformly in } t \in\left[0, T_{2}+r\right]
$$

where $\eta=\eta^{\varphi, \zeta}, \eta^{n}=\eta^{\varphi^{n}, \zeta^{n}}$. Define $t_{1}^{n}=t_{1}\left(\varphi^{n}, \zeta^{n}\right)$ and $t_{1}^{n, *}=\left(\eta^{n}\right)^{-1}\left(t_{1}^{n}\right)$. From $t_{1}=\eta\left(t_{1}^{*}\right), t_{1}^{n}=\eta^{n}\left(t_{1}^{n, *}\right)$ and the Lipschitz property of $\eta$ in (4.2), one obtains

$$
\begin{aligned}
\left|t_{1}-t_{1}^{n}\right| & =\left|\eta\left(t_{1}^{*}\right)-\eta^{n}\left(t_{1}^{n, *}\right)\right| \geq\left|\eta\left(t_{1}^{*}\right)-\eta\left(t_{1}^{n, *}\right)\right|-\left|\eta\left(t_{1}^{n, *}\right)-\eta^{n}\left(t_{1}^{n, *}\right)\right| \\
& \geq \frac{c}{b}\left|t_{1}^{*}-t_{1}^{n, *}\right|-\left\|\eta-\eta^{n}\right\|_{\left[0, T_{2}+r\right]}
\end{aligned}
$$

Hence

$$
\left|t_{1}^{*}-t_{1}^{n, *}\right| \leq \frac{b}{c}\left(\left|t_{1}-t_{1}^{n}\right|+\left\|\eta-\eta^{n}\right\|_{\left[0, T_{2}+r\right]}\right) .
$$

This shows $t_{1}^{n, *} \rightarrow t_{1}^{*}, n \rightarrow \infty$, since $t_{1}^{n} \rightarrow t_{1}$ by the first part of the proof, and $\| \eta-$ $\eta^{n} \|_{\left[0, T_{2}+r\right]} \rightarrow 0$ by $(4.9)$.

The proof for $t_{2}^{n, *} \rightarrow t_{2}^{*}$ is analogous.

Define the map $\Gamma: \mathcal{X} \times Z \rightarrow \mathcal{X} \times Z$ by $\Gamma(\varphi, \zeta)=(\widehat{\varphi}, \zeta)$, where $\widehat{\varphi}(s)=\varphi(s)$ for $s \in[-\zeta-1,0]$, and $\widehat{\varphi}(s)=\varphi(-\zeta-1)$ for $s \in[-r,-\zeta-1]$. Clearly, $\Gamma$ is continuous, and $\|\Gamma(\varphi, \zeta)\| \leq\|(\varphi, \zeta)\|$. The existence of $t_{2}^{*}$ allows us to define a return map $P: W_{0} \rightarrow \mathcal{X} \times Z$ by

$$
P(\varphi, \zeta)= \begin{cases}(0,0) & \text { if }(\varphi, \zeta)=(0,0) \\ \Gamma\left(\Theta\left(t_{2}^{*}, \varphi, \zeta\right)\right) & \text { otherwise }\end{cases}
$$

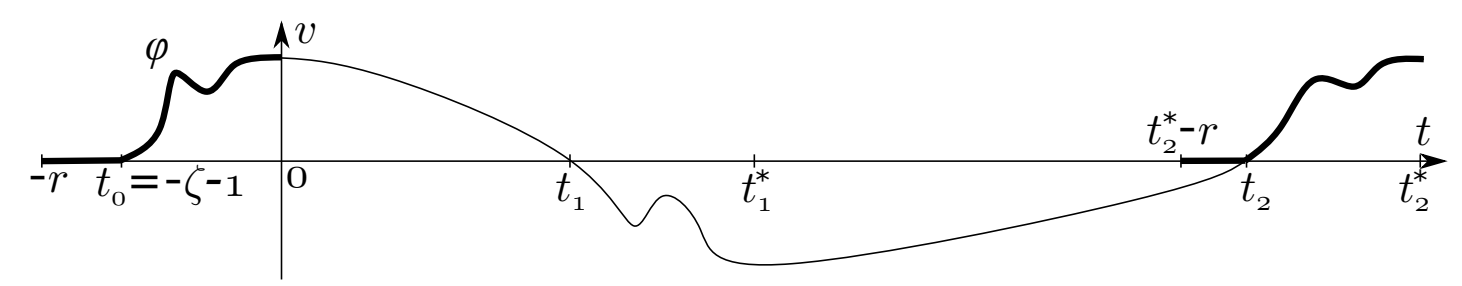

Figure 2: The first component of the return map $P$.

Proposition 4.7. $P$ is continuous, and $P\left(W_{0}\right) \subseteq W_{0}, P(W) \subseteq W$. 
Proof. $P(0,0)=(0,0) \in W_{0}$ trivially. Let $(\varphi, \zeta) \in W$ and $v=v^{\varphi, \zeta}, z=z^{\varphi, \zeta}$.

First we prove $P(\varphi, \zeta)=\Gamma\left(\Theta\left(t_{2}^{*}, \varphi, \zeta\right)\right) \in W$. It is obvious that $\Gamma\left(\Theta\left(t_{2}^{*}, \varphi, \zeta\right)\right) \in \mathcal{X} \times Z$. As $-z\left(t_{2}^{*}\right)-1=t_{2}-t_{2}^{*}$, it remains to show that

$$
\left[-z\left(t_{2}^{*}\right)-1,0\right] \ni s \mapsto v\left(t_{2}^{*}+s\right) e^{f_{1} s} \in \mathbb{R} \text { is monotone nondecreasing. }
$$

If $s \in\left(-z\left(t_{2}^{*}\right)-1,0\right]$ then $v\left(t_{2}^{*}+s\right)>0, v\left(\eta\left(t_{2}^{*}+s\right)\right)<0$, and

$$
\begin{aligned}
& \frac{d}{d s}\left(v\left(t_{2}^{*}+s\right) e^{f_{1} s}\right)=\dot{v}\left(t_{2}^{*}+s\right) e^{f_{1} s}+f_{1} v\left(t_{2}^{*}+s\right) e^{f_{1} s} \\
& =e^{f_{1} s}\left[\left(f_{1}-\widetilde{f}\left(v\left(t_{2}^{*}+s\right)\right)\right) v\left(t_{2}^{*}+s\right)-\widetilde{g}\left(v\left(\eta\left(t_{2}^{*}+s\right)\right)\right) v\left(\eta\left(t_{2}^{*}+s\right)\right)\right]>0 .
\end{aligned}
$$

Thus, $P(\varphi, \zeta)=\Gamma\left(\Theta\left(t_{2}^{*}, \varphi, \zeta\right)\right) \in W$ whenever $(\varphi, \zeta) \in W$.

The continuity of $P$ at $(\varphi, \zeta) \in W$ follows from propositions 4.6 and 4.1.

Continuity of $P$ at $(0,0) \in W_{0}$ is an easy consequence of proposition 4.1 since for $(\varphi, \zeta) \in W$ and $t_{2}^{*}=t_{2}^{*}(\varphi, \zeta)$ we have

$$
\begin{aligned}
\|P(\varphi, \zeta)-P(0,0)\| & =\left\|\Gamma\left(\Theta\left(t_{2}^{*}(\varphi, \zeta), \varphi, \zeta\right)\right)\right\| \leq\left\|\Theta\left(t_{2}^{*}(\varphi, \zeta), \varphi, \zeta\right)\right\| \\
& =\left\|\Theta\left(t_{2}^{*}(\varphi, \zeta), \varphi, \zeta\right)-\Theta\left(t_{2}^{*}(\varphi, \zeta), 0,0\right)\right\| \\
& \leq M\|(\varphi, \zeta)\| e^{t_{2}^{*}(1+L)} \leq M\|(\varphi, \zeta)\| e^{\left(T_{2}+r\right)(1+L)} .
\end{aligned}
$$

Let $(\varphi, \zeta) \in \mathcal{X} \times Z$ and $u=u^{\varphi, \zeta}$. Combining the definitions of $u, \mathcal{X}, f_{1}$, using equation (4.4) and applying proposition 4.4 we obtain

$$
\begin{aligned}
\operatorname{lip}\left(\left.u\right|_{\left[-r, T_{2}+r\right]}\right) & \leq \max \left\{\operatorname{lip}\left(\left.u\right|_{[-r, 0]}\right), \operatorname{lip}\left(\left.u\right|_{\left[0, T_{2}+r\right]}\right)\right\} \\
& \leq \max \left\{\operatorname{lip}\left(v_{0}\right)+\left\|v_{0}\right\|_{[-r, 0]} f_{1}, c_{1}\|u\|_{\left[-r, T_{2}+r\right]}\right\} \\
& \leq \max \left\{K_{1}+f_{1} \max \{-A, B\}, c_{1} e^{f_{1}\left(T_{2}+r\right)} \max \{-A, B\}\right\} \\
& \leq K_{1}+\left(1+c_{1}\right) e^{f_{1}\left(T_{2}+r\right)} \max \{-A, B\} .
\end{aligned}
$$

Choose $L_{1}>0$ such that

$$
L_{1} \geq K_{1}+\left(1+c_{1}\right) e^{f_{1}\left(T_{2}+r\right)} \max \{-A, B\} \quad \text { and } \quad \frac{2 c L_{1}}{c_{0} a} \geq \max \{1,-A, B\} .
$$

Then, clearly, $\operatorname{lip}\left(\left.u\right|_{\left[-r, T_{2}+r\right]}\right) \leq L_{1}$. Define

$$
\beta=\frac{2 c L_{1}}{c_{0} a}, \quad \rho=2^{T_{2}+r} \quad \text { and } \quad \theta=\beta^{-2 \rho} .
$$

Proposition 4.8. For all $(\varphi, \zeta) \in W$,

$$
v\left(t_{2}^{*}\right) \geq \theta(\varphi(0))^{\rho} .
$$

Proof. Let $(\varphi, \zeta) \in W, u=u^{\varphi, \zeta}, \eta=\eta^{\varphi, \zeta}$. Recall that $u$ is monotone decreasing on $\left[0, t_{1}^{*}\right]$, monotone increasing on $\left[t_{1}^{*}, t_{2}^{*}\right]$, positive on $\left[0, t_{1}\right) \cup\left(t_{2}, t_{2}^{*}\right]$, and negative on $\left(t_{1}, t_{2}\right)$. In addition, $u(\eta(t))<0$ for all $t \in\left(t_{1}^{*}, t_{2}^{*}\right)$.

Define $\kappa_{-1}=t_{2}^{*}$ and $\kappa_{j}=\eta\left(\kappa_{j-1}\right)$ for $j \in\{0, \ldots, J\}$, where $J$ is the unique integer such that $\kappa_{J} \in\left(t_{1}, t_{1}^{*}\right]$. Let

$$
m_{j}=\max _{t \in\left[\kappa_{j}, \kappa_{j-1}\right]}|u(t)|, \quad j \in\{0, \ldots, J\} .
$$




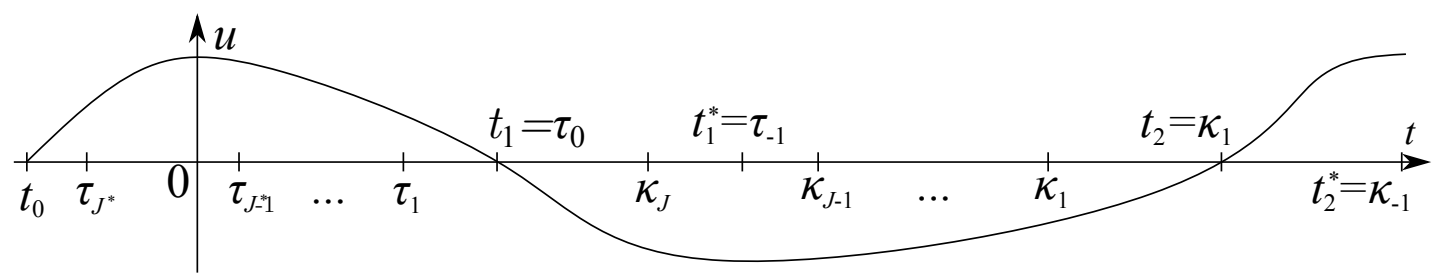

Figure 3: The sequences $\left(\kappa_{j}\right)_{j=-1}^{J}$ and $\left(\tau_{j}\right)_{j=-1}^{J^{*}}$

Observe that, by $v(t)=v^{\varphi, \zeta}(t) \in[A, B], t \geq 0$, and inequality (4.5), we have $m_{j} \leq$ $e^{f_{1}\left(T_{2}+r\right)} \max \{-A, B\}$, and hence $m_{j} \leq L_{1}, j \in\{0, \ldots, J\}$. By $C(t) \in\left[c_{0}, c_{1}\right], t \geq 0$, and equation (4.4),

$$
\begin{aligned}
m_{j} & \geq u\left(\kappa_{j-1}\right)-u\left(\kappa_{j}\right)=\int_{\kappa_{j}}^{\kappa_{j-1}} \dot{u}(t) d t=-\int_{\kappa_{j}}^{\kappa_{j-1}} C(t) u(\eta(t)) d t \\
& \geq-c_{0} \int_{\kappa_{j}}^{\kappa_{j-1}} u(\eta(t)) d t=-c_{0} \int_{\kappa_{j+1}}^{\kappa_{j}} u(t) d \eta^{-1}(t) \geq-c_{0} \frac{a}{c} \int_{\kappa_{j+1}}^{\kappa_{j}} u(t) d t
\end{aligned}
$$

for $j \in\{0, \ldots, J-1\}$. The last integral can be estimated with the area of a rectangular triangle with height $m_{j+1}$ and slope $L_{1}$ since $m_{j+1} / L_{1}<1 \leq \kappa_{j}-\kappa_{j+1}$. So, for $j \in$ $\{0, \ldots, J-1\}$, we have

$$
m_{j} \geq c_{0} \frac{a}{c} \int_{\kappa_{j+1}}^{\kappa_{j}}|u(t)| d t \geq c_{0} \frac{a}{c} \frac{m_{j+1}^{2}}{2 L_{1}}=\frac{m_{j+1}^{2}}{\beta} .
$$

As $u$ is decreasing on $\left[t_{1}, t_{1}^{*}\right]$, increasing on $\left[t_{1}^{*}, t_{2}^{*}\right]$, and $\beta>1$, by induction, we have

$$
\left|u\left(t_{2}^{*}\right)\right|=m_{0} \geq \frac{m_{J}^{2^{J}}}{\beta^{1+2+\ldots+2^{J-1}}} \geq\left(\frac{m_{J}}{\beta}\right)^{2^{J}}=\left(\frac{\left|u\left(t_{1}^{*}\right)\right|}{\beta}\right)^{2^{J}} .
$$

Similarly, define $\tau_{-1}=t_{1}^{*}$ and $\tau_{j}=\eta\left(\tau_{j-1}\right)$ for $j \in\left\{0, \ldots, J^{*}\right\}$, where $J^{*}$ is the unique integer such that $\tau_{J^{*}} \in\left(t_{0}, 0\right]$. Let

$$
\mu_{j}=\max _{t \in\left[\tau_{j}, \tau_{j-1}\right]}|u(t)|, \quad j \in\left\{0, \ldots, J^{*}\right\} .
$$

Analogously to the above estimations, we have

$$
\left|u\left(t_{1}^{*}\right)\right|=\mu_{0} \geq \frac{\mu_{J^{*}}^{J^{*}}}{\beta^{1+2+\ldots+2^{J^{*}-1}}} \geq\left(\frac{\mu_{J}}{\beta}\right)^{2^{J^{*}}} \geq\left(\frac{|u(0)|}{\beta}\right)^{2^{J^{*}}} .
$$

From $\eta(t) \leq t-1$, we have $J+J^{*} \leq T_{2}+r$, and $|u(0)| \leq \beta$ by the choice of $\beta$. Combining (4.10) and (4.11),

$$
\left|u\left(t_{2}^{*}\right)\right| \geq\left(\frac{\left|u\left(t_{1}^{*}\right)\right|}{\beta}\right)^{2^{J}} \geq \frac{1}{\beta^{2^{J}}}\left(\frac{|u(0)|}{\beta}\right)^{2^{J+J^{*}}} \geq \frac{1}{\beta^{2^{T_{2}+r}}}\left(\frac{|u(0)|}{\beta}\right)^{2^{T_{2}+r}}=\frac{|u(0)|^{2^{T_{2}+r}}}{\beta^{2 \cdot 2^{T_{2}+r}}}
$$

comes. Using $|u(0)|=\varphi(0)$ and $v\left(t_{2}^{*}\right) \leq u\left(t_{2}^{*}\right)$, our statement follows.

Define

$$
\delta_{0}=\frac{d}{2 M} d e^{-\left(T_{2}+r\right)(1+L)} e^{-f_{1} r} .
$$


Proposition 4.9. If $(\varphi, \zeta) \in \mathcal{X} \times Z$ with $\|\varphi\|_{[-r, 0]} \leq \delta_{0} e^{f_{1} r}$ then $|v(t)| \leq d / 2$ for all $t \in\left[-r, T_{2}+r\right]$.

Proof. Observe that $\Theta(t, 0, \zeta)=\left(0, z^{0, \zeta}(t)\right), t \geq 0, \zeta \in Z$. Let $(\varphi, \zeta) \in \mathcal{X} \times Z$, and $v=v^{\varphi, \zeta}, z=z^{\varphi, \zeta}$. Proposition 4.1 gives

$$
\begin{aligned}
\left\|v_{t}\right\|_{[-r, 0]} & \leq\left\|v_{t}\right\|_{[-r, 0]}+\left|z(t)-z^{0, \zeta}(t)\right|=\left\|\left(v_{t}, z(t)\right)-\left(0, z^{0, \zeta}(t)\right)\right\|_{C_{[-r, 0]} \times \mathbb{R}} \\
& =\|\Theta(t, \varphi, \zeta)-\Theta(t, 0, \zeta)\|_{C_{[-r, 0]} \times \mathbb{R}} \leq M\|\varphi\|_{[-r, 0]} e^{t(1+L)} \\
& \leq M\|\varphi\|_{[-r, 0]} e^{\left(T_{2}+r\right)(1+L)} \leq \frac{d}{2}
\end{aligned}
$$

provided $t \in\left[0, T_{2}+r\right]$ and $\|\varphi\| \leq \delta_{0} e^{f_{1} r}$.

Proposition 4.10. If $(\varphi, \zeta) \in W$ with $\varphi(0) \leq \delta_{0}$, then $z\left(t_{2}^{*}\right) \leq[\zeta-d / c]^{+}$.

Proof. Let $(\varphi, \zeta) \in W$ with $\varphi(0) \leq \delta_{0}$, and let $v=v^{\varphi, \zeta}, z=z^{\varphi, \zeta}, y=y^{\varphi, \zeta}$.

From $(\varphi, \zeta) \in W$ it follows that $0 \leq \varphi(s) \leq \varphi(0) e^{f_{1} r} \leq \delta_{0} e^{f_{1} r}, s \in[-r, 0]$. proposition 4.9 can be applied to get $|v(t)| \leq d / 2$ for all $t \in\left[-r, T_{2}+r\right]$.

Recall that $t_{0}=-\zeta-1, y\left(t_{0}\right)=c \zeta$, and $y$ satisfies equation (1.9) a.e. in $\left[t_{0}, \infty\right)$. Moreover, $z\left(t_{2}^{*}\right)=(1 / c) y\left(t_{2}^{*}-z\left(t_{2}^{*}\right)-1\right)=(1 / c) y\left(t_{2}\right)$.

Observe that if $y(t)>0$ on an interval $I \subset\left[t_{0}, T_{2}+r\right]$ then, by $|v(t)| \leq d / 2$ on $\left[-r, T_{2}+r\right]$, we have $\dot{y}(t) \leq d / 2-d=-d / 2$ a.e. in $I$. It follows that either $y\left(t_{2}\right)=0$, or $y(t)>0$ for all $t \in\left[t_{0}, t_{2}\right]$. In case $y\left(t_{2}\right)=0$ the statement trivially holds since $z\left(t_{2}^{*}\right)=(1 / c) y\left(t_{2}\right)=0$. Assume that $y(t)>0$ for all $t \in\left[t_{0}, t_{2}\right]$. Then $z\left(t_{2}^{*}\right)>0$. By inequality (4.5), $t_{2}-t_{0} \geq 2$, we find

$$
0<z\left(t_{2}^{*}\right)=\frac{1}{c} y\left(t_{2}\right)=\frac{1}{c}\left(y\left(t_{0}\right)+\int_{t_{0}}^{t_{2}} \dot{y}(t) d t\right) \leq \frac{1}{c}\left(c \zeta-\frac{d}{2}\left(t_{2}-t_{0}\right)\right) \leq \zeta-\frac{d}{c}
$$

In this case, $\zeta$ has to be greater than $d / c$.

Therefore, either $\zeta \in[0, d / c]$ and $z\left(t_{2}^{*}\right)=0$, or $\zeta>d / c$ and $z\left(t_{2}^{*}\right) \leq \zeta-d / c$.

We need a function $\alpha \in C^{2}([0, q / c], \mathbb{R})$ with the properties

$(\alpha 1) \alpha(0)=0$,

$(\alpha 2) \alpha^{\prime}(\xi)>0, \alpha^{\prime \prime}(\xi)>0$ for all $\xi \in(0, q / c]$,

$(\alpha 3) \alpha(q / c) \leq \theta\left(\delta_{0}\right)^{\rho}$

moreover, in case $d<q$,

$(\alpha 4) \alpha(\xi-(d / c)) \leq \theta(\alpha(\xi))^{\rho}$ for all $\xi \in[d / c, q / c]$.

Proposition 4.11. There exists $\alpha \in C^{2}([0, q / c], \mathbb{R})$ such that $(\alpha 1)-\left(\alpha_{4}\right)$ are satisfied.

Proof. If $d \geq q$, we only need $(\alpha 1)-(\alpha 3)$ to hold, and it is easy to find a function $\alpha$ satisfying them.

Assume that $d<q$. We look for $\alpha$ in the form

$$
\alpha(\xi)=a_{1} \exp \left(-a_{2} \exp \left(\frac{a_{3}}{\xi}\right)\right) \quad \text { for } \xi \in\left(0, \frac{q}{c}\right]
$$


with some $a_{1}>0, a_{2}>0, a_{3}>0$ determined later. For $\xi \in(0, q / c]$, we have

$$
\begin{aligned}
\alpha^{\prime}(\xi) & =\frac{a_{1} a_{2} a_{3}}{\xi^{2}} \exp \left(\frac{a_{3}}{\xi}-a_{2} \exp \left(\frac{a_{3}}{\xi}\right)\right), \\
\alpha^{\prime \prime}(\xi) & =\frac{a_{1} a_{2} a_{3}}{\xi^{4}}\left(a_{2} a_{3} \exp \left(\frac{a_{3}}{\xi}\right)-a_{3}-2 \xi\right) \exp \left(\frac{a_{3}}{\xi}-a_{2} \exp \left(\frac{a_{3}}{\xi}\right)\right) .
\end{aligned}
$$

It is elementary to see that

$$
\alpha(\xi) \rightarrow 0, \alpha^{\prime}(\xi) \rightarrow 0, \alpha^{\prime \prime}(\xi) \rightarrow 0 \quad \text { as } \xi \rightarrow 0^{+} .
$$

Then, by setting $\alpha(0)=0$, it follows that $\alpha \in C^{2}([0, q / c], \mathbb{R})$. Condition $(\alpha 1)$ holds by definition. The property for $\alpha^{\prime}$ in $(\alpha 2)$ is obvious from the above form of $\alpha^{\prime}(\xi)$. From the above expression for $\alpha^{\prime \prime}(\xi)$ it is clear that $\alpha^{\prime \prime}(\xi)>0$ for all $\xi \in(0, q / c]$ if

$$
a_{2} a_{3} \exp \left(\frac{a_{3}}{\xi}\right)>a_{3}+2 \xi \quad \text { for all } \xi \in\left(0, \frac{q}{c}\right]
$$

which is guaranteed by $a_{2} a_{3} \exp \left(a_{3} c / q\right)>a_{3}+q / c$, that is,

Property $(\alpha 3)$ holds if

$$
a_{2}>\left(1+2 \frac{q}{a_{3} c}\right) \exp \left(-\frac{a_{3} c}{q}\right) \text {. }
$$

$$
a_{1} \leq \theta \delta_{0}^{\rho} \exp \left(a_{2} \exp \left(\frac{a_{3} c}{q}\right)\right)
$$

Inequality $(\alpha 4)$ is valid if

$$
a_{1} \exp \left(-a_{2} \exp \left(\frac{a_{3}}{\xi-(d / c)}\right)\right) \leq \theta a_{1}^{\rho} \exp \left(-a_{2} \rho \exp \left(\frac{a_{3}}{\xi}\right)\right)
$$

for all $\xi \in(d / c, q / c]$. This inequality holds if both

$$
a_{1} \leq \theta a_{1}^{\rho} \quad \text { and } \quad \exp \left(\frac{a_{3}}{\xi-(d / c)}\right) \geq \rho \exp \left(\frac{a_{3}}{\xi}\right) \quad \text { for all } \xi \in\left(\frac{d}{c}, \frac{q}{c}\right]
$$

are satisfied, that is, by $\rho>1$,

$$
a_{1} \geq \theta^{\frac{1}{1-\rho}}
$$

and

$$
a_{3} \geq \xi\left(\frac{c}{d} \xi-1\right) \log \rho \quad \text { for all } \xi \in\left(\frac{d}{c}, \frac{q}{c}\right] .
$$

Since $\xi \mapsto \xi((c / d) \xi-1) \log \rho$ is increasing on $(d / c, q / c]$, the last inequality is guaranteed by

$$
a_{3} \geq \frac{q}{c}\left(\frac{q}{d}-1\right) \log \rho
$$

We have to find $a_{1}>0, a_{2}>0, a_{3}>0$ so that all Inequalities (4.12), (4.13), (4.14) and (4.15) are true.

First, fix $a_{1}>0$ so that (4.14) is satisfied. Now, choose $a_{3}^{*}>0$ such that (4.15) holds for all $a_{3} \geq a_{3}^{*}$. In the next step, using that the expression on the right hand side of (4.12) is monotone decreasing in $a_{3}$, we can fix $a_{2}>0$ such that (4.12) is valid for all $a_{3} \geq a_{3}^{*}$. Finally, as $a_{1}$ and $a_{2}$ are fixed, one can find a sufficiently large $a_{3} \geq a_{3}^{*}$ so that (4.13) holds as well. This completes the proof. 
With the $\alpha$ given in proposition 4.11 , recall that $K_{0}=\left(f_{1}+g_{1}\right) \max \{-A, B\}, K_{1}=$ $r K_{0}$, and the sets $W_{\alpha, K_{0}}, W_{\alpha, K_{1}}, V_{\alpha, K_{1}}$ are defined by Formulas (1.11), (1.12).

Proposition 4.12. The set $V_{\alpha, K_{1}}$ is a compact and convex subset of $C_{[-1,0]} \times \mathbb{R}$.

Proof. Compactness of $V_{\alpha, K_{1}}$ follows in a straighforward way from the definition of $V_{\alpha, K_{1}}$ and from the Arzela-Ascoli theorem.

In order to show the convexity of $V_{\alpha, K_{1}}$, let $\left(\psi^{1}, \zeta^{1}\right)$ and $\left(\psi^{2}, \zeta^{2}\right)$ be in $V_{\alpha, K_{1}}$, and set $(\psi, \zeta)=\lambda\left(\psi^{1}, \zeta^{1}\right)+(1-\lambda)\left(\psi^{2}, \zeta^{2}\right)$ with some $\lambda \in[0,1]$. proposition 4.11 guarantees the convexity of $\alpha$. Hence

$$
\begin{aligned}
\psi(0) & =\lambda \psi^{1}(0)+(1-\lambda) \psi^{2}(0) \geq \lambda \alpha\left(\zeta^{1}\right)+(1-\lambda) \alpha\left(\zeta^{2}\right) \\
& \geq \alpha\left(\lambda \zeta^{1}+(1-\lambda) \zeta^{2}\right)=\alpha(\zeta) .
\end{aligned}
$$

All other properties of $V_{\alpha, K_{1}}$ are obviously preserved by the convex combination.

By definition, $W_{\alpha, K_{1}} \subset W_{0}$. Therefore, the map $P$ is defined on $W_{\alpha, K_{1}}$. We know that $W_{0}$ and $W$ are invariant under $P$. The next result shows the invariance of $W_{\alpha, K_{1}}$, and slightly more since, by $K_{0}<K_{1}, W_{\alpha, K_{0}} \subseteq W_{\alpha, K_{1}}$.

Proposition 4.13. $P\left(W_{\alpha, K_{1}}\right) \subseteq W_{\alpha, K_{0}}$.

Proof. We have $P(0,0)=(0,0) \in W_{\alpha, K_{0}}$. Suppose $(\varphi, \zeta) \in W_{\alpha, K_{1}} \backslash\{(0,0)\}$. Then the inequality $\varphi(0) \geq \alpha(\zeta)$ and the nondecreasing property of $[-r, 0] \ni s \mapsto \varphi(s) e^{f_{1} s} \in \mathbb{R}$ combined imply that $(\varphi, \zeta) \in W$. By proposition 4.7, $P(\varphi, \zeta)=\Gamma\left(\Theta\left(t_{2}^{*}, \varphi, \zeta\right)\right) \in W$. Thus, two facts remain to show: $\operatorname{lip}\left(\widehat{v_{t_{2}^{*}}}\right) \leq K_{0}$, and that $P$ preserves the property $\varphi(0) \geq \alpha(\zeta)$, i.e., $v\left(t_{2}^{*}\right) \geq \alpha\left(z\left(t_{2}^{*}\right)\right)$.

From equation (4.3) and from $v_{t} \in \mathcal{X}$ it follows that $|\dot{v}(t)| \leq K_{0}$ for all $t>0$. Hence the definition of $\widehat{v_{t_{2}^{*}}}$ and $0<t_{2}<t_{2}^{*} \operatorname{imply} \operatorname{lip}\left(\widehat{v_{t_{2}^{*}}}\right) \leq K_{0}$.

By $(\varphi, \zeta) \in W_{\alpha, K_{1}} \backslash\{(0,0)\} \subset W$ we have $\varphi(0) \geq \alpha(\zeta)$, and want to prove $v\left(t_{2}^{*}\right) \geq$ $\alpha\left(z\left(t_{2}^{*}\right)\right)$. There are two cases.

Case 1. $\varphi(0) \geq \delta_{0}$. Then, by proposition 4.8, properties $(\alpha 2),(\alpha 3)$ of $\alpha$, and $z\left(t_{2}^{*}\right) \in$ $[0, q / c]$, one obtains

$$
v\left(t_{2}^{*}\right) \geq \theta(\varphi(0))^{\rho} \geq \theta\left(\delta_{0}\right)^{\rho} \geq \alpha\left(\frac{q}{c}\right) \geq \alpha\left(z\left(t_{2}^{*}\right)\right) .
$$

Case 2. $\varphi(0)<\delta_{0}$. proposition 4.10 gives $z\left(t_{2}^{*}\right) \leq[\zeta-d / c]^{+}$. If $\zeta \leq d / c$ then $z\left(t_{2}^{*}\right)=0$, and, by $(\alpha 1)$, trivially $v\left(t_{2}^{*}\right) \geq 0=\alpha(0)=\alpha\left(z\left(t_{2}^{*}\right)\right)$. If $\zeta>d / c$ then applying proposition 4.8, $\varphi(0) \geq \alpha(\zeta),(\alpha 4)$ and $(\alpha 2)$, we conclude

$$
v\left(t_{2}^{*}\right) \geq \theta(\varphi(0))^{\rho} \geq \theta(\alpha(\zeta))^{\rho} \geq \alpha\left(\zeta-\frac{d}{c}\right)=\alpha\left(\left[\zeta-\frac{d}{c}\right]^{+}\right) \geq \alpha\left(z\left(t_{2}^{*}\right)\right) .
$$

This completes the proof.

Define the subsets

$$
\begin{aligned}
& H_{1}=\left\{(\psi, \zeta) \in C_{[-1,0]} \times Z \mid \psi(-1)=0\right\} \subset C_{[-1,0]} \times \mathbb{R} \\
& H_{r}=\left\{(\varphi, \zeta) \in C_{[-r, 0]} \times Z \mid \varphi(s)=0 \text { for all } s \in[-r,-\zeta-1]\right\} \subset C_{[-r, 0]} \times \mathbb{R}
\end{aligned}
$$

with the induced subspace topologies. 
Introduce the streching map $Q: H_{1} \rightarrow H_{r}$ by $Q(\psi, \zeta)=(\varphi, \zeta)$ so that

$$
\varphi(s)= \begin{cases}\psi\left(\frac{s}{\zeta+1}\right) & \text { if } s \in[-\zeta-1,0] \\ 0 & \text { if } s \in[-r,-\zeta-1]\end{cases}
$$

and the squeezing map $R: H_{r} \rightarrow H_{1}$ by $R(\varphi, \zeta)=(\psi, \zeta)$ so that

$$
\psi(s)=\varphi((\zeta+1) s) \quad \text { for all } s \in[-1,0] .
$$

Proposition 4.14. The maps $Q: H_{1} \rightarrow H_{r}, R: H_{r} \rightarrow H_{1}$ are continuous, and

$$
Q\left(V_{\alpha, K_{1}}\right) \subseteq W_{\alpha, K_{1}}, \quad R\left(W_{\alpha, K_{0}}\right) \subseteq V_{\alpha, K_{1}}
$$

Proof. In order to see the continuity of $Q$, let $(\psi, \zeta) \in H_{1}$,

$$
\left(\psi^{n}, \zeta^{n}\right)_{n=0}^{\infty} \in H_{1} \quad \text { with } \quad\left\|\left(\psi^{n}, \zeta^{n}\right)-(\psi, \zeta)\right\| \rightarrow 0 \quad \text { as } n \rightarrow \infty
$$

and let $Q(\psi, \zeta)=(\varphi, \zeta) \in H_{r}, Q\left(\psi^{n}, \zeta^{n}\right)=\left(\varphi^{n}, \zeta^{n}\right) \in H_{r}, n \in \mathbb{N}$. By definition, $\varphi(s)=\varphi^{n}(s)$ for all $s \in\left[-r,-\max \left\{\zeta, \zeta^{n}\right\}-1\right]$. For $s \in\left[-\min \left\{\zeta, \zeta^{n}\right\}-1,0\right]$ we have

$$
\begin{aligned}
\left|\varphi(s)-\varphi^{n}(s)\right| & =\left|\psi\left(\frac{s}{\zeta+1}\right)-\psi^{n}\left(\frac{s}{\zeta^{n}+1}\right)\right| \\
& \leq\left|\psi\left(\frac{s}{\zeta+1}\right)-\psi\left(\frac{s}{\zeta^{n}+1}\right)\right|+\left|\psi\left(\frac{s}{\zeta^{n}+1}\right)-\psi^{n}\left(\frac{s}{\zeta^{n}+1}\right)\right| \\
& \leq\left|\psi\left(\frac{s}{\zeta+1}\right)-\psi\left(\frac{s}{\zeta^{n}+1}\right)\right|+\left\|\psi-\psi^{n}\right\|_{[-1,0]} .
\end{aligned}
$$

If $s \in\left[-\max \left\{\zeta, \zeta^{n}\right\}-1,-\min \left\{\zeta, \zeta^{n}\right\}-1\right]$, then in case $\zeta \geq \zeta^{n}$, one can get

$$
\left|\varphi(s)-\varphi^{n}(s)\right|=\left|\psi\left(\frac{s}{\zeta+1}\right)-0\right|=\left|\psi\left(\frac{s}{\zeta+1}\right)-\psi(-1)\right|,
$$

and in case $\zeta<\zeta^{n}$, we obtain

$$
\begin{aligned}
\left|\varphi(s)-\varphi^{n}(s)\right| & =\left|0-\psi^{n}\left(\frac{s}{\zeta^{n}+1}\right)\right| \\
& \leq\left|\psi\left(\frac{s}{\zeta^{n}+1}\right)-\psi^{n}\left(\frac{s}{\zeta^{n}+1}\right)\right|+\left|\psi\left(\frac{s}{\zeta^{n}+1}\right)\right| \\
& \leq\left\|\psi-\psi^{n}\right\|_{[-1,0]}+\left|\psi\left(\frac{s}{\zeta^{n}+1}\right)-\psi(-1)\right|
\end{aligned}
$$

For fixed $(\psi, \zeta) \in H_{1}$, by using the uniform continuity of $\psi$, the above estimations yield that $\left\|(\varphi, \zeta)-\left(\varphi^{n}, \zeta^{n}\right)\right\|$ tends to zero as $n$ tends to infinity. Since the choice of the sequence $\left(\psi^{n}, \zeta^{n}\right)$ was arbitrary, this shows the continuity of $Q$ at $(\psi, \zeta) \in H_{1}$. The continuity of $R$ can be obtained analogously.

The inclusion $Q\left(V_{\alpha, K_{1}}\right) \subseteq W_{\alpha, K_{1}}$ is obvious from the definitions of $V_{\alpha, K_{1}}, W_{\alpha, K_{1}}$ and from the fact that the streching does not increase the Lipschitz constant.

Similarly, to prove the inclusion $R\left(W_{\alpha, K_{0}}\right) \subseteq V_{\alpha, K_{1}}$ we have to check how the squeezing changes the Lipschitz constant and the exponential property. From the definition of $R$ it 
is clear that the Lipschitz constant of $\psi \in C_{[-1,0]}$, given by $\psi(s)=\varphi((\zeta+1) s), s \in[-1,0]$, can be at most $\zeta+1 \leq r$ times $\operatorname{lip}(\varphi) \leq K_{0}$. The facts that

$$
[-\zeta-1,0] \ni s \mapsto \varphi(s) e^{f_{1} s} \in \mathbb{R} \text { is nondecreasing and } r \geq \zeta+1
$$

imply that the map

$$
[-1,0] \ni s \mapsto \psi(s) e^{f_{1} r s}=\varphi((\zeta+1) s) e^{f_{1}(\zeta+1) s} e^{f_{1}(r-\zeta-1) s} \text { is nondecreasing }
$$

because it is the product of two nondecreasing functions.

This completes the proof.

Now we can define the new return map

$$
\Pi: V_{\alpha, K_{1}} \in(\psi, \zeta) \mapsto R \circ P \circ Q(\psi, \zeta) \in V_{\alpha, K_{1}}
$$

In order to get the ejectivity of the fixed point $(0,0)$ of $\Pi$, we prove the following proposition.

Proposition 4.15. There exists a constant $\gamma_{1}>0$ with

$$
\sup _{t \geq 0}\left\|v_{t}^{\varphi, \zeta}\right\|_{[-r, 0]}>\gamma_{1} \quad \text { for all }(\varphi, \zeta) \in W .
$$

Proof. 1. Recall that if $(\varphi, \zeta) \in W$ then the first two zeros $t_{1}(\varphi, \zeta), t_{2}(\varphi, \zeta)$ of $v=v^{\varphi, \zeta}$ and $t_{2}^{*}=t_{2}^{*}(\varphi, \zeta)=\eta^{-1}\left(t_{2}(\varphi, \zeta)\right)$ determine the return map $P$ by

$$
P(\varphi, \zeta)=\Gamma\left(\Theta\left(t_{2}^{*}, \varphi, \zeta\right)\right)=\left(\widehat{v_{t_{2}^{*}}}, z\left(t_{2}^{*}\right)\right) \in W
$$

where $\widehat{v_{t_{2}^{*}}}(s)=v_{t_{2}^{*}}(s)$ for $s \in\left[-z\left(t_{2}^{*}\right)-1,0\right]$, and $\widehat{v_{t_{2}^{*}}}(s)=0$ for $s \in\left[-r,-z\left(t_{2}^{*}\right)-1\right]$. Set $\left(\widetilde{v}_{t}, \widetilde{z}(t)\right)=\Theta(t, P(\varphi, \zeta)), t \geq 0$. Proposition 4.1 implies that

$$
\widetilde{v}(t)=v\left(t_{2}^{*}+t\right)\left(t \geq-z\left(t_{2}^{*}\right)-1\right) \quad \text { and } \quad \widetilde{z}(t)=z\left(t_{2}^{*}+t\right)(t \geq 0) .
$$

Replacing $(\varphi, \zeta) \in W$ with $P(\varphi, \zeta) \in W$, using the Equalities (4.17) and induction, it can be shown that the zeros of $v^{\varphi, \zeta}$ in $[0, \infty)$ form an increasing sequence $\left(s_{k}\right)_{k=1}^{\infty}$ with $s_{k+1}>s_{k}+1$, and the iterates $P^{k}(\varphi, \zeta)$ are given by

$$
P^{k}(\varphi, \zeta)=\Gamma\left(\Theta\left(s_{2 k}^{*}, \varphi, \zeta\right)\right)=\left(\widehat{v_{s_{2 k}^{*}}}, z\left(s_{2 k}^{*}\right)\right) \in W
$$

where $s_{2 k}^{*}=\eta^{-1}\left(s_{2 k}\right), k \in \mathbb{N}$. Moreover, for the solution $\left(\widetilde{v}_{t}^{k}, \widetilde{z}^{k}(t)\right)=\Theta\left(t, P^{k}(\varphi, \zeta)\right)$, $t \geq 0$, Relations (4.17) hold with $\widetilde{v}^{k}, \widetilde{z}^{k}, s_{2 k}^{*}$ instead of $\widetilde{v}, \widetilde{z}, t_{2}^{*}$.

2. Choose $k_{0} \in \mathbb{N}$ so that $2 k_{0}>T_{0}$. We claim that if $(\varphi, \zeta) \in W$ is given with $\sup _{t \geq 0}\left\|v_{t}^{\varphi, \zeta}\right\| \leq d / 2$, then the solution $(\bar{v}, \bar{z})$, with initial condition $\left(\bar{v}_{0}, \bar{z}(0)\right)=P^{k_{0}}(\varphi, \zeta) \in$ $W$, satisfies

$$
\bar{z}(t)=0 \text { for all } t \geq 0, \quad \sup _{t \geq 0}\left\|\bar{v}_{t}\right\|_{[-r, 0]} \leq \sup _{t \geq 0}\left\|v_{t}^{\varphi, \zeta}\right\|_{[-r, 0]} .
$$

Let $(\varphi, \zeta) \in W$ be given with $\sup _{t \geq 0}\left\|v_{t}^{\varphi, \zeta}\right\|_{[-r, 0]} \leq d / 2$. By proposition 4.2 with $\tau_{1}=-\zeta-1$ and $\tau_{2}$ arbitrarily large, $z^{\varphi, \zeta}(t)=0$ for all $t \geq T_{0}$. By Step 1 ,

$$
\bar{v}(t)=v^{\varphi, \zeta}\left(s_{2 k_{0}^{*}}+t\right)\left(t \geq-z^{\varphi, \zeta}\left(s_{2 k}^{*}\right)-1\right), \quad \bar{z}(t)=z\left(s_{2 k_{0}^{*}}+t\right) \quad(t \geq 0) .
$$

As $s_{2 k_{0}}^{*} \geq s_{2 k_{0}}+1 \geq s_{1}+2 k_{0}-1+1>2 k_{0}>T_{0}$, the claim follows. 
3. Suppose that there is no $\gamma_{1}$ with inequality (4.16). Then there exists a sequence $\left(\varphi^{n}, \zeta^{n}\right)_{n=1}^{\infty}$ in $W$ such that

$$
\sup _{t \geq 0}\left\|v_{t}^{\varphi^{n}, \zeta^{n}}\right\|_{[-r, 0]} \leq \min \left\{\frac{d}{2}, \frac{1}{n}\right\}
$$

By Step 2, replacing $\left(\varphi^{n}, \zeta^{n}\right)$ with $P^{k_{0}}\left(\varphi^{n}, \zeta^{n}\right)$, we can assume without loss of generality that $z^{\varphi^{n}, \zeta^{n}}(t)=0$ for all $t \geq 0, n \in \mathbb{N}$. Setting $v^{n}=v^{\varphi^{n}, \zeta^{n}}, n \in \mathbb{N}$, we have

$$
\dot{v}^{n}(t)=-f\left(v^{n}(t)\right)-g\left(v^{n}(t-1)\right) \quad(t>0) .
$$

Then, for each $n \in \mathbb{N}$, the construction of the iterates $P^{j}\left(\varphi^{n}, \zeta^{n}\right), j \in \mathbb{N}$, gives a sequence $\left(t_{k}^{n}\right)_{k=0}^{\infty}$ such that

$$
t_{0}^{n}=-1, t_{k}^{n}+1<t_{k+1}^{n}, v^{n}\left(t_{k}^{n}\right)=0,\left\{\begin{array}{ll}
v^{n}(t)>0 & \text { for } t \in\left(t_{2 k}^{n}, t_{2 k+1}^{n}\right), \\
v^{n}(t)<0 & \text { for } t \in\left(t_{2 k+1}^{n}, t_{2 k+2}^{n}\right)
\end{array} .\right.
$$

for all integers $k \geq 0$.

We claim that

$$
\left\|v_{t_{k}^{n}+1}^{n}\right\|_{[-1,0]} \leq e^{f_{1}}\left|v^{n}\left(t_{k}^{n}+1\right)\right| \quad \text { for all } k \in \mathbb{N} \text {. }
$$

Recalling functions $\widetilde{f}, \widetilde{g}$, equation (4.18) can be written in the form (4.3) with $z(t)=0$. Then, for $k \in \mathbb{N}$, by using (4.19), we obtain

$$
\begin{aligned}
& \frac{d}{d s}\left[v^{n}\left(t_{2 k}^{n}+s\right) e^{f_{1} s}\right]=\dot{v}^{n}\left(t_{2 k}^{n}+s\right) e^{f_{1} s}+v^{n}\left(t_{2 k}^{n}+s\right) f_{1} e^{f_{1} s} \\
& =\left[\left(f_{1}-\widetilde{f}\left(v^{n}\left(t_{2 k}^{n}+s\right)\right)\right) v^{n}\left(t_{2 k}^{n}+s\right)-\widetilde{g}\left(v^{n}\left(t_{2 k}^{n}+s-1\right)\right) v^{n}\left(t_{2 k}^{n}+s-1\right)\right] e^{f_{1} s} \geq 0
\end{aligned}
$$

for all $s \in[0,1]$ because $v^{n}\left(t_{2 k}^{n}+s\right) \geq 0,0 \leq \widetilde{f}\left(v^{n}\left(t_{2 k}^{n}+s\right)\right) \leq f_{1}, \widetilde{g}\left(v^{n}\left(t_{2 k}^{n}+s-1\right)\right)>0$ and $v^{n}\left(t_{2 k}^{n}+s-1\right) \leq 0$. Thus,

$$
0 \leq v^{n}\left(t_{2 k}^{n}+s\right) \leq v^{n}\left(t_{2 k}^{n}+1\right) e^{f_{1}(1-s)} \leq v^{n}\left(t_{2 k}^{n}+1\right) e^{f_{1}} \quad(s \in[0,1]) .
$$

Analogously, for each $k \in \mathbb{N}$,

$$
0 \geq v^{n}\left(t_{2 k+1}^{n}+s\right) \geq v^{n}\left(t_{2 k+1}^{n}+1\right) e^{f_{1}(1-s)} \geq v^{n}\left(t_{2 k+1}^{n}+1\right) e^{f_{1}} \quad(s \in[0,1]) .
$$

This proves the claim.

By (S2), (4.18) and (4.19), we find that $t \mapsto\left|v^{n}(t)\right|$ is a decreasing function on $\left[t_{k}^{n}+\right.$ $\left.1, t_{k+1}^{n}\right]$ for all $k \in \mathbb{N}$. This fact, combined with inequality (4.20) and the choice of $\left(\varphi^{n}, \zeta^{n}\right)_{n=1}^{\infty}$, yields, for all $n \in \mathbb{N}$, the existence of an integer $k(n)>n$ such that

$$
\frac{1}{2} \sup _{s \geq 0}\left|v^{n}\left(t_{k(n)}^{n}+s\right)\right| \leq\left\|v_{t_{k(n)}^{n}+1}^{n}\right\|_{[-1,0]} \leq \frac{1}{n} .
$$

Observe that (4.19) implies $v^{n}\left(t_{k(n)}^{n}+1\right) \neq 0$. For each $n \in \mathbb{N}$, the function

$$
w^{n}:[-1, \infty) \ni t \mapsto \frac{v^{n}\left(t_{k(n)}^{n}+1+t\right)}{\left|v^{n}\left(t_{k(n)}^{n}+1\right)\right|}
$$


satisfies $\left|w^{n}(0)\right|=1$, and, by inequality (4.20),

$$
\sup _{t \geq-1}\left|w^{n}(t)\right| \leq \frac{1}{\left|v^{n}\left(t_{k(n)}^{n}+1\right)\right|} \sup _{s \geq 0}\left|v^{n}\left(t_{k(n)}^{n}+s\right)\right| \leq 2 \frac{\|\left. v_{t_{k(n)}^{n}+1}^{n}\right|_{[-1,0]}}{\left|v^{n}\left(t_{k(n)}^{n}+1\right)\right|} \leq 2 e^{f_{1}} .
$$

Moreover, equation (4.18), the definitions of $\widetilde{f}, \widetilde{g}$ and $w^{n}$ imply

$$
\dot{w}^{n}(t)=-\widetilde{f}\left(v^{n}\left(t_{k(n)}^{n}+1+t\right)\right) w^{n}(t)-\widetilde{g}\left(v^{n}\left(t_{k(n)}^{n}+t\right)\right) w^{n}(t-1)
$$

for all $t>0$. Hence $\left|\dot{w}^{n}(t)\right| \leq 2\left(f_{1}+g_{1}\right) e^{f_{1}}$ for all $t>0$.

We can apply the Arzela-Ascoli theorem and the Cantor diagonalization process for the sequence $\left(\left.w^{n}\right|_{[0, \infty)}\right)_{n=1}^{\infty}$ of continuous functions to find a subsequence $\left(n_{l}\right)_{l=1}^{\infty}$ of $\mathbb{N}$ and a continuous function $w:[0, \infty) \rightarrow \mathbb{R}$ so that

$$
w^{n_{l}}(t) \rightarrow w(t) \text { as } l \rightarrow \infty \text { uniformly in } t \text { on compact subsets of }[0, \infty) .
$$

From (4.21) and the definitions of $\tilde{f}, \widetilde{g}$ it follows that

$$
\tilde{f}\left(v^{n_{l}}\left(t_{k\left(n_{l}\right)}^{n_{l}}+1+t\right)\right) \rightarrow f^{\prime}(0) \quad \text { and } \quad \widetilde{g}\left(v^{n_{l}}\left(t_{k\left(n_{l}\right)}^{n_{l}}+t\right)\right) \rightarrow g^{\prime}(0) \quad \text { as } l \rightarrow \infty .
$$

Hence the right-hand side of equation (4.22) converges to $-f^{\prime}(0) w(t)-g^{\prime}(0) w(t-1)$ uniformly on compact subsets of $[1, \infty)$. Consequently, $w$ is differentiable on $(1, \infty)$, and satisfies

$$
\dot{w}(t)=-f^{\prime}(0) w(t)-g^{\prime}(0) w(t-1) \quad(t>1) .
$$

So, we obtained a continuous $w:[0, \infty) \rightarrow \mathbb{R}$ so that $|w(0)|=1,|w(t)| \leq 2 e^{f_{1}}$ for all $t \geq 0$, the restriction $\left.w\right|_{(1, \infty)}$ is differentiable and equation (4.23) holds. From (4.19) observe that $w^{n}$ has at most one sign change on $[0,1], n \in \mathbb{N}$. Then $w$ can have at most one sign change on $[0,1]$ as well. By proposition 2.1 it follows that $w$ is unbounded on $[0, \infty)$. This is a contradiction, and the proof is complete.

Proposition 4.16. $(0,0) \in V_{\alpha, K_{1}}$ is an ejective fixed point of $\Pi$.

Proof. As the maps $Q$ and $R$ act on $(\psi, \zeta) \in C_{[-1,0]} \times \mathbb{R}$ and $(\varphi, \zeta) \in C_{[-r, 0]} \times \mathbb{R}$, respectively, such that the norms of $\psi$ and $\varphi$ are preserved, it suffices to show the ejectivity of the fixed pont $(0,0)$ of $P$ on $W_{\alpha, K_{1}}$.

By proposition 4.1 , and by the fact that $(0,0)$ is an equilibrium point, there exists $\gamma_{2}>0$ such that if $(\varphi, \zeta) \in W$ and $\|(\varphi, \zeta)\|=\|\varphi\|_{[-r, 0]}+\zeta<\gamma_{2}$ then

$$
\left\|\left(v_{t}^{\varphi, \zeta}, z^{\varphi, \zeta}(t)\right)\right\|=\left\|v_{t}^{\varphi, \zeta}\right\|_{[-r, 0]}+z^{\varphi, \zeta}(t)<\gamma_{1} \text { for all } t \in\left[0, T_{2}+r\right] .
$$

Suppose that there exists $(\varphi, \zeta) \in W$ so that

$$
\left\|P^{k}(\varphi, \zeta)\right\|<\gamma_{2} \quad \text { for all } k \in\{0,1,2, \ldots\} .
$$

Let $v=v^{\varphi, \zeta}, z=z^{\varphi, \zeta}$. In Step 1 of the proof of proposition 4.15 we saw that the increasing sequence $\left(s_{k}\right)_{k=1}^{\infty}$ of the zeros of $v$ in $(0, \infty)$ determines the iterates $P^{k}(\varphi, \zeta)$ by $P^{k}(\varphi, \zeta)=$ $\Gamma\left(\Theta\left(s_{2 k}^{*}, \varphi, \zeta\right)\right)=\left(\widehat{v_{s_{2}^{*}}^{*}}, z\left(s_{2 k}^{*}\right)\right)$, where $s_{2 k}^{*}=\eta^{-1}\left(s_{2 k}\right), k \in \mathbb{N}$. Moreover, $2 \leq s_{2}^{*} \leq T_{2}+r$, $2 \leq s_{2 k+2}^{*}-s_{2 k}^{*} \leq T_{2}+r, k \in \mathbb{N}$, and the solutions $\left(v_{t}^{k}, z^{k}(t)\right)=\Theta\left(t, P^{k}(\varphi, \zeta)\right)$ satisfy 
$v^{k}(t)=v\left(s_{2 k}^{*}+t\right), t \geq-z\left(s_{2 k}^{*}\right)-s, z^{k}(t)=z\left(s_{2 k}^{*}+t\right), t \geq 0, k \geq \mathbb{N}$. Combining these facts with the choice of $\gamma_{2}$ and assumption (4.24), it can be obtained by induction that

$$
\left\|v_{t}^{\varphi, \zeta}\right\|_{[-r, 0]} \leq\left\|v_{t}^{\varphi, \zeta}\right\|_{[-r, 0]}+z^{\varphi, \zeta}(t)<\gamma_{1} \quad \text { for all } t \geq 0 .
$$

This inequality contradicts the existence of $\gamma_{1}>0$ with inequality (4.16).

Therefore, ejectivity of the trivial fixed point $(0,0)$ of $P$ on $W_{\alpha, K_{1}}$ follows with the open set $W_{\alpha, K_{1}} \cap U$, where

$$
U=\left\{(\varphi, \zeta) \in C_{[-r, 0]} \times \mathbb{R}:\|(\varphi, \zeta)\|<\gamma_{2}\right\}
$$

The proof is complete.

Now we are able to show the main result.

Theorem 4.17. Assume that Conditions (S1)-(S4) hold. Then System (1.8), (1.9), (1.10) has a slowly oscillatory periodic solution.

Proof. By proposition 4.12 the set $V_{\alpha, K_{1}}$ is a compact and convex subset of the Banach space $C_{[-1,0]} \times \mathbb{R}$. Proposition ?? combined show that the map $\Pi: V_{\alpha, K_{1}} \rightarrow V_{\alpha, K_{1}}$ is continuous. According to proposition 4.16 the fixed point $(0,0)$ of $\Pi$ is ejective. Then theorem B guarantees that $\Pi$ has a nonejective fixed point $\left(\psi^{*}, \zeta^{*}\right)$ in $V_{\alpha, K_{1}}$. By the ejectivity of $(0,0)$, we have $\left(\psi^{*}, \zeta^{*}\right) \neq(0,0)$, in particular $\psi^{*} \neq 0$.

Define $\varphi^{*} \in C_{[-r, 0]}$ so that $\left(\varphi^{*}, \zeta^{*}\right)=Q\left(\psi^{*}, \zeta^{*}\right)$. Let $\left(\varphi^{* *}, \zeta^{* *}\right)=P\left(\varphi^{*}, \zeta^{*}\right)$. From $R\left(\varphi^{* *}, \zeta^{* *}\right)=\left(\psi^{*}, \zeta^{*}\right)$ one obtains $\zeta^{* *}=\zeta^{*}$. Therefore, $\varphi^{* *}(s)=0=\varphi^{*}(s)$ for all $s \in\left[-r,-\zeta^{*}-1\right]$. Moreover, $Q$ streches $\psi^{*}$ with the same factor $\zeta^{*}+1$ as $R$ squeezes $\varphi^{* *}$. Then necessarily

$$
\varphi^{*}(s)=\psi^{*}\left(\frac{s}{\zeta^{*}+1}\right)=\varphi^{* *}\left(\left(\zeta^{*}+1\right) \frac{s}{\zeta^{*}+1}\right)=\varphi^{* *}(s)
$$

for all $s \in\left[-\zeta^{*}-1,0\right]$. Therefore, $\left(\varphi^{* *}, \zeta^{* *}\right)=\left(\varphi^{*}, \zeta^{*}\right)$, that is, $\left(\varphi^{*}, \zeta^{*}\right)=Q\left(\psi^{*}, \zeta^{*}\right)$ is a nontrivial fixed point of $P$.

The solution $\left(v^{\varphi^{*}, \zeta^{*}}, z^{\varphi^{*}, \zeta^{*}}\right)$ of System (1.8), (1.9), (1.10) defines a slowly oscillatory periodic solution $(v, z): \mathbb{R} \rightarrow \mathbb{R}$ in the following way. As $\left(\varphi^{*}, \zeta^{*}\right)$ is a fixed point of $P$, the restriction $\left.v^{\varphi^{*}, \zeta^{*}}\right|_{[0, \infty)}$ of $v^{\varphi^{*}, \zeta^{*}}$ and $z^{\varphi^{*}, \zeta^{*}}$ are $t_{2}^{*}$-periodic functions with $t_{2}^{*}=t_{2}^{*}\left(\varphi^{*}, \zeta^{*}\right)>0$. A $t_{2}^{*}$-periodic extension of $\left.v^{\varphi^{*}, \zeta^{*}}\right|_{[0, \infty)}$ and $z^{\varphi^{*}, \zeta^{*}}$ from $[0, \infty)$ to $\mathbb{R}$ give the slowly oscillating periodic solution $(v, z): \mathbb{R} \rightarrow \mathbb{R}$.

\section{$5 \quad$ Examples}

1. Consider System (1.4), (1.2), (1.3) with $U \in C^{2}((0, \infty), \mathbb{R})$ and $p \in C^{1}((0, \infty), \mathbb{R})$ satisfying

$$
U^{\prime}(\xi)>0, U^{\prime \prime}(\xi)<0, p(\xi)>0, p^{\prime}(\xi)>0 \text { for all } \xi \geq 0 .
$$

Then $U^{\prime \prime}-p^{\prime}<0$, so $U^{\prime}-p$ has at most one zero. Assume that there exists an $x_{*}>0$ with $U^{\prime}\left(x_{*}\right)-p\left(x_{*}\right)=0$.

For fixed constants $\kappa, a, b, c, q, r_{0}, r_{1}$ with $\kappa>0,0<a<x_{*}<c<b, q>0, r_{0} \geq 0$, $r_{1}>0$ set $K=\kappa\left[\max _{\xi \in[a, b]} \xi U^{\prime}(\xi)+b p(b)\right]$. Define $X, Y, Z$ as in section 1 and $G: X \times Z \rightarrow$ 
$\mathbb{R}$ by (1.7). Then $U \in C^{2}, p \in C^{1}$ imply Hypothesis (G1). (G2) obviously holds. As $\xi \mapsto \xi p(\xi)$ is increasing on $(0, \infty)$, Hypothesis (G3) requires

$$
a U^{\prime}(a)>b p(b), \quad b U^{\prime}(b)<a p(a) .
$$

Under the above assumptions, theorem ?? yield that System (1.4), (1.2), (1.3) is well posed both in $X \times Y$ and $X \times Z$, and all solutions can be extended to the right half line.

2. Now consider System (1.4), (1.2), (1.3) when $r_{0}=0, r_{1}=1, c>1, q>0$, and $U(\xi)=-\xi^{-\alpha} / \alpha, p(\xi)=\xi^{\beta}$ with some positive $\alpha$ and $\beta$. Then $U^{\prime}(\xi)=\xi^{-\alpha-1}, \xi U^{\prime}(\xi)=$ $\xi^{-\alpha}, \xi p(\xi)=\xi^{\beta+1}$, and $x_{*}=1$. In our particular case condition (5.1) holds for some $0<a<1<c<b$ if $a^{-\alpha}>b^{\beta+1}$ and $b^{-\alpha}<a^{\beta+1}$, or equivalently $a^{\alpha} b^{\beta+1}<1<a^{\beta+1} b^{\alpha}$. This can be true only if $\beta+1<\alpha$.

In order to satisfy condition (5.1) we modify the function $U$ close to zero. For $\varepsilon \in(0,1)$ define

$$
U_{\varepsilon}(\xi)=-\frac{1}{\alpha \xi^{\alpha}}-V_{\varepsilon}(\xi), \quad \text { where } \quad V_{\varepsilon}(\xi)= \begin{cases}\exp \left(\frac{1}{\xi}+\frac{1}{\xi-\varepsilon}\right) & \text { if } 0<\xi<\varepsilon \\ 0 & \text { if } \xi \geq \varepsilon\end{cases}
$$

Cleary, $V_{\varepsilon}$ and $U_{\epsilon}$ are in $C^{\infty}((0, \infty), \mathbb{R})$, and $\xi U_{\varepsilon}^{\prime}(\xi)=\xi^{-\alpha}+\left[\xi^{-1}+\xi /(\xi-\varepsilon)^{2}\right] V_{\varepsilon}(\xi)$ for all $\xi>0$. We want to find $a, b$ such that $0<a<1<c<b$, and $a U_{\varepsilon}^{\prime}(a)>b^{\beta+1}$ and $b^{-\alpha}<a^{\beta+1}$. For given $a>0$ choose $b>0$ such that $b^{-\alpha}=a^{\beta+1} / 2$, i.e., $b=2^{1 / \alpha} a^{-(\beta+1) / \alpha}$. Then $b^{-\alpha}<a^{\beta+1}$ holds. Inequality $a U_{\varepsilon}^{\prime}(a)>b^{\beta+1}$ is satisfied if

$$
a U_{\varepsilon}^{\prime}(a)>2^{\frac{\beta+1}{\alpha}} a^{-\frac{(\beta+1)^{2}}{\alpha}},
$$

which is valid if $a>0$ is small enough, since $a U_{\varepsilon}^{\prime}(a) \rightarrow \infty$ faster than $a^{-(\beta+1)^{2} / \alpha}$ as $a \rightarrow 0^{+}$. Consequently, for each fixed $\varepsilon \in(0,1)$, there exists $a=a_{\varepsilon} \in(0, \varepsilon)$ so that, by choosing $a \in\left(0, a_{\varepsilon}\right)$ and $b=2^{1 / \alpha} a^{-(\beta+1) / \alpha}$, condition (5.1) is valid with $U_{\varepsilon}$ instead of $U$. Clearly, $b \rightarrow \infty$ as $a \rightarrow 0^{+}$. In particular, we may achieve $b>c$.

Therefore, for each fixed $\varepsilon \in(0,1)$ and sufficiently small $a \in(0, \varepsilon)$, theorem 3.10 is applicable for System (1.4), (1.2), (1.3) with $r_{0}=0, r_{1}=1, p(\xi)=\xi^{\beta}$ and $U_{\varepsilon}$ instead of $U$. For the new variable $v=x-1$ we obtain System (1.8), (1.9), (1.10) with $f(v)=$ $-\kappa\left[(v+1) U_{\varepsilon}^{\prime}(v+1)-U^{\prime}(1)\right], g(v)=\kappa\left[(v+1)^{\beta+1}-1\right]$, and $d=c-1>0$. It is clear that Conditions (S1)-(S3) hold with $A=a-1, B=b-1$. We have $f^{\prime}(0)=\kappa \alpha$ and $g^{\prime}(0)=\kappa(\beta+1)$.

If $\alpha>\beta+1$ then (S5) holds. Indeed, let $\lambda \in \mathbb{C}$ with $\operatorname{Re} \lambda \geq 0$, and suppose $\lambda+\kappa \alpha+\kappa(\beta+1) e^{-\lambda}=0$. Then $\kappa \alpha \leq|\lambda+\kappa \alpha|=\left|\kappa(\beta+1) e^{-\lambda}\right| \leq \kappa(\beta+1)$, a contradiction to $\alpha>\beta+1$. Therefore, by theorem 4.3, the solution $(0,0)$ of System (1.8), (1.9), (1.10), or equivalently, the solution $(1,0)$ of $(1.4),(1.2),(1.3)$ is locally asymptotically stable.

Assume $\alpha<\beta+1$. Then there exists $\vartheta_{0} \in(\pi / 2, \pi)$ so that $-\cos \vartheta_{0}=\alpha /(\beta+1)$. Define $\kappa_{0}=-(1 / \alpha) \vartheta_{0} \cot \vartheta_{0}$. For each $\kappa>\kappa_{0}$ there exists $\vartheta_{1} \in\left(\vartheta_{0}, \pi\right)$ such that $\kappa \alpha=-\vartheta_{1} \cot \vartheta_{1}$ since $[\pi / 2, \pi) \ni \vartheta \mapsto-\vartheta \cot \vartheta \in \mathbb{R}$ increases from 0 to $\infty$. Then

$$
\kappa(\beta+1)=\frac{\beta+1}{\alpha} \kappa \alpha=-\frac{1}{\cos \vartheta_{0}}\left(-\vartheta_{1} \cot \vartheta_{1}\right)=\frac{-\cos \vartheta_{1}}{-\cos \vartheta_{0}} \frac{\vartheta_{1}}{\sin \vartheta_{1}}>\frac{\vartheta_{1}}{\sin \vartheta_{1}},
$$

and condition (2.3) is satisfied yielding (S4) for all $\kappa>\kappa_{0}$. Thus, theorem 4.17 implies, with the above particular choice of $f, g$, that System (1.8), (1.9), (1.10) has a slowly oscillatory periodic solution provided $\kappa>\kappa_{0}$ and $\alpha<\beta+1$. Equivalently, if $\alpha<\beta+1$ and $\kappa>\kappa_{0}$ then System (1.4), (1.2), (1.3) with $r_{0}=0, r_{1}=1, p(\xi)=\xi^{\beta}$ and $U_{\varepsilon}$ instead 
of $U$ has a periodic solution $(x, z)$ oscillating slowly around $x_{*}=1$. For this periodic solution $x$, we claim that

$$
x(t) \in\left[(1+\kappa r)^{-\frac{\beta+1}{\alpha}}, 1+\kappa r\right] \text { for all } t \in \mathbb{R} .
$$

Let $t_{1} \geq 0$ be such that $x\left(t_{1}\right)>1$ and $x$ has a local maximum at $t_{1}$. Then $\dot{x}\left(t_{1}\right)=0$. If $x(t)>1$ for all $t \in\left[t_{1}-r, t_{1}\right]$ then, by $x\left(t_{1}\right) U_{\varepsilon}^{\prime}\left(x\left(t_{1}\right)\right)<1$ and $x\left(t_{1}-z\left(t_{1}\right)-1\right)>1$, one obtains

$$
\dot{x}\left(t_{1}\right)=\kappa\left[x\left(t_{1}\right) U_{\varepsilon}^{\prime}\left(x\left(t_{1}\right)\right)-\left[x\left(t_{1}-z\left(t_{1}\right)-1\right)\right]^{\beta+1}\right]<0,
$$

a contradiction. Therefore, there is a maximal $t_{0} \in\left[t_{1}-r, t_{1}\right)$ such that $x\left(t_{0}\right)=1$. An integration gives

$$
x\left(t_{1}\right)=1+\int_{t_{0}}^{t_{1}} \kappa\left[x(t) U_{\varepsilon}^{\prime}(x(t))-\left[x\left(t-z\left(t_{1}\right)-1\right)\right]^{\beta+1}\right] d t \leq 1+\kappa r,
$$

and, since $t_{1}$ was an arbitrary local maximum, we obtain the upper bound in (5.2). If $t_{2} \in \mathbb{R}$ is such that $x\left(t_{2}\right)<1$ and $x$ has a local minimum at $t_{2}$, then $\dot{x}\left(t_{2}\right)=0$ and $x\left(t_{2}\right) U_{\varepsilon}^{\prime}\left(x\left(t_{2}\right)\right)=\left[x\left(t_{2}-z\left(t_{2}\right)-1\right)\right]^{\beta+1}$. Hence, using $U_{\varepsilon}^{\prime} \geq U^{\prime}$ and $x(t) \leq 1+\kappa r$ for $t \in \mathbb{R}$, the inequality

$$
\left[x\left(t_{2}\right)\right]^{-\alpha}=x\left(t_{2}\right) U^{\prime}\left(x\left(t_{2}\right)\right) \leq x\left(t_{2}\right) U_{\varepsilon}^{\prime}\left(x\left(t_{2}\right)\right) \leq[1+\kappa r]^{\beta+1}
$$

follows, yielding the lower bound in (5.2), because $t_{2}$ was an arbitrary local minimum.

Consequently, if, for a fixed $\kappa>0$, we choose $\varepsilon>0$ so that $\varepsilon<(1+\kappa r)^{-(\beta+1) / \alpha}$, $a \in(0, \varepsilon), b>\max \{c, 1+\kappa r\}$, and condition (5.1) is satisfied, then all possible periodic solutions (oscillating around $x_{*}=1$ ) of System (1.4), (1.2), (1.3), with $r_{0}=0, r_{1}=1$, $p(\xi)=\xi^{\beta}$ and $U_{\varepsilon}$ instead of $U$ have ranges in $\left[(1+\kappa r)^{-(\beta+1) / \alpha}, 1+\kappa r\right] \subset(\varepsilon, b)$, where $U_{\varepsilon}=U$. In particular, the periodic solution obtained for the modified equation (i.e. with $U_{\varepsilon}$ instead of $U$ ), is the solution of the original System (1.4), (1.2), (1.3) as well.
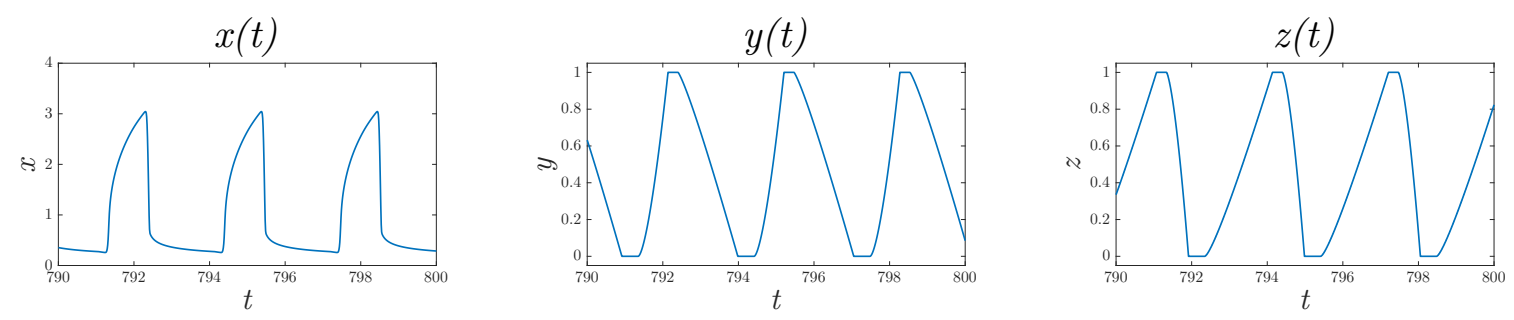

Figure 4: A numerical solution showing periodicity with $\alpha=3, \beta=1, \kappa=10, q=c=$ $1.01, x_{*}=1, r_{0}=0, r_{1}=1$.

\section{Acknowledgements}

This research was supported by the Hungarian Scientific Research Fund, Grant No. K 129322, the EU-funded Hungarian grant EFOP-3.6.2-16-2017-0015 and by the Ministry of Human Capacities, Hungary grant 20391-3/2018/FEKUSTRAT.

The authors thank the reviewers for their relevant comments that contributed to improve the paper. 


\section{References}

[1] T. Alpcan and T. Başar. "A Utility-Based Congestion Control Scheme for InternetStyle Networks with Delay". In: IEEE INFOCOM 2003. Twenty-second Annual Joint Conference of the IEEE Computer and Communications Societies (IEEE Cat. No.03CH37428) (Apr. 2003).

[2] O. Arino, K. P. Hadeler, and M. L. Hbid. "Existence of Periodic Solutions for Delay Differential Equations with State Dependent Delay". In: Journal of Differential Equations 144.2 (Apr. 1998), pp. 263-301.

[3] C. Briat et al. "Nonlinear State-Dependent Delay Modeling and Stability Analysis of Internet Congestion Control". In: 49th IEEE Conference on Decision and Control (CDC) (Dec. 2010).

[4] F. E. Browder. "A Further Generalization of the Schauder Fixed Point Theorem". In: Duke Mathematical Journal 32.4 (1965), pp. 575-578.

[5] N.A. Cookson et al. "Queueing up for Enzymatic Processing: Correlated Signaling Through Coupled Degradation". In: Molecular Systems Biology 7.1 (Dec. 2011).

[6] K. Deimling. Multivalued Differential Equations. Berlin: Walter de Gruyter, 1992.

[7] O. Diekmann et al. Delay Equations. New York: Springer-Verlag, 1995.

[8] M. Garavello and B. Piccoli. Traffic Flow on Networks: Conservation Laws Models. Springfield: AIMS, 2006.

[9] J. K. Hale and S. M. Verduyn Lunel. Introduction to Functional-Differential Equations. New York: Springer-Verlag, 1993.

[10] F. Hartung et al. "Functional Differential Equations with State-Dependent Delays: Theory and Applications". In: Handbook of Differential Equations: Ordinary Differential Equations 3 (2006), pp. 435-545.

[11] Q. Hu and J. Wu. "Global Hopf Bifurcation for Differential Equations with StateDependent Delay". In: Journal of Differential Equations 248.12 (June 2010), pp. 28012840 .

[12] F. Kelly. "Charging and rate control for elastic traffic". In: European Transactions on Telecommunications (Feb. 1997).

[13] F. Kelly, A. Maulloo, and D. Tan. "Rate Control for Communication Networks: Shadow Prices, Proportional Fairness and Stability". In: Journal of the Operational Research Society 49.3 (Mar. 1998), 237-252.

[14] T. Krisztin and O. Arino. "The Two-Dimensional Attractor of a Differential Equation with State-Dependent Delay". In: Journal of Dynamics and Differential Equations 13.3 (July 2001), 453-522.

[15] R. J. La and P. Ranjan. "Asymptotic Stability of a Rate Control System With Communication Delays". In: IEEE Transactions on Automatic Control 52.10 (Oct. 2007), pp. 1920-1925.

[16] R. J. La and P. Ranjan. "Convergence of Dual Algorithm with Arbitrary Communication Delays". In: SIAM Journal on Applied Mathematics 68.5 (2008), pp. 12471267. 
[17] R. J. La and P. Ranjan. "Stability of a Rate Control System with Averaged Feedback and Network Delay". In: Automatica 42.10 (2006), pp. 1817-1820.

[18] V. Lakshmikantham and S. Leela. Differential and Integral Inequalities. New York and London: Academic Press, Jan. 1969.

[19] P. Magal and O. Arino. "Existence of Periodic Solutions for a State Dependent Delay Differential Equation". In: Journal of Differential Equations 144.2 (Apr. 1998), pp. 263-301.

[20] J. Mallet-Paret and R. D. Nussbaum. "Boundary Layer Phenomena for DifferentialDelay Equations with State-Dependent Time Lags, I." In: Archive for Rational Mechanics and Analysis 120.2 (Nov. 1992), 99-146.

[21] J. Mallet-Paret, R. D. Nussbaum, and P. Paraskevopoulos. "Periodic solutions for functional-differential equations with multiple state-dependent time lags". In: Topological Methods in Nonlinear Analysis 3.1 (1994), pp. 101-162.

[22] P. Ranjan. Greed Considered Harmful: Nonlinear (in)stabilities in network resource allocation. 2009.

[23] P. Ranjan and R. J. La. "Global Stability Conditions for Rate Control With Arbitrary Communication Delays". In: IEEE/ACM Transactions on Networking 14.1 (Feb. 2006).

[24] P. Ranjan, R. J. La, and E. H. Abed. "Delay, Elasticity, and Stability Trade-Offs in Rate Control". In: Technical Report - The Center for Satellite and Hybrid Communication Networks CSHCN TR 2003-13 (Mar. 2003).

[25] P. Ranjan, R. J. La, and E. H. Abed. "Global Stability in the Presence of StateDependent Delay in Rate Control". In: Proceedings of the Conference on Information Sciences and Systems (2004), pp. 1099-1104.

[26] P. Ranjan, R. J. La, and E. H. Abed. "Global Stability with a State-Dependent Delay in Rate Control". In: Proceedings of the Conference on Time-Delay Systems (2004).

[27] R. Srikant. The Mathematics of Internet Congestion Control. New York: SpringerVerlag, 2004.

[28] J. Szarski. Differential Inequalities. Warsaw: Polish Scientific Publishers, 1965.

[29] H.-O. Walther. "A Periodic Solution of a Differential Equation with State-Dependent Delay". In: Journal of Differential Equations 244.8 (Apr. 2008), pp. 1910-1945.

[30] H.-O. Walther. "Stable Periodic Motion of a System Using Echo for Position Control". In: Journal of Dynamics and Differential Equations 15.1 (Jan. 2003), 143223.

[31] H.-O. Walther. "Stable Periodic Motion of a System With State Dependent Delay". In: Differential Integral Equations 15.8 (Nov. 2002), pp. 923-944.

[32] H.-O. Walther. The 2-Dimensional Attractor of $x^{\prime}(t)=-\mu x(t)+f(x(t-1))$. Memoirs of the American Mathematical Society, 1995.

[33] H.-O. Walther. "The Solution Manifold and $C^{1}$-Smoothness for Differential Equations with State-Dependent Delay". In: Journal of Differential Equations 195.1 (Nov. 2003), pp. 46-65. 